Wright State University

CORE Scholar

2016

\title{
Hybrid Particle Image Velocimetry with the Combination of Cross- Correlation and Optical Flow Method
}

Mark Bradley Johnson

Wright State University

Follow this and additional works at: https://corescholar.libraries.wright.edu/etd_all

Part of the Mechanical Engineering Commons

\section{Repository Citation}

Johnson, Mark Bradley, "Hybrid Particle Image Velocimetry with the Combination of Cross-Correlation and Optical Flow Method" (2016). Browse all Theses and Dissertations. 1481.

https://corescholar.libraries.wright.edu/etd_all/1481

This Thesis is brought to you for free and open access by the Theses and Dissertations at CORE Scholar. It has been accepted for inclusion in Browse all Theses and Dissertations by an authorized administrator of CORE Scholar. For more information, please contact library-corescholar@wright.edu. 


\title{
HYBRID PARTICLE IMAGE VELOCIMETRY WITH THE COMBINATION OF CROSS-CORRELATION AND OPTICAL FLOW METHOD
}

\author{
A thesis submitted in partial fulfillment of the \\ requirements for the degree of \\ Master of Science in Mechanical Engineering
}

By

MARK BRADLEY JOHNSON

B.S., Wright State University, 2015

2016

Wright State University 


\title{
WRIGHT STATE UNIVERSITY
}

\author{
GRADUATE SCHOOL
}

April 22, 2016

I HEREBY RECOMMEND THAT THE THESIS PREPARED UNDER MY SUPERVISION

BY Mark Bradley Johnson ENTITLED Hybrid Particle Image Velocimetry with the Combination of Cross-Correlation and Optical Flow Method BE ACCEPTED IN PARTIAL FULFILLMENT OF THE REQUIREMENTS FOR THE DEGREE OF Master of Science in Mechanical Engineering.

Zifeng Yang, Ph.D.

Thesis Director

George Huang, Ph.D.

Chair, Department of Mechanical \& Materials

Engineering

Committee on

Final Examination

Zifeng Yang, Ph.D.

Rory Roberts, Ph.D.

Philippe Sucosky, Ph.D.

Robert E.W. Fyffe, Ph.D.

Vice President for Research and

Dean of the Graduate School 


\begin{abstract}
Johnson, Mark Bradley. M.S.M.E. Department of Mechanical and Materials Engineering, Wright State University, 2016. Hybrid Particle Image Velocimetry with the Combination of CrossCorrelation and Optical Flow Method.
\end{abstract}

Particle Image Velocimetry (PIV) has been of relevant discussions lately as the equipment used to obtain temporally and spatially resolved flow fields have advanced rapidly. Despite these advancements, the accuracy of evaluating these images have yet to exceed expectations. Current techniques typically utilize one method, either correlation based (frequently) or optical flow (nonfrequently), and both are vulnerable to specific conditions incorporated in the PIV images. Only through the combination of two methods, cross correlation and optical flow, can a technique benefit from the strengths of each method while concealing the flaws each individual method contains. The Hybrid Particle Image Velocimetry method utilizes the fairly unrestricted crosscorrelation method, which can process images that contain particles with relatively large displacements, and the high resolution analysis of the Optical Flow method. Susceptible to large displacements, the Optical flow method is restricted to images with particularly small displacements. Combining the two methods requires the constraints set forth on the Optical flow method to be conserved. Meaning that the Cross-correlation results have to be manipulated into a form applicable for the Optical Flow method. Thus steps such as interpolation, shifting the image, and filtering the image are crucial for transitioning cross-correlation results to optical flow analysis. Validating the accuracy of the Hybrid method was conducted through standard PIV images that encompassed various parameters encountered in PIV. Each set of images were analyzed by the hybrid method and three other commonly-used correlation techniques in order to 
compare the hybrid method's accuracy with current methods. Results confirmed that the Hybrid method is consistently more accurate than the other methods, especially near the boundaries. Additionally, for cases dealing with large particles or small displacement, the Hybrid method attains more accurate results. 


\section{Contents}

1 Introduction

1.1 Other Velocity Techniques

1.1.1 Intrusive Methods

1.1.2 Non-Intrusive Methods

1.2 Particle Image Velocimetry (Cross-correlation)

1.2.1 Technology Advancements

1.3 Optical Flow Method

1.4 Pros and cons for each method

1.5 Importance of hybrid method

2 Methodology

2.1 Cross-correlation

2.2 Optical Flow Method

2.2.1 Horn-Schunck method

2.2.2 Physics-based Optical Flow method

Page

2.3 Hybrid method

2.3.1 Interpolation

2.3.2 Image shift

2.3.3 Filtering the image

2.4 Error analysis

2.4.1 Cross-correlation error

2.4.2 Optical Flow error

3 Numerical evaluation: synthetic particle images through simulation 23

3.1 Flow over a pair of vortex

4 Numerical evaluation: real PIV images

4.1 Standard PIV image (reference)

4.2 Images with Small \& Large Displacement 34

4.3 Low particle concentration $\quad 44$

4.4 Large particle size $\quad 49$

5 Conclusion $\quad 53$

References $\quad 55$ 


\section{List of Figures}

$\begin{array}{lll}\text { Figure Page } & \text { Pas }\end{array}$

1. Cross-correlation Plane 4

2. Particle Displacement within window of Cross-correlation 11

3. Corresponding Weight to Neighboring Points 16

4. Generated Images with Various Characterized Intensity Gradients 25

5. Coordinates of Data Extraction 26

6. Standard PIV Images $\quad 27$

7. Shifted Image 27

8. Vector Fields (Standard) 28

9. Method Comparison at Various X-Intercepts (Standard) 29

10. Method Comparison at Various Y-Intercepts (Standard) 30

11. Average Error (Standard) 31

12. U-Component Error (Standard) 31

13. V-Component Error (Standard) 32

14. Process of Obtaining Final Solution Vectors 33

15. U-Component Error with Respect to Individual methods within the Hybrid 33 method (Standard)

16. V-Component Error with Respect to Individual methods within the Hybrid 34 method (Standard)

17. Small Displacement PIV Images 34

18. Vector Fields (Small Displacement) 35

19. Method Comparison at Various X-Intercepts (Small Displacement) 36

20. Method Comparison at Various Y-Intercepts (Small Displacement) 36

21. Average Error (Small Displacement) 37

22. U-Component Error (Small Displacement) 38

23. V-Component Error (Small Displacement) 39

24. Large Displacement PIV Images 39

25. Vector Plots (Large Displacement) 40

26. Method Comparison at Various X-Intercepts (Large Displacement) 41

27. Method Comparison at Various Y-Intercepts (Large Displacement) 41

28. Average Error (Large Displacement) 42

29. U-Component Error (Large Displacement) 43

30. V-Component Error (Large Displacement) 43

31. Low Particle Concentration PIV Images 44

32. Vector Plots (Low Density) 45

33. Method Comparison at Various X-Intercepts (Low Density) 46

34. Method Comparison at Various Y-Intercepts (Low Density) 46

35. Average Error (Low Density) 47

36. U-Component Error (Low Density) 48 
37. V-Component Error (Low Density) 48

38. Large Particle Size PIV Images 49

39. Vector Plots (Large Particle Size) 50

40. Method Comparison at Various X-Intercepts (Large Particle Size) 50

41. Method Comparison at Various Y-Intercepts (Large Particle Size) 51

42. Average Error (Large Particle Size) 52

43. U-Component Error (Large Particle Size) 52

44. V-Component Error (Large Particle Size) 53 


\section{List of Tables}

Table

1. Average error corresponding to Characterized Intensity Gradient
Page

25 


\section{Acknowledgment}

I would like to extend my gratitude to Dr. Yang for his continuous support throughout this project. Dr. Yang's belief and confidence in my abilities provided me with the motivation and determination needed to complete this work and the work of other classes. I am eternally grateful for all of the opportunities he has provided for me to succeed, not only in the academic and business world but also as a human being.

I would also like to thank the members serving on my defense committee: Dr. Philippe Sucosky and Dr. Rory Roberts. Your comments and advice have improved upon the completeness of this work along with the growth of my critical thinking and presentation skills.

I would like to thank the College of Engineering and Computer Science at Wright State University, more specifically the Mechanical and Materials Science Department, for all of their support. I am appreciative for all of the career and academic opportunities offered throughout my undergraduate and graduate years.

Thank you to my family for the never ending support and encouragement. For always signing forms that enabled me to partake in advanced courses, even when others did not believe in me. Constantly pushing me to accomplish goals that seemed out of reach, at that time. Your love and support does not go without notice.

To the Kesler family, I want to thank you for everything you have done for me these past four years. Your kind and crazy personalities have made Dayton a home away from home for me.

Last but certainly not least, I want to express my appreciation for Lauren Kesler. These past five years have been extremely taxing for me and knowing that you put up with me is an accomplishment in itself. People joke that I helped them survive engineering, but if it wasn't for you I probably would have ended up going crazy. I am forever in your debt. 


\section{Introduction}

The goal of this project is to improve upon the accuracy of Particle Image Velocimetry (PIV) through the use of a hybrid method as the image processing algorithm. The hybrid method entails the use of cross-correlation and the Optical Flow method. Separately the two methods are fairly accurate, however both methods have constraints that limit the procedures regarding how the PIV images are recorded. The hybrid method compromises with these constraints to establish a more forgiving method that also increases the accuracy of the flow field.

\subsection{Other Velocity Techniques}

Before modern technology the method for evaluating a particular flow was through the naked eye. In 1904 Ludwig Prandtl used a water tunnel to observe the effects of a fluid flowing over various objects such as wings (Raffel et al., 2007). Observing the full characteristics of the flow was identified through the use of mica particles. This approach provided insight into the interaction between a fluid and a particular object, but could not obtain quantitative data such as velocity. Ever since then techniques were formed starting with devices that interacted directly with the flow, or intrusive methods, and then evolved into methods that did not interfere with the flow, or non-intrusive methods.

\subsubsection{Intrusive Methods}

Intrusive methods are those that incorporate a measurement device for which interactions with the studied flow are necessary. Two methods are particularly popular: Hot-wire and Pitot tube. Hotwire technology takes advantage of heat transfer, through convection, to establish the velocity at the given point. Current flows through the wire, which in turn generates heat, and the wire can either remain at a constant temperature or maintain a constant current. When a fluid flows over 
the wire, the wire loses heat through convection, thus an increase of voltage is needed to maintain the constant temperature of the wire or the temperature of the wire decreases (Hu, 2011). This change in voltage or temperature is then related to a specific velocity. Other methods incorporate the use of pressure differences between the stagnation pressure and the static pressure. This method is used with Pitot tubes that are arranged within the interrogated flow, where the stagnation pressure is recorded at the location of direct flow contact and the static pressure occurs along the side of the tube. Provided these pressure values, the Bernoulli equation can be applied to calculate the velocity $(\mathrm{Hu}, 2011)$. Although these methods have different approaches, they both have similar outcomes. Calculated velocities are only at one point, therefore the spatial resolution is poor but the temporal resolution is fairly high. Biggest drawback, though, is that these methods disrupt the flow causing certain phenomenon within the flow to change. Thus, alternative methods were developed for more accurate results.

\subsubsection{Non-Intrusive Methods}

Techniques that are able to observe the flow without having to disturb the flow are considered non-intrusive methods. These methods primarily consist of the use of laser technology. The most well-known technique was developed by Yeh and Cummins in 1964 called Laser Doppler Velocimetry (LDV), which was used to study laminar water flow. In LDV, two lasers intersect a

particular region in which particles travel through. When a particle crosses through the intersected region, a wave is reflected and captured by a detector that interprets the frequency of the wave. Velocity is then calculated through comparing the original frequency, of the two lasers, with the frequency of the reflected wave based upon the Doppler effect. Measurements of the velocity can be calculated for consecutive periods of time, however this method deals with velocity components at one specific point (or a very small region). Another method measures the velocity and path line of specific particles of a particular region. This method is called Particle Tracking Velocimetry (PTV), where the results can yield a two- or three-dimensional result, based on the 
experimental setup. Since this method deals with velocities for a specific particle, it lacks the spatial resolution that one would need to determine the flow field of a precise region. Both of these methods could, in theory, calculate a flows velocity field but that would incorporate a large portion of invested time. Not to mention if both of these techniques calculated a flow field then the field would be a time average resolved flow field. This is due to calculating multiple single points at different points in time. Hence, a method that could evaluate a regions' entire velocity field with time resolved flow fields was developed: Particle Image Velocimetry.

\subsection{Particle Image Velocimetry (Cross-correlation)}

Particle Image Velocimetry (PIV) is a technique in which a flow field is filled with marked particles that are captured through successive imaging. PIV includes a thin laser sheet that illuminates the seeding particles within a specific plane while simultaneously recording the image. Additionally, a second pulse is initiated, after the first pulse has disappeared, to capture the succeeding time frame of the image. These images are then used for further analysis to establish an instantaneous velocity field that encompasses the planar section of the observed flow (Quènot et al., 1998). PIV can provide a large spatial resolution with thanks to the advancement of high-resolution cameras, but the time domain is fairly limited due to the laser technology (Raffel et al., 2007). This technique is of growing interest as it is non-intrusive, so for boundary layers near a wall, PIV is highly applicable (Raffel et al., 2007). Other advantages include the capability of measuring and visualizing complicated unsteady flow fields (Hu et al., 1998). These images can be compared through a technique called cross-correlation, which was derived by Adrian in 1988, where the light intensity at two points of time along with a separation vector contributes to the cross-correlation (Adrian \& Keane, 1992). This development leads to the decomposition of the cross-correlation estimator into three components: Convolution of the mean intensities, noise fluctuation, and the correlating displacement (Adrian \& Keane, 1992). These three components are byproducts of the cross-correlation process in which the images are divided 
into equal sized windows for further comparisons. Each window, within the first image, is evaluated inside a search region of the second image. The search region is larger than image one's window in order for the window to move around within the search region therefore providing multiple correlation values. For instance, one can consider a window of $3 \times 3$ pixels that is contained by a $5 \times 5$ search region. The window would be initially placed in the center of the search region where the intensity values are compared. Once a correlation is derived for that set, the window would be moved by one pixel in a step and evaluated again until all possibilities have been compared within the search region. Figure 1 gives a more visual representation of this process.

\begin{tabular}{|c|c|c|c|c|c|c|c|c|c|c|c|c|c|c|}
\hline 12 & 33 & 42 & 5 & 4 & 1 & 32 & 43 & $\begin{array}{ll}52 \\
5\end{array}$ & 4 & 1 & 3 & $\begin{array}{ll}4 & 2\end{array}$ & $\begin{array}{ll}5 & 3\end{array}$ & 42 \\
\hline $\begin{array}{ll}2 & 1\end{array}$ & 23 & 22 & 3 & 2 & 2 & $\begin{array}{ll}2 & 1\end{array}$ & 23 & $\begin{array}{ll}3 & 2\end{array}$ & 2 & 2 & 2 & $\begin{array}{ll}2 & 1\end{array}$ & 33 & 22 \\
\hline $\begin{array}{ll}4 & 3\end{array}$ & 13 & $\begin{array}{ll}1 & 1\end{array}$ & 3 & 2 & 4 & 13 & 13 & $\begin{array}{ll}3 & 1\end{array}$ & 2 & 4 & 1 & $\begin{array}{ll}1 & 3\end{array}$ & 33 & 21 \\
\hline 5 & 2 & 3 & 3 & 1 & 5 & 2 & 3 & 3 & 1 & 5 & 2 & 3 & 3 & 1 \\
\hline 2 & 3 & 1 & 2 & 1 & 2 & 3 & 1 & 2 & 1 & 2 & 3 & 1 & 2 & 1 \\
\hline 1 & 3 & 4 & 5 & 4 & & & & & & 1 & 3 & 4 & 5 & 4 \\
\hline 2 & 2 & 2 & 3 & 2 & & 47 & 51 & 60 & & 2 & 2 & 22 & 33 & 22 \\
\hline 4 & 1 & 1 & 3 & 2 & & 47 & $\ldots$ & 50 & & 4 & 1 & $\begin{array}{ll}1 & 1\end{array}$ & $\begin{array}{ll}3 & 3\end{array}$ & 22 \\
\hline 5 & 2 & 3 & 3 & 1 & & $\ldots$ & $\ldots$ & $\ldots$ & & 5 & 2 & $\begin{array}{ll}3 & 3\end{array}$ & $\begin{array}{ll}3 & 3\end{array}$ & $\begin{array}{ll}1 & 1\end{array}$ \\
\hline 2 & 3 & 1 & 2 & 1 & & & & & & 2 & 3 & 1 & 2 & 1 \\
\hline
\end{tabular}

Figure 1: Cross-correlation Plane

After all of the possibilities are evaluated, the region with the highest correlation coefficient is used as the indicator for how far the particles, for that window, traveled between the succeeding images. This process continues until each window from the initial image has an associated region from the second image. Notice that these displacement vectors are of linear form only, therefore these vectors are strictly limited to first order (Raffel et al., 2007). Cross-correlation is the basis for multiple methods such as the direct correlation method, Hart correlation, and the Fast Fourier Transform (FFT). Direct correlation evaluates the intensity at each pixel within the search region with respect to the window from the first image. However, high correlation values were observed 
with high density regions of particles. Thus, a normalizing scheme was placed in order to associate the equivalent number of particles with the correct displacement in order to avoid regions with large average intensities. Other methods, like the Hart method take on a different approach. The Hart method uses a technique that avoids the dependency associated with the image characteristics as well as the need to correlate small data sets by implementing an algorithm for recursive correlation (Hart, 1999). Principle behind the recursive correlation is to approximate the displacement for a large region that acts as a constraint for correlating the smaller regions within the large region (Hart, 1999). Up to this point the methods mentioned involve the use of correlating intensity levels based on space which requires a large amount of calculations. For a given square window of size $L$, the number of calculations required is $L^{4}$ (Pust, 2000). Therefore a new method was created in order to perform these tasks at a faster rate, hence the FFT. The method converts the intensity in space into a frequency domain. Thus by using the complex conjugate multiplication, the correlation between successive images can be determined (Raffel et al., 2007). One item of concern for these methods, however, is that the displacement is only as accurate as the size of the pixel since the displacement is calculated for an integer value. Analyzing the displacement for a fraction of a pixel is called sub-pixel interpolation. Two methods are of popularity: Center of gravity and Gaussian fit. Center of gravity utilizes the points that correspond to the correlation peak, for which the position of each point is multiplied by the respective intensity and then added together. The summation is then normalized through division of the accumulated intensities. For regions with higher number of identified points, the more accurate the center of gravity method becomes. This particular method does not assume a local maximum point, but an alternative form uses only three identified points with the local maximum peak included (Bastiaans). Other methods, such as the Gaussian fit, utilize the fact that the peak is a function with a central maximum and incorporate the Gaussian function to determine the fractional displacement. 


\subsubsection{Technology Advancements}

Over the years technology has advanced rapidly and with these new advancements there have been significant improvements upon existing techniques. For instance, experimental equipment for PIV testing includes the use of laser and camera technology. Growth in laser technology has allowed for the development of new lasers that feature higher frequencies and consistent power output between pulses. These two advancements alone are crucial to the accuracy of PIV results. For PIV algorithms the intensity is crucial in calculating displacement. If the power output from the first laser pulse to the second differs, then the intensity of the particles also contrast. Therefore a higher level of error is introduced into the calculation of the displacement. Also, with these new laser innovations the intensity difference between images can be minimized and consequently the error. Furthermore, by developing higher frequencies the time in between laser pulses can be minimized, thus causing a smaller time step amongst images. Decreasing the time between images allows for less displacement of the particles. Eliminating large particle movement permits for particle patterns to remain intact. These patterns allow for the PIV algorithms to calculate displacement values. To involve large particle displacements within two images is essentially introducing large portions of error in the results. Improvements in laser technology are inadequate if the camera being used is unable to operate at the same level as the laser. Hence the need for progress in camera technology was equivalently important. In order to capture the laser pulses with higher frequencies, the camera's frame rate had to be improved. Current cameras can reach a quarter of a million frames per second. To put in perspective, the normal motion picture is played back in twenty-four frames per second. Another progressive camera feature is the resolution. Increasing the resolution of a picture allows for more defined images and in effect more accurate results. Recall that the cross-correlation method is a statistical approach. By introducing more pixel values the amount of "sample" correlation values also increases. Therefore when a displacement value is calculated, the size of the pixel is smaller and therefore more concise as opposed to images with lower resolution. Some cameras are able to record images with a 
resolution of 5.5 million pixels, like the LaVision Imager sCMOS. Each of these new developments have contributed greatly to acquiring commendable PIV images, which in turn establishes the foundation to accurate results after being applied to the PIV algorithms.

\subsection{Optical Flow Method}

In 1981, Horn and Schunck first proposed a brightness constraint in order to determine the flow's motion while a variational formulation was used to develop a velocity field, or optical flow, that alters one image into the next through a time sequence (Horn and Schunck, 1981; Liu and Shen, 2008). Use of the brightness constraint assumes that the intensity of the particles are constant from one image to the next. However, this assumption does not hold any physical truth due to the fact that the derivation of the equation does not come from any physical principle (Liu and Shen, 2008). Thus, Liu and Shen (2008), developed experiments for classic flow fields that incorporated shadowgraph and transmittance imaging in density-varying flows, transmittance and scattering of particulate flows, transmittance through transported passive scalar, laser sheet-induced fluorescence, and schlieren for the purpose of deriving projected motion equations (B. Wang et al., 2014). Results yielded a physical meaning that the Optical Flow is proportional to the pathaveraged velocity of fluid or particles in flow visualizations (B. Wang et al., 2014). Improving the accuracy of the optical flow method for PIV use was conducted extensively by Quènot et al. (1998) through the inclusion of Dynamic Programming that divides PIV images into parallel strips that calculate the displacement in the direction of the strips (Quènot et al., 1998). B. Wang et al. (2014) also conducted mathematical analysis of the Optical Flow methods' fundamental equations including the physics-based Optical Flow equation and the Euler Lagrange equation. From the physics-based Optical Flow equation, they developed a theorem that confirms a unique solution of the minimization problem, thus verifying that the minimization preserves discontinuities in a fluid velocity field (B. Wang et al, 2014). Other work includes the study of optical flow with regards to an optimal control problem, where divergence free criteria, for flow 
conservation, is applied that leads to a proof for the optimal conditions for the optical flow (Barbu et al., 2016). Furthermore, the Optical Flow equation shares a common form with the transport equation (Heitz et al., 2010). Most studies involving the improvement of the optical flow method incorporate a parameter for regularizing space as a consequence of the flow's smoothness for a small region, but it has been proven that this approach effects the magnitude of divergence and vorticity field in a negative manner (Corpetti et al., 2005). Liu et al. (2015) compared the optical flow's performance with recognized cross-correlation methods for synthetic images containing Oseen vortex pair within a uniform flow. Results yielded larger error for the correlation methods than the optical flow near the center of the vortex, where the velocity gradient is largest. This indicates that the optical flow can capture sharp changes in the flow, which is of high importance for applications such as mesoscale dynamics with respect to clouds. Mesoscale dynamics are characterized by systems with a range of motion greater than $10 \mathrm{~km}$, therefore satellite imagery is particularly useful when coupled with computer vision programs in order to calculate cloud velocity. However, the techniques were not well adapted to the spatial displacement, thus a method incorporating a correlation method and the optical flow method were used to calculate the cloud flow more accurately (Hèas et al., 2007). Steps were taken further by utilizing the optical flow method with early storm warning systems such as the Short-range Warning Intense Rainstorms in Localized System [SWIRL] for Hong Kong (Liu et al., 2015). Researchers have also been particularly interested with the dynamics of the sun's outer layers as they have been very influential over extreme phenomenon in the solar system. Exploiting the optical flow method for use in understanding the sun's dynamics provided insight to a relationship with the local magnetic field (Hurlburt et al., 2015). Theory involved with this method has been studied extensively as well as validated, which has created multiple opportunities for applications, including PIV, to become more resourceful. 


\subsection{Pros and cons for each method}

Through the use of cross-correlation there are many benefits of using this method. Since the method evaluates multiple pixels for a single window within a given search region, the search region can be expanded to compensate for larger displacements. Thus, for experiments dealing with comparatively large displacements the cross-correlation method can still evaluate the flow, as well as for experiments with relatively large time steps. Since each window is associated with a certain displacement value the overall direction and magnitude of the flow is developed. On the other hand there are drawbacks, such as the fact that the magnitude is not as precise. The results are not as precise since the method is restricted to the size of the window for determining the displacement. Additionally, the size of the window determines the number of resulting vectors calculated. Therefore, for flows with sudden changes the cross-correlation method will not be able to identify these changes with great accuracy. For particles located near the edge of the first image there are significantly large amounts of error due to particles moving out of the image's frame or new particles moving into the frame (Hu et al., 1998).

Contrary to cross-correlation, the Optical Flow method's search regions are modified by the flow and are not fixed window sizes (Quènot et al., 1998). Optical Flow method has advantages as well that includes the use of evaluating the intensity of each pixel. This allows for velocity vectors to be established at each individual pixel, thus allowing for spatially sudden changes to be recognized. Moreover, in calculating each vector, the displacement can be solved for as a fraction of a pixel therefore giving more precision in the results. With these small movements between successive images, there is a reduction in the number of ambiguous vectors (Quènot et al., 1998). The necessity for small displacements between succeeding images is a consequence of the smoothness constraint, therefore the Optical Flow method cannot identify flows with large displacements. 


\subsection{Importance of hybrid method}

With the increasing demand of a more precise but also general method that can uphold precision for multiple situations, a hybrid method is needed. Through the combination of the crosscorrelation method and Optical Flow method these demands can be met. Corpetti et al. have already proposed the use of an altered Optical Flow method in order to compensate for the large displacements, however their approach is based off of a time step (Corpetti et al., 2005). The Optical Flow method has one drawback, which is the necessity for either a small displacement or small time step. Liu and Shen conducted extensive research on the Optical flow method that lead to a comment about "a combination of the correlation-based method and the optical flow method may be more promising" (Liu \& Shen, 2008). By utilizing the generality of the cross-correlation method, particle movement can be found for relatively large movements as well as small displacement, low density, and larger particle size. Results of the cross-correlation provides for the general movement but the method cannot identify sharp turns very well without the use of the Optical Flow method. Another flaw that the cross-correlation method has is how the displacement is calculated. By analyzing windows from one image and comparing within the search region of the other image, particles are clumped into one vector. Thus, the vector represents an average movement of the particles within the window, but in reality the particles could have different movements, as demonstrated in Figure 2. Therefore, two vector components are needed in calculating the final solution: cross-correlation and Optical flow vectors. 


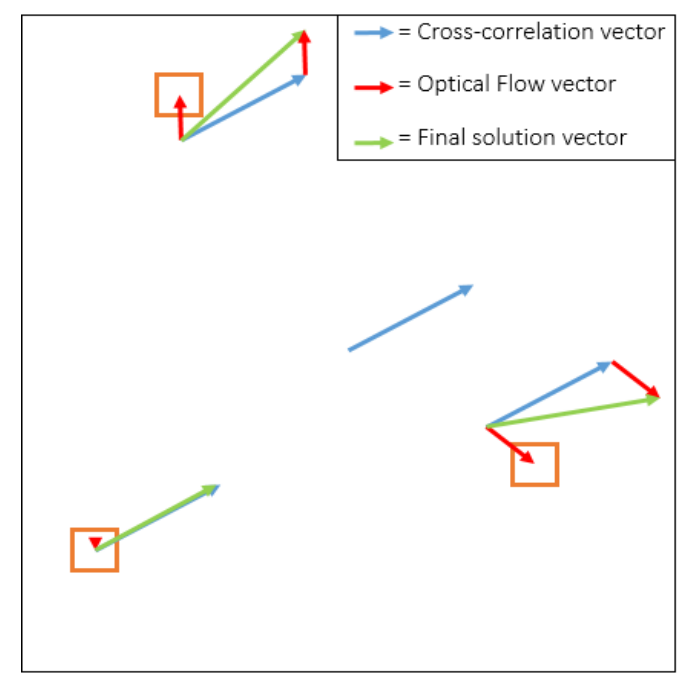

Figure 2: Particle Displacement within window of Cross-correlation

Through use of the Optical Flow method, this error can be countered since the Optical Flow calculates movement based off of each pixel. Thus the precision of the vector field increases and in theory a more precise method for calculating particle velocity while maintaining precision for a large scope of PIV images, including large displacements. This type of method can be effective for projects such as Hèas' et al work contributing to cloud movement from satellite imaging. Hèas et al used a correlation method to analyze the large displacement and further refined the results through the use of a variational optical flow (Hèas et al, 2007). Hence, the need for a hybrid method is of high priority.

\section{Methodology}

\subsection{Cross-correlation}

Cross-correlation is a method derived primarily from a statistical approach. The comparison of a set of pixels in one image with another set of pixels in a second image, is the Cross-correlation method. Recall that as the window, from the first image, moves within the search region of the second image, the intensity values from each pixel are multiplied by the corresponding pixel of the interrogated section of the search region. Each product is summed together to form a value to 
represent how well that particular pattern within the search region represents the displacement. However, some regions include more particles therefore higher intensity values, thus causing a false sense of correlation and more error would be introduced. Avoiding this error can be done through normalizing the intensity of the window and search region through the use of the following equations.

$$
c_{I I}(x, y)=\frac{C_{I I}(x, y)}{\sqrt{\sigma_{I}(x, y)} \sqrt{\sigma_{I}{ }^{\prime}(x, y)}}
$$

Where, $C_{I I}$ represents the convolution of the differences between the intensity of a pixel and the average intensity in the interrogation window in two images, which is described in Equation (2). The variations of intensity distributions within the interrogation window and search region are represented by $\sigma_{I}$ and $\sigma_{I}{ }^{\prime}$, respectively. These equations are displayed in more depth with Equations (3) and (4).

$$
\begin{gathered}
C_{I I}(x, y)=\sum_{i=0}^{M} \sum_{j=0}^{N}\left[I(i, j)-\mu_{I}\right]\left[I^{\prime}(i+x, j+y)-\mu_{I^{\prime}}(x, y)\right] \\
\sigma_{I}(x, y)=\sum_{i=0}^{M} \sum_{j=0}^{N}\left[I(i, j)-\mu_{I}\right]^{2} \\
\sigma_{I}^{\prime}(x, y)=\sum_{i=0}^{M} \sum_{j=0}^{N}\left[I^{\prime}(i, j)-\mu_{I^{\prime}}(x, y)\right]^{2}
\end{gathered}
$$

\subsection{Optical Flow Method}

Deriving the Optical Flow method was credited by Horn and Schunck in 1981. The premise behind the Optical Flow method was initially based off of assumptions and constraints that were not related to any physical meaning. Not until the work of Liu and Shen, did the Optical Flow method contain physics based concepts. Both methods entail the same procedure, in which 
velocity components are calculated for further error analysis and once the error has become minimal the velocity components are selected.

\subsubsection{Horn-Schunck method}

Basis of the Optical Flow method follows the work of Horn and Schunck (1981). Constraints were implemented such as the rate of change in the images' brightness was held constant. Therefore giving the following equation with $I(x, y, t)$ as the intensity and $t$ the time.

$$
\frac{d I}{d t}=0
$$

Through use of the chain rule and letting $u=\frac{d x}{d t}$ and $v=\frac{d y}{d t}$, a linear equation of the two unknowns, $u$ and $v$, was derived.

$$
I_{x} u+I_{y} v+I_{t}=0
$$

Additional partial derivatives were included where $I_{x}, I_{y}$, and $I_{t}$ are taken with respect to $x, y$, and $t$, respectively. Image brightness alone cannot detect the flow's movement, thus a supplementary constraint based upon the smoothness of the flow is introduced. Minimizing the square of the Optical Flow velocity's gradient magnitude is one way of establishing this constraint.

$$
\left(\frac{\partial u}{\partial x}\right)^{2}+\left(\frac{\partial u}{\partial y}\right)^{2} ;\left(\frac{\partial v}{\partial x}\right)^{2}+\left(\frac{\partial v}{\partial y}\right)^{2}
$$

Horn and Schunck's method is based off of the principle of minimizing the amount of error associated with the brightness constraint and the displacement in smoothness within the velocity flow, by optimizing the velocity parameters that create the Optical Flow field. Due to the instability in the brightness constraint introduced by noise and quantization error, a weighting factor, $\alpha$, is provided for the smoothness constraint. Therefore applying more emphasis on the minimization of the smoothness constraint. The total error is represented in the following form. 


$$
\varepsilon^{2}=\iint\left(\alpha^{2} \varepsilon_{c}^{2}+\varepsilon_{b}^{2}\right) d x d y
$$

Where the error associated with the image brightness and the smoothness in the flow are indicated below, respectively.

$$
\begin{gathered}
\varepsilon_{b}=I_{x} u+I_{y} v+I_{t} \\
\varepsilon_{c}^{2}=\left(\frac{\partial u}{\partial x}\right)^{2}+\left(\frac{\partial u}{\partial y}\right)^{2}+\left(\frac{\partial v}{\partial x}\right)^{2}+\left(\frac{\partial v}{\partial y}\right)^{2}
\end{gathered}
$$

The essential factor in minimizing the total error lies within the optimization of the Optical Flow $\operatorname{velocity}(u, v)$. Therefore in order to determine these values the calculus of variation is applied to the total error. Calculus of variation is constructed in the ensuing form.

$$
0=\frac{\partial f}{\partial U}-\frac{\partial}{\partial x}\left(\frac{\partial f}{\partial U_{x}}\right)-\frac{\partial}{\partial y}\left(\frac{\partial f}{\partial U_{y}}\right)
$$

For this particular situation $f$ represents the total error, $U$ denotes the two velocity components $(u, v), x$ is the two directional coordinates $x$ and $y$. Based on these parameters there are two derivations; one for the $u$ component and the second for the $v$ component. First derivation starts with the usage of the velocity component $u$. Breaking the derivation into individual partial derivatives leads to the following solutions.

$$
\begin{gathered}
\frac{\partial f}{\partial u}=2\left(I_{x}^{2} u+I_{x} I_{y} v+I_{x} I_{t}\right) \\
\frac{\partial f}{\partial u_{x}}=2 \alpha^{2}\left(\frac{\partial u}{\partial x}\right) \\
\frac{\partial}{\partial x}\left(\frac{\partial f}{\partial u_{x}}\right)=2 \alpha^{2} \frac{\partial^{2} u}{\partial x^{2}} \\
\frac{\partial f}{\partial u_{y}}=2 \alpha^{2}\left(\frac{\partial u}{\partial y}\right) \\
\frac{\partial}{\partial y}\left(\frac{\partial f}{\partial u_{y}}\right)=2 \alpha^{2}\left(\frac{\partial^{2} u}{\partial y^{2}}\right)
\end{gathered}
$$

Substituting (1212), (14), and (16) into (11) the first derivations' solution is as follows. 


$$
I_{x}^{2} u+I_{x} I_{y} v=\alpha^{2} \nabla^{2} u-I_{x} I_{t}
$$

Deriving the second solution follows the same procedure as the first, however, with the substitution of the $v$ component for the $u$ component. Therefore the solutions of the partial derivatives are of the following.

$$
\begin{gathered}
\frac{\partial f}{\partial v}=2\left(I_{x} I_{y} u+I_{y}^{2} v+I_{y} I_{t}\right) \\
\frac{\partial f}{\partial v_{x}}=2 \alpha^{2}\left(\frac{\partial v}{\partial x}\right) \\
\frac{\partial}{\partial x}\left(\frac{\partial f}{\partial v_{x}}\right)=2 \alpha^{2} \frac{\partial^{2} v}{\partial x^{2}} \\
\frac{\partial f}{\partial v_{y}}=2 \alpha^{2}\left(\frac{\partial v}{\partial y}\right) \\
\frac{\partial}{\partial y}\left(\frac{\partial f}{\partial v_{y}}\right)=2 \alpha^{2}\left(\frac{\partial^{2} v}{\partial y^{2}}\right)
\end{gathered}
$$

The second minimization equation is then produced through the combination of (18), (20), and (22) into (11).

$$
I_{y}^{2} v+I_{x} I_{y} u=\alpha^{2} \nabla^{2} v-I_{y} I_{t}
$$

Obtaining (17) and (23) provides for two equations in order to solve for two unknowns $(u, v)$.

However, both solutions incorporate a Laplacian operator for one of the Optical Flow velocity components. To avoid a complicated derivation, an approximation is created that is of the ensuing form.

$$
\nabla^{2} u \approx K\left(\bar{u}_{i, j, k}-u_{i, j, k}\right) \quad \text { and } \quad \nabla^{2} v \approx K\left(\bar{v}_{i, j, k}-v_{i, j, k}\right)
$$

Local averages $(\bar{u}, \bar{v})$ are determined through a finite numerical method based on neighboring points that are then weighted based off of their location with respect to the evaluated point.

$$
\begin{aligned}
\bar{u}_{i, j, k}=\frac{1}{6}\left(u_{i-1, j, k}+u_{i, j+1, k}+u_{i, j-1, k}\right) \\
+\frac{1}{12}\left(u_{i-1, j-1, k}+u_{i+1, j+1, k}+u_{i+1, j-1, k}\right)
\end{aligned}
$$




$$
\begin{aligned}
\bar{v}_{i, j, k}=\frac{1}{6}\left(v_{i-1, j, k}+v_{i, j+1, k}+v_{i, j-1, k}\right) \\
+\frac{1}{12}\left(v_{i-1, j-1, k}+v_{i+1, j+1, k}+v_{i+1, j-1, k}\right)
\end{aligned}
$$

\begin{tabular}{|c|c|c|}
\hline $1 / 12$ & $1 / 6$ & $1 / 12$ \\
\hline $1 / 6$ & - & $1 / 6$ \\
\hline $1 / 12$ & $1 / 6$ & $1 / 12$ \\
\hline
\end{tabular}

Figure 3: Corresponding Weight to Neighboring Points

Now that a solution is derived for finding the average velocity components, equations (17) and (23) can be solved for the unknowns by finding the determinant of the coefficient matrix. Once the determinant is found, $u$ and $v$ can be solved.

$$
\begin{gathered}
\text { Determinent }=\alpha^{2}\left(\alpha^{2}+I_{x}^{2}+I_{y}^{2}\right) \\
\left(\alpha^{2}+I_{x}^{2}+I_{y}^{2}\right) u=\left(\alpha^{2}+I_{y}^{2}\right) \bar{u}-I_{x} I_{y} \bar{v}-I_{x} I_{t} \\
\left(\alpha^{2}+I_{x}^{2}+I_{y}^{2}\right) v=-I_{x} I_{y} \bar{u}+\left(\alpha^{2}+I_{x}^{2}\right) \bar{v}-I_{y} I_{t}
\end{gathered}
$$

Using (28) and (29) in a numerical method, the Optical Flow velocity can be calculated for each pixel through multiple iterations until the total error is minimized to an optimum value. The iterative solutions of (28) and (29) are listed below.

$$
\begin{aligned}
& u^{n+1}=\bar{u}^{n}-\frac{I_{x}\left[I_{x} \bar{u}^{n}+I_{y} \bar{v}^{n}+I_{t}\right]}{\alpha^{2}+I_{x}^{2}+I_{y}^{2}} \\
& v^{n+1}=\bar{v}^{n}-\frac{I_{y}\left[I_{x} \bar{u}^{n}+I_{y} \bar{v}^{n}+I_{t}\right]}{\alpha^{2}+I_{x}^{2}+I_{y}^{2}}
\end{aligned}
$$

Another characteristic of these equations is that the value calculated at a particular point for one iteration does not directly affect the succeeding iteration for that point. 


\subsubsection{Physics-based Optical Flow method}

Horn and Schunck created the foundation of the Optical Flow method, however, their method was not based on any physical approach. Liu and Shen (2008) developed the Optical Flow method based on a physical meaning and was represented as the following.

$$
\frac{\partial I}{\partial t}+\nabla \cdot(I \boldsymbol{u})=f(x, y, I)
$$

Where $I$ is the intensity and $\boldsymbol{u}$ represents the velocity components $(u, v)$. Using the simplicity of the Horn-Schunck smoothness constraint and the variation formula, an expression is derived.

$$
J(u)=\int_{A}\left[\frac{\partial I}{\partial t}+\nabla \cdot(I \boldsymbol{u})-f\right]^{2} d x d y+\alpha \int_{A}\left(|\nabla u|^{2}+|\nabla v|^{2}\right) d x d y
$$

Where $\alpha$ represents the Lagrange multiplier which is used to emphasize the smoothness constraint over the brightness constraint. Through the use of the Variation of Calculus, four different expressions are derived. First expression is derived from the intensity constraint, where $F=\left[\frac{\partial I}{\partial t}+\nabla \cdot(I \boldsymbol{u})-f\right]^{2}$. Following similar form as used in equation (11) of the Horn-Schunck method,

$$
\frac{\partial F}{\partial u}-\frac{d}{d x}\left(\frac{\partial F}{\partial u^{\prime}}\right)-\frac{d}{d y}\left(\frac{\partial F}{\partial u^{\prime}}\right)=0
$$

Where $u^{\prime}$ represents $\frac{\partial u}{\partial x}$ and $\frac{\partial u}{\partial y}$, respective to the spatial derivative in front. Thus the partial derivatives are expressed in the following.

$$
\begin{gathered}
\frac{\partial F}{\partial u}=2\left(\frac{\partial I}{\partial t}+\nabla \cdot(I u)-f\right)\left(\frac{\partial I}{\partial x}+\frac{\partial I}{\partial y}\right)=2 \nabla \cdot I\left(\frac{\partial I}{\partial t}+\nabla \cdot(I u)-f\right) \\
\frac{\partial F}{\partial u^{\prime}}=\frac{\partial F}{\partial\left(\frac{\partial u}{\partial x}\right)}=2 I\left(\frac{\partial I}{\partial t}+\nabla \cdot(I u)-f\right)
\end{gathered}
$$




$$
\begin{gathered}
\frac{\partial F}{\partial u^{\prime}}=\frac{\partial F}{\partial\left(\frac{\partial u}{\partial y}\right)}=2 I\left(\frac{\partial I}{\partial t}+\nabla \cdot(I u)-f\right) \\
\frac{d}{d x}\left(\frac{\partial F}{\partial u^{\prime}}\right)=2 \frac{\partial I}{\partial x}\left(\frac{\partial I}{\partial t}+\nabla \cdot(I u)-f\right) \\
+2 I\left(\frac{\partial^{2} I}{\partial t \partial x}+\frac{\partial I}{\partial x} \frac{\partial u}{\partial x}+I \frac{\partial^{2} u}{\partial x^{2}}+\frac{\partial u}{\partial x} \frac{\partial I}{\partial x}+u \frac{\partial^{2} I}{\partial x^{2}}+\frac{\partial I}{\partial x} \frac{\partial u}{\partial y}+I \frac{\partial^{2} u}{\partial x \partial y}\right. \\
\left.+\frac{\partial u}{\partial x} \frac{\partial I}{\partial y}+u \frac{\partial^{2} I}{\partial x \partial y}-\frac{\partial f}{\partial x}\right) \\
\frac{d}{d y}\left(\frac{\partial F}{\partial u^{\prime}}\right)=2 \frac{\partial I}{\partial y}\left(\frac{\partial I}{\partial t}+\nabla \cdot(I u)-f\right) \\
+2 I\left(\frac{\partial^{2} I}{\partial t \partial y}+\frac{\partial I}{\partial y} \frac{\partial u}{\partial y}+I \frac{\partial^{2} u}{\partial y^{2}}+\frac{\partial u}{\partial y} \frac{\partial I}{\partial y}+u \frac{\partial^{2} I}{\partial y^{2}}+\frac{\partial I}{\partial y} \frac{\partial u}{\partial x}+I \frac{\partial^{2} u}{\partial x \partial y}\right. \\
\left.+\frac{\partial u}{\partial y} \frac{\partial I}{\partial x}+u \frac{\partial^{2} I}{\partial x \partial y}-\frac{\partial f}{\partial y}\right)
\end{gathered}
$$

Thus, by combining the above equations with (34) the first part is derived.

$$
0=-I \nabla \cdot\left(\frac{\partial I}{\partial t}+\nabla \cdot(I u)-f\right)
$$

Second derivation is found similarly with the slight adjustment of using the other velocity component, $v$. Thus the expression is found with analogous form as (40).

$$
0=-I \nabla \cdot\left(\frac{\partial I}{\partial t}+\nabla \cdot(I v)-f\right)
$$

Remaining portions are derived from the continuity constraint in which $F=\alpha\left(|\nabla u|^{2}+|\nabla v|^{2}\right)$, now, and therefore leads to the ensuing terms.

$$
\begin{gathered}
\frac{\partial F}{\partial u}=\alpha \frac{\partial}{\partial u}\left(\left(\frac{\partial u}{\partial x}\right)^{2}+\left(\frac{\partial v}{\partial y}\right)^{2}\right)=0 \\
\frac{\partial F}{\partial u^{\prime}}=\frac{\partial F}{\partial\left(\frac{\partial u}{\partial x}\right)}=2 \alpha \frac{\partial u}{\partial x} \\
\frac{\partial F}{\partial u^{\prime}}=\frac{\partial F}{\partial\left(\frac{\partial u}{\partial y}\right)}=2 \alpha \frac{\partial u}{\partial y}
\end{gathered}
$$




$$
\begin{gathered}
\frac{d}{d x} \frac{\partial F}{\partial u^{\prime}}=2 \alpha \frac{\partial^{2} u}{\partial x^{2}} \\
\frac{d}{d y} \frac{\partial F}{\partial u^{\prime}}=2 \alpha \frac{\partial^{2} u}{\partial y^{2}}
\end{gathered}
$$

These terms combine and simplify into the following.

$$
0=-\alpha \nabla^{2} u
$$

Second term associated with the new function, $\mathrm{F}$, corresponds to the velocity component, $v$, where the derivation is very similar to before.

$$
0=-\alpha \nabla^{2} v
$$

The Euler-Lagrange equation is now derived, (49), when the four equations are combined: (40), (41), (47), and (48).

$$
I \nabla \cdot\left(\frac{\partial I}{\partial t}+\nabla \cdot(I \boldsymbol{u})-f\right)-\alpha \nabla^{2} \boldsymbol{u}=0
$$

In order to evaluate whether or not the velocity components are accurate, the error due to the intensity $(\Delta I)$ and the error in velocity $(\Delta \boldsymbol{u})$ have to be included. Thus when the errors associated with the intensity and velocity are included into equation (49), we derive an equation for $(\Delta u, \Delta v)$

$$
I \nabla\left[D_{I}+\nabla I \cdot(\Delta \boldsymbol{u})\right]+\alpha \nabla^{2}(\Delta \boldsymbol{u})=\alpha\left(\frac{\Delta I}{I}\right) \nabla^{2} \boldsymbol{u}
$$

Where,

$$
D_{I}=\Delta\left(\frac{\partial I}{\partial t}\right)+\Delta(\nabla I) \cdot \boldsymbol{u}+I \Delta(\nabla \cdot \boldsymbol{u})+\Delta I(\nabla \cdot \boldsymbol{u})
$$

From Equation (50) the error associated with the approximated values for $(u, v)$ can be found. More importantly, Equations (50) and (51) demonstrate how the intensity error is the leading 
cause for calculating inaccurate velocity components. This is non-trivial as the intensity is the only aspect of input for PIV analysis. For example, if the power between two laser pulses are different, then the intensities of the two images will also be different and thus error is already introduced.

Calculating the velocity components are found through multiple iterations. Each iteration incorporates an error between then new $(u, v)$ values and the previous values. If the error is too large then the next set of $(u, v)$ values are calculated through the use of the Euler-Lagrange equation. New approximations are calculated through the discrete scheme described by Liu and Shen, more specifically the Jacobi blockwise iteration method, in the following equations.

$$
\begin{gathered}
\left(I I_{x x}-2 h^{-2} I^{2}-4 \alpha h^{-2}\right) u_{i, j}+I I_{x y} v_{i, j} \\
=I\left(f_{x}-I_{x t}\right)-I\left(2 I_{x}\left(\delta_{x} u\right)_{i, j}+I_{y}\left(\delta_{x} v\right)_{i, j}+I_{x}\left(\delta_{y} v\right)_{i, j}\right) \\
-h^{-2} I^{2}\left(\bar{u}_{i, j}^{x}+\left(\delta_{x y} v\right)_{i, j}\right)-\alpha h^{-2} \bar{u}_{i, j} \\
\left(I I_{y y}-2 h^{-2} I^{2}-4 \alpha h^{-2}\right) v_{i, j}+I I_{x y} u_{i, j} \\
=I\left(f_{y}-I_{y t}\right)-I\left(2 I_{y}\left(\delta_{x} u\right)_{i, j}+I_{x}\left(\delta_{y} u\right)_{i, j}+I_{y}\left(\delta_{y} v\right)_{i, j}\right) \\
-h^{-2} I^{2}\left(\bar{u}_{i, j}^{y}+\left(\delta_{x y} u\right)_{i, j}\right)-\alpha h^{-2} \bar{v}_{i, j}
\end{gathered}
$$

The spatial step is indicated by $h$, which is normally 1 pixel. This process is repeated until the convergence criteria is reached.

\subsection{Hybrid method}

Using the Cross-correlation method will obtain a general sense of the vector field, while the Optical Flow method will be able to calculate a refined vector field capable of identifying sharp velocity changes. However, the need to bridge the two methods together has to be formed. From the results of the Cross-correlation method, the broad vector field has to be interpolated for every pixel. Then, the image is to be shifted through the use of the vectors appointed to each of the pixels in order to provide for a small displacement from the shifted image to the second image. 
From there, the image is filtered for smoother intensity gradient. Through these steps the Crosscorrelation method and the Optical Flow method are able to be combined.

\subsubsection{Interpolation}

Results obtained from the Cross-correlation method are calculated vectors from a group of pixels. Optical Flow method calculates a vector for each pixel, therefore the pixels without vectors have to have a vector appointed to them. Accomplishing this is done through a linear interpolation between the points that already have a vector associated with them.

\subsubsection{Image shift}

Once a vector is associated with each pixel, the image is to be shifted. Reasoning behind this process is that the displacement of the particles are assumed to be too large for the Optical Flow method. Since one of the constraints appended to the Optical Flow method is the smoothness constraint, the particles have to come within one pixel of displacement for best results. Therefore by moving a pixel, of the second image, a distance supplementary to the vector of the pixel, the pixel can be compared to the first image. Every pixel undergoes this process and creates a shift in the second image, thus the smoothness constraint remains intact as the shifted image is compared with the first image.

\subsubsection{Filtering the image}

Shifting the second image creates a new image that is more comparable with the first image, for the purposes of the Optical Flow method. Now the smoothness constraint has been fulfilled, but the constraint dealing with the intensity gradient is yet to be satisfied. To uphold this constraint, a Gaussian filter is applied to both the shifted image and the first image. Filtering the images provides for a smoother intensity gradient from particle regions to regions without particles. As well as allows for a cluster of particles to be more distinguished for the Optical Flow method. All constraints related with the Optical Flow method have been compensated and now the images are in an optimal form for the Optical Flow method. 


\subsection{Error analysis}

Each method has their strengths and weaknesses when evaluating PIV images, therefore to fully understand the risk behind the Hybrid method it is wise to understand the error associated with each of the methods. Defining the parameters of PIV images are important as they can significantly impact the results as well. Main parameters being discussed will be the particle's displacement, density within the interrogation area, and size of the particles in the image.

\subsubsection{Cross-correlation error}

Recall, that the procedure for the Cross-correlation method is to analyze a group of pixels in order to identify similar patterns of intensities. By taking groups of pixels and comparing them to search regions within the second image, there are benefits and disadvantages of this method based on the type of PIV images taken. One situation that could occur is that the intensity from one image to the next is different. If the second image has an average intensity that is greater than the first then the correct displacement could possibly have a lower correlation value than another due to the intensity being higher throughout. Studies were conducted by Hu et al. (1998) that incorporated the size of the interrogation window, size of the search region, particle size, displacement, and the number of particles. From their research, conclusions were drawn which stated that when the number of particles are low then the accuracy of Cross-correlation is poor, but when the number of particles increase the accuracy improves. Similarly, when the size, or diameter, of the particles increase, the accuracy of the Cross-correlation method also improves. For situations where the particles don't move very far from one image to the next, the accuracy suffers even though the correlation values are high. The reason for relatively large error (18.94\%), is due to the restricted resolution of the image (Hu et al., 1998). On the other hand, if the displacement of the particles become too large, then the error increases significantly (55.41\%). When the particles have too large of displacement it allows for patterns observed in the first image to disperse and change in the second image. Another aspect of the error comes from 
the particles traveling outside of the laser emitted plane or new particles traveling into the plane. From the out-of-plane movements, the Cross-correlation method looks for particles that are no longer visible along with having to deal with new patterns from the particles moving into the plane.

\subsubsection{Optical Flow error}

From the error analysis of the physics based Optical flow, equations (50) and (51) give an insight as to the error involved for calculating the Optical flow. The components that influence the error the greatest are of the following: $\Delta\left(\frac{\partial I}{\partial t}\right), \Delta(\nabla I), \Delta(\nabla \cdot \boldsymbol{u})$, and $\Delta I$ (Liu \& Shen, 2008). These components are primarily from equation (51). Since the brightness constraint is of high

importance, it is easy to identify the change in the intensity gradient with respect to time, $\Delta\left(\frac{\partial I}{\partial t}\right)$, as a large component of the error. If the intensity gradient between the first and second image are different, then the comparison of the intensity gradients will be impacted tremendously. Another component of the intensity gradient occurs with respect to the $\mathrm{x}$ - and y-directions. By introducing intensity error along these coordinates, inaccurate results will occur due to the intensity gradient. The change in the velocity gradient is a large part of the error as well, as it represents the ability to comply with the smoothness constraint. Regarding PIV parameters, the error for the Optical flow increases with the increase of displacement due to the solution of the time derivative becoming no longer accurate (Liu \& Shen, 2008). When the displacement is very small, though, Liu and Shen found that the Optical flow method strives when patterns are nearly continuous.

\section{Numerical evaluation: synthetic particle images through simulation}

Optical flow method was developed under the constraint of smoothness, as stated earlier, and in order to establish smoothness within the hybrid method a Gaussian filter is applied. Parameters for the Gaussian filter, however, were never correlated to the accuracy of the Optical flow. Thus 
it was imperative to conduct a study between the Gaussian filter parameters and the error associated with these said parameters for use in the Optical flow method.

\subsection{Flow over a pair of vortex}

This study incorporated a single PIV image which was later used to generate a successive PIV image. Yang et al. (2016) developed a program to create a second image that resembled a uniform flow through which a pair of vortex were generated by manipulating the PIV image. Then, both images went through a Gaussian filter of equal parameter values. To identify the difference between various parameters for the Gaussian filter, a characterized intensity gradient, $\gamma$, was derived.

$$
\gamma=\sum_{i=1}^{n_{x}} \sum_{j=1}^{n_{y}}\left(I_{x}^{2}+I_{y}^{2}\right)^{\frac{1}{2}}
$$

The intensity gradients in the $\mathrm{x}$ - and $\mathrm{y}$ - directions are presented as $I_{x}$ and $I_{y}$, respectively, and the number of pixels in the $\mathrm{x}$ - and $\mathrm{y}$ - direction are represented by $n_{x}$ and $n_{y}$, respectively. Central differencing scheme was used in the calculation of the intensity gradients. For the reason that the second image was generated, the exact solution was known and therefore used in analyzing the error associated with optical flow corresponding to the characterized intensity gradient. Figure 4 displays the generated images that correspond to the characterized intensity gradients:

(a) $\gamma=2.9$; (b) $\gamma=6.1$; (c) $\gamma=13.4$; (d) $\gamma=17.2$.

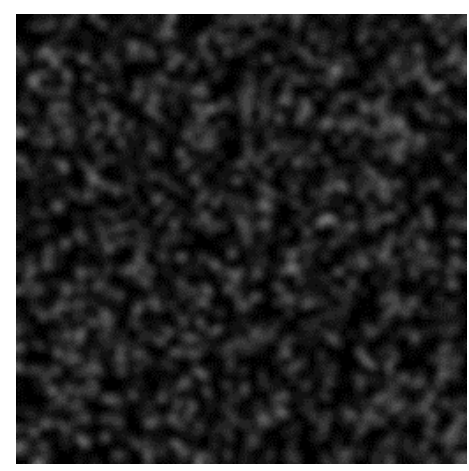

(a)

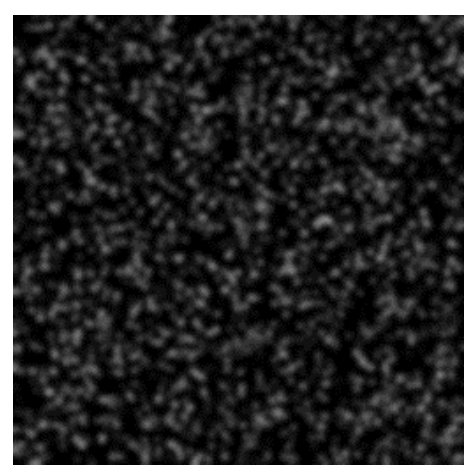

(b) 


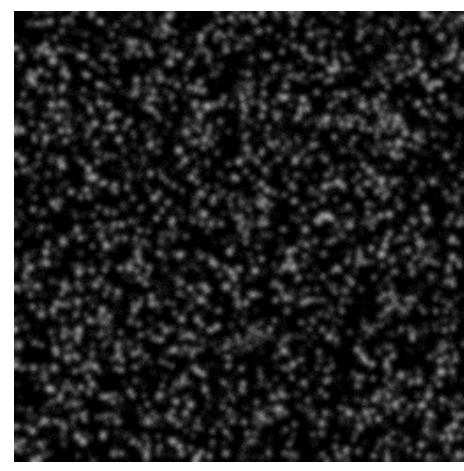

(c)

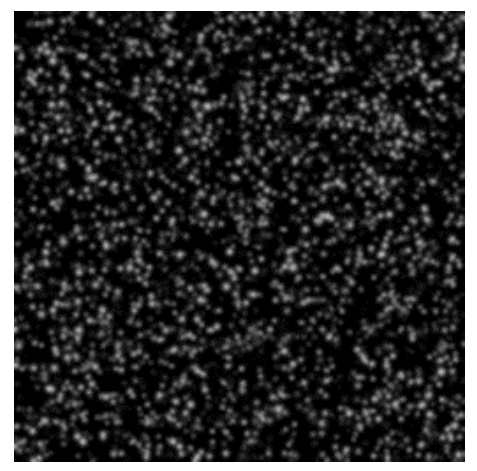

(d)

Figure 4: Generated Images with Various Characterized Intensity Gradients

In Table 1 the Gaussian parameters (mask size and standard deviation) are shown with the corresponding characterized intensity gradient as well as the error associated with each velocity component.

Table 1: Average error corresponding to Characterized Intensity Gradient

\begin{tabular}{|c|c|c|c|c|c|c|}
\hline $\begin{array}{c}\text { Intensity } \\
\text { Gradient, } \gamma\end{array}$ & $s_{1}$ & $\sigma_{1}$ & $s_{2}$ & $\sigma_{2}$ & Error $_{u}$ & Error $_{v}$ \\
\hline 2.9 & 12 & 4.8 & 5 & 2.0 & 2.90 & 2.40 \\
\hline 6.1 & 8 & 3.2 & 3 & 1.2 & 0.87 & 0.73 \\
\hline 9.3 & 5 & 2.0 & 3 & 1.2 & 0.48 & 0.40 \\
\hline 13.4 & 3 & 1.2 & 3 & 1.2 & 0.43 & 0.33 \\
\hline 17.2 & 3 & 1.2 & N/A & N/A & 0.51 & 0.37 \\
\hline
\end{tabular}

Results of this analysis gives insight into two conclusions. One, for PIV images that incorporate very large intensity gradients the amount of error tends to increase. Two, when a Gaussian filter is applied to generate an intensity gradient of 13.4 the error is minimized. Therefore when analyzing particle images with optical flow, cases that incorporate an intensity gradient of 13.4 , with the help of the Gaussian filter, are highly desirable.

\section{Numerical evaluation: real PIV images}

Justifying the validity of the Hybrid method was done through the comparison of exact measurements that encompassed different PIV imaging scenarios: Standard particle image, image with small displacement, image with large displacement, image with low particle density, and image with large sized particles. Other methods such as direct correlation, Fast Fourier 
Transform, and the Hart correlation, from the commercial program Insight 4G, were used to analyze the PIV images for comparison with the Hybrid method's results. Results from each method were extracted along several straight lines crossing the image as shown in Figure 5.

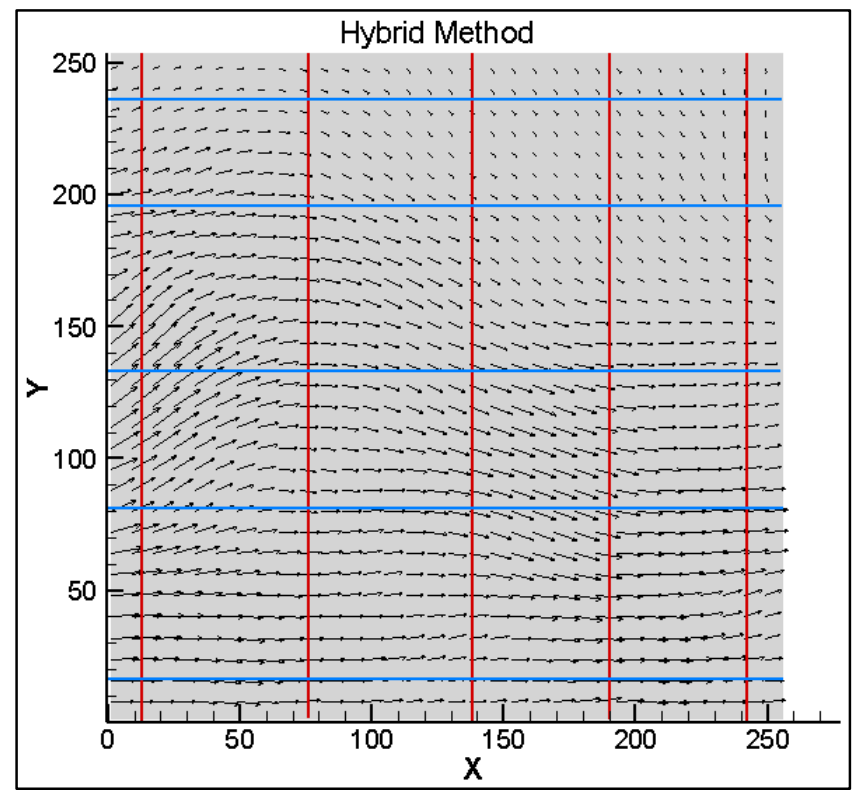

Figure 5: Coordinates of Data Extraction

Data was extracted from the x-intercepts $(16,76,138,190$, and 239) and the y-intercepts $(16,81$, 133, 196, and 236). The average error associated with the velocity in the x-direction (Ucomponent) and in the y-direction (V-component) for each intercept was plotted to show the accuracy of both component. Images were provided by the work of K. Okamoto et al. (2000) with the collaboration of the Visualization Society of Japan that also contributed the exact solution of the flow field. Tables were posted on their website (http://www.piv.jp) that included the average magnitude of the flow along with the number of particles and other parameters.

\subsection{Standard PIV image (reference)}

The standard PIV image is the ideal type of image that one would capture from an experiment.

The number of particles are fairly high to identify particle patterns but not too high where patterns are not distinguishable and the displacement of the particles are not very large. Below are the two images used for this analysis. 

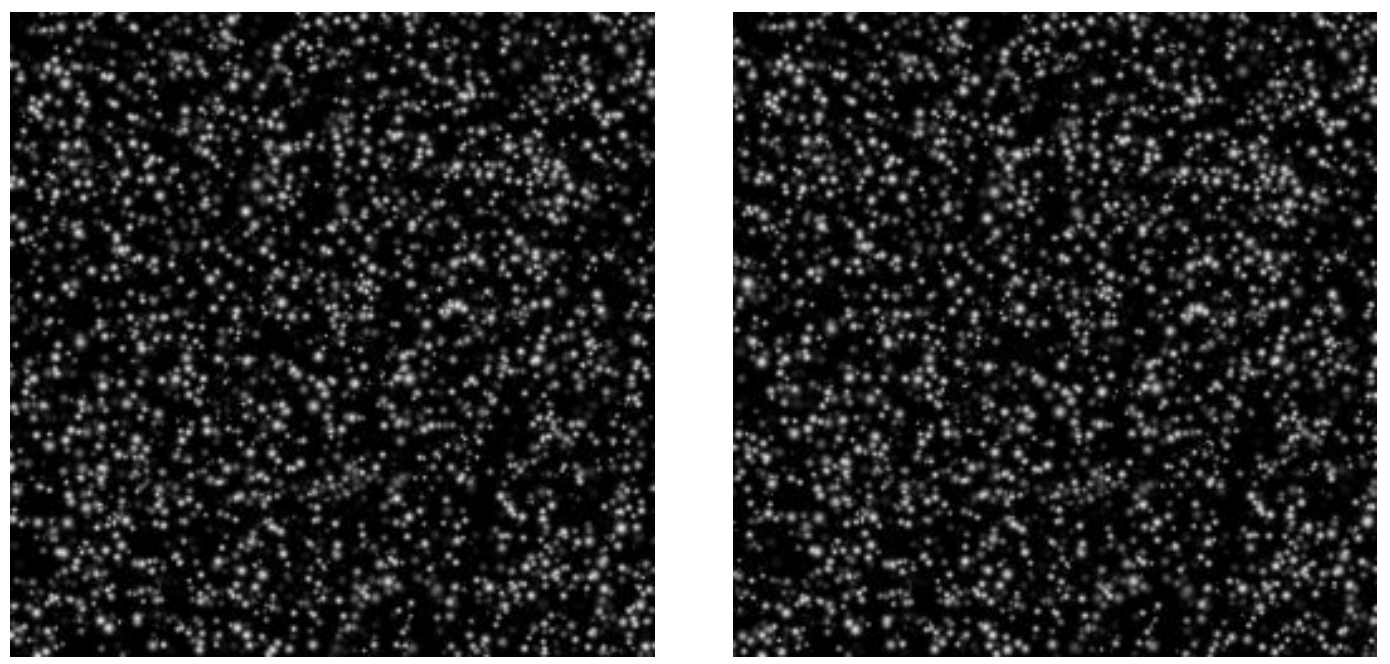

Figure 6: Standard PIV Images

Through the hybrid method an additional image, the shifted image, is created in order for the image comparison to uphold the smoothness constraint associated with the Optical flow.

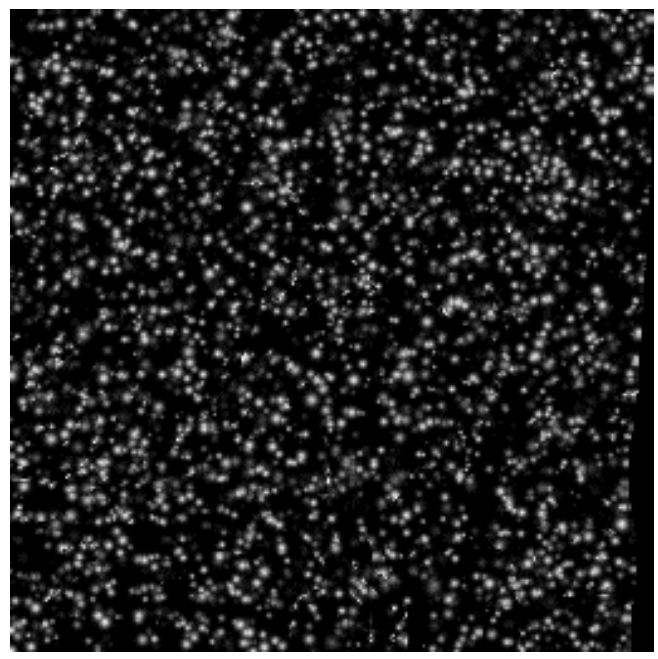

Figure 7: Shifted Image

Figure 7 displays the result of the shifted image, however the shifted image was created by

shifting the second image backwards in time. Reasoning behind shifting the second image is due to the nature of the current flow being studied in this paper, which primarily travels from left to right. In fact, either way for the shifting will not affect the final calculation results except for the boundary. By shifting the second image, the particles on the far left side of the image are all present since the particles from the second image are shifted to the left. Whereas the right end of 
the image contains a region of empty space due to the shift of the image, therefore the accuracy of the optical flow method will decrease near the right side of the image. Increasing the error near the boundary on the right side was reasonable due to the fact that most of the error would occur from particles traveling out of the images frame.

Each of the methods' vector field plots were compared but only the solution, hybrid, and direct correlation are in the following figures. Note that the hybrid method has a vector corresponding to each pixel, but in order for the vector field to be observed 8 vectors in each direction are skipped.
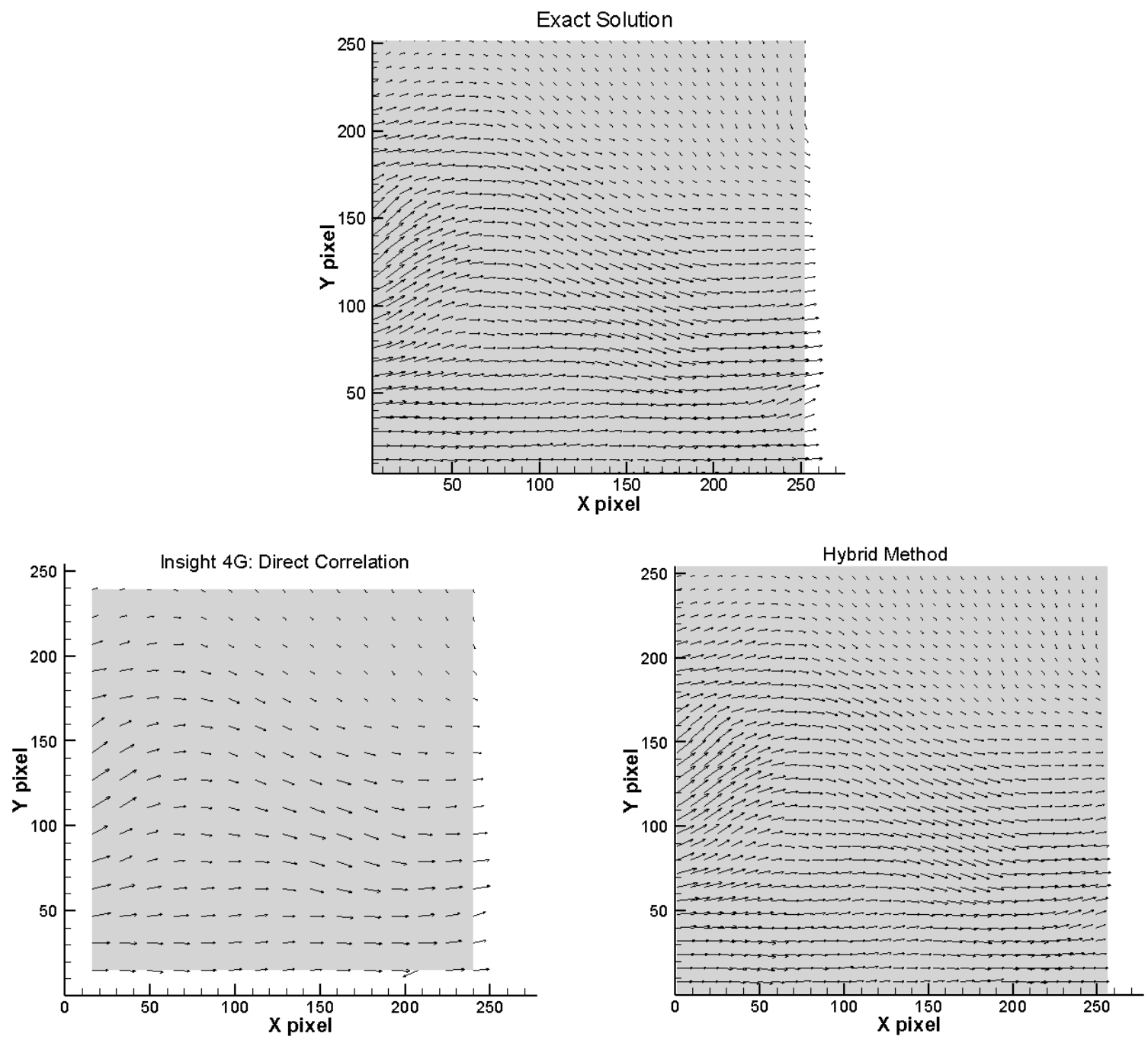

Figure 8: Vector Fields (Standard) 
Through observation of the vector fields, all of the methods tend to have similar flows, but the Direct Correlation (interrogation window of $32 \times 32$ pixel, $50 \%$ overlap) had the best representation of the flow, in comparison to the other correlation methods. There are some vectors that have obvious differences, such as the vector in the bottom right corner of the Direct Correlation but other vectors have small variations. To identify more subtle changes, plots were created based off of the extracted data that lied within the intercepts identified earlier.

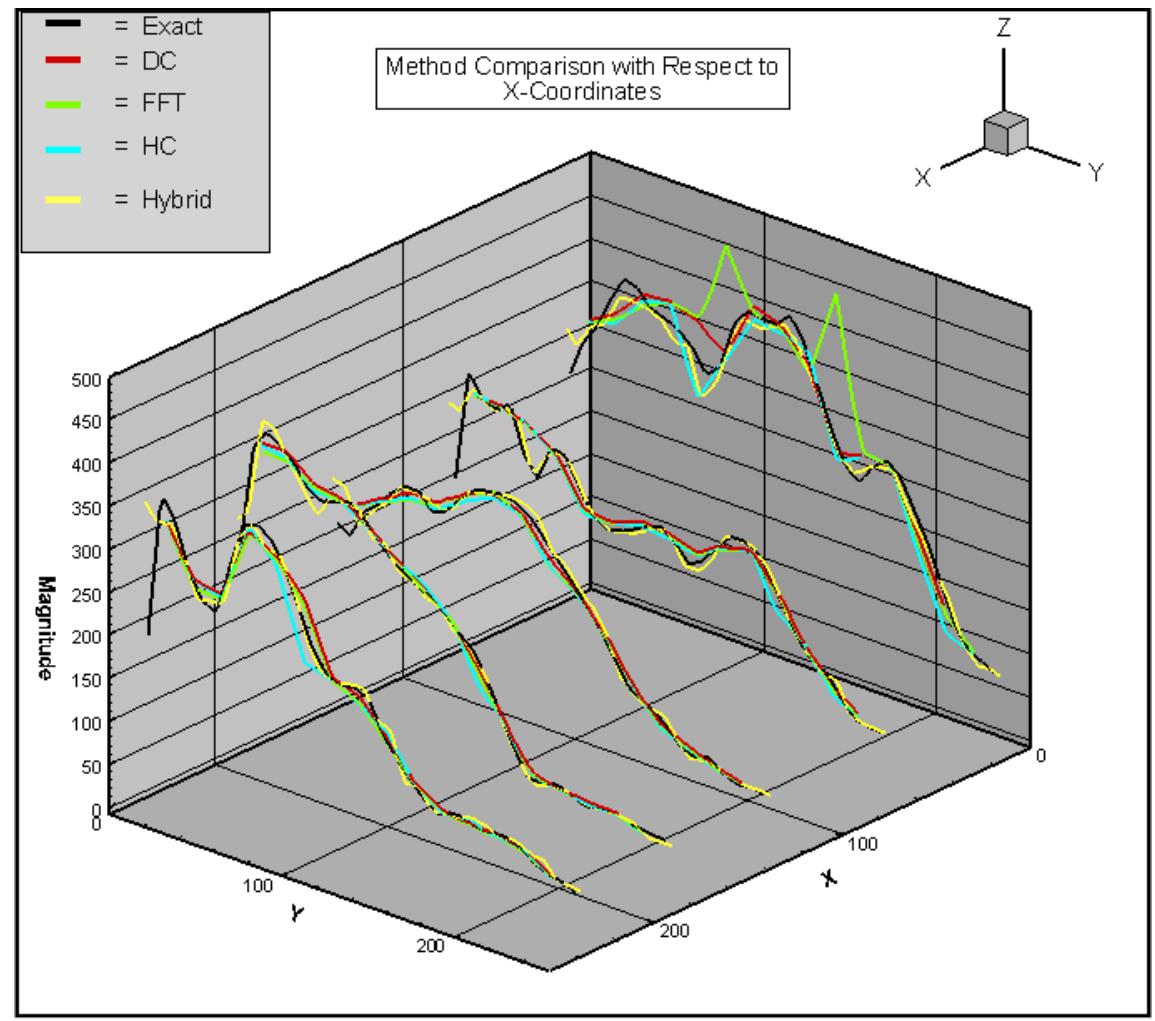

Figure 9: Method Comparison at Various X-Intercepts (Standard) 


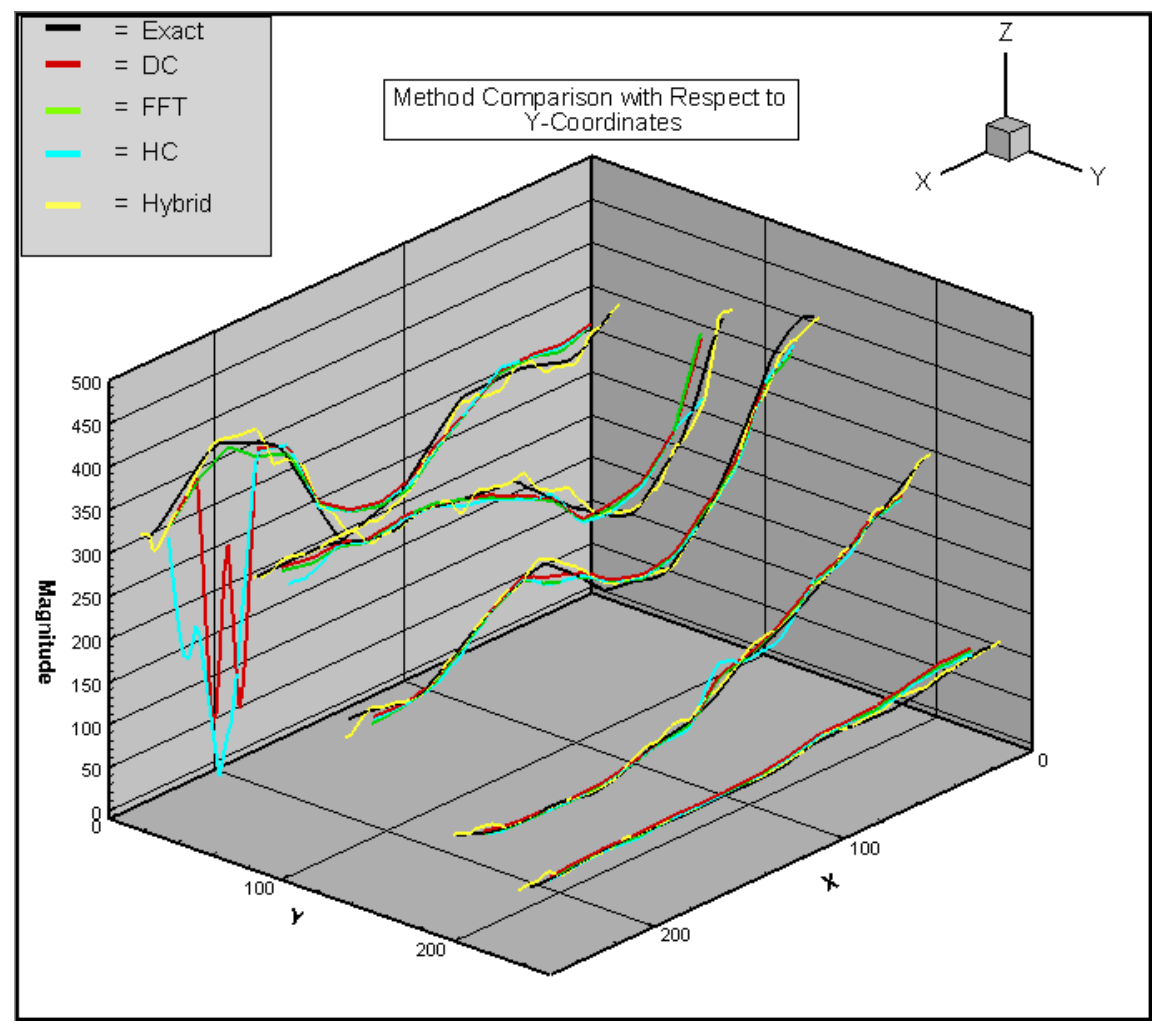

Figure 10: Method Comparison at Various Y-Intercepts (Standard)

The plots shown in Figure 9 and Figure 10 allow for specific regions to be observed in more depth to distinguish the reasoning behind the errors. However these plots alone cannot be used to declare which method performs more accurate than the rest. Thus, average error plots were generated as seen in Figure 11.
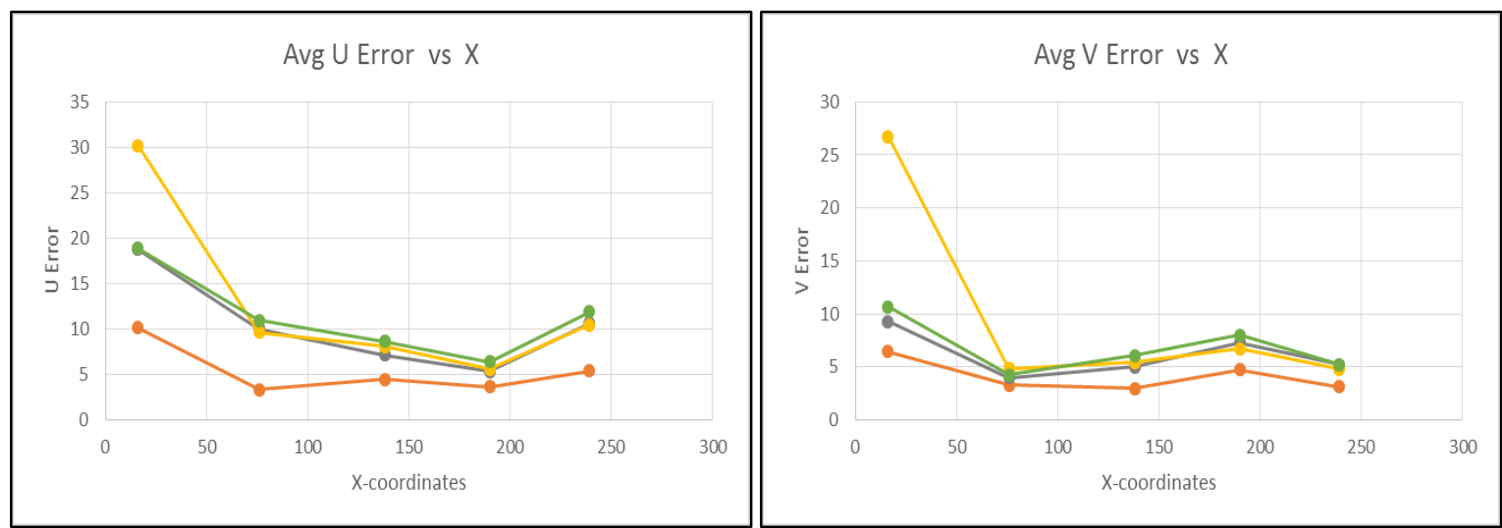


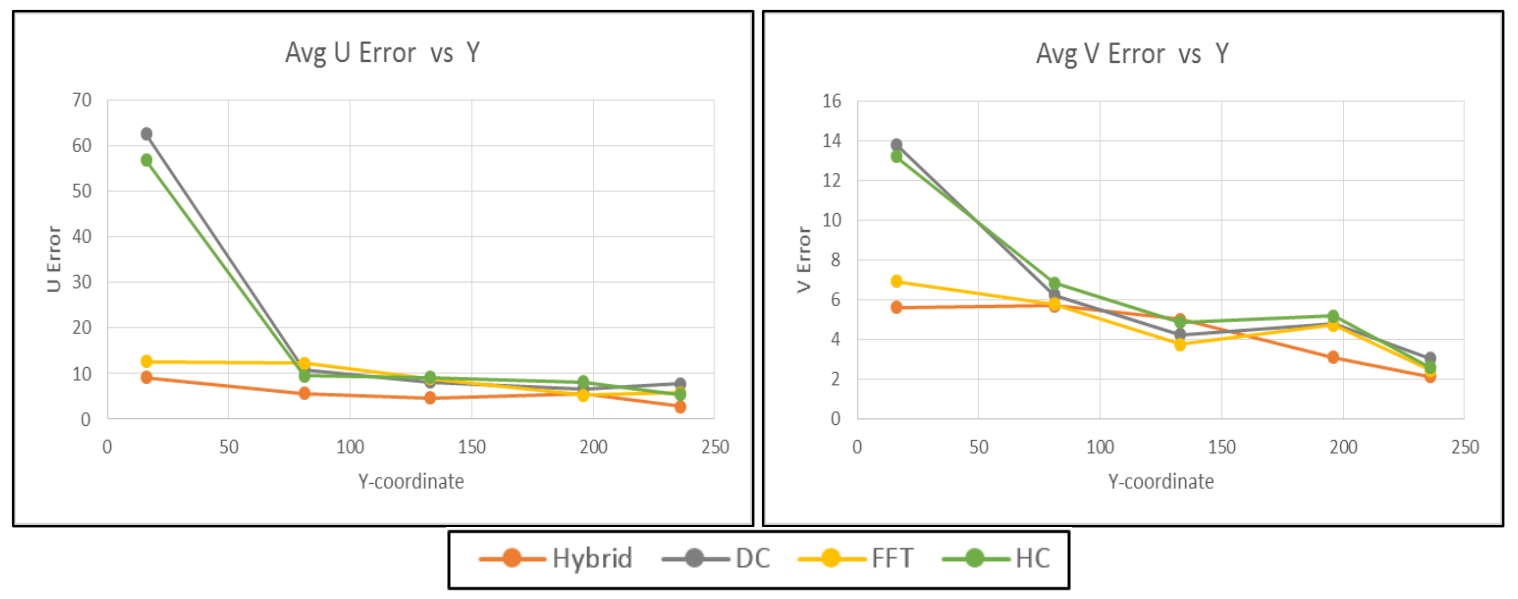

Figure 11: Average Error (Standard)

Based off of the average error associated with the U-component, the hybrid method's error is consistent throughout the $\mathrm{x}$ - and y-coordinates as well as consistently lower, if not equal, than the three other methods. As for the V-component, the hybrid method still performs well through the $\mathrm{x}$-coordinates, but through the $\mathrm{y}$-coordinates the amount of error is fairly similar to the rest of the methods. Generally the hybrid method calculates vectors near the boundary of the image plane with more accuracy than the other methods for the standard image. From these results the Direct Correlation method performed better than the other correlation methods, therefore a 3D error plot was created to compare with the hybrid method.
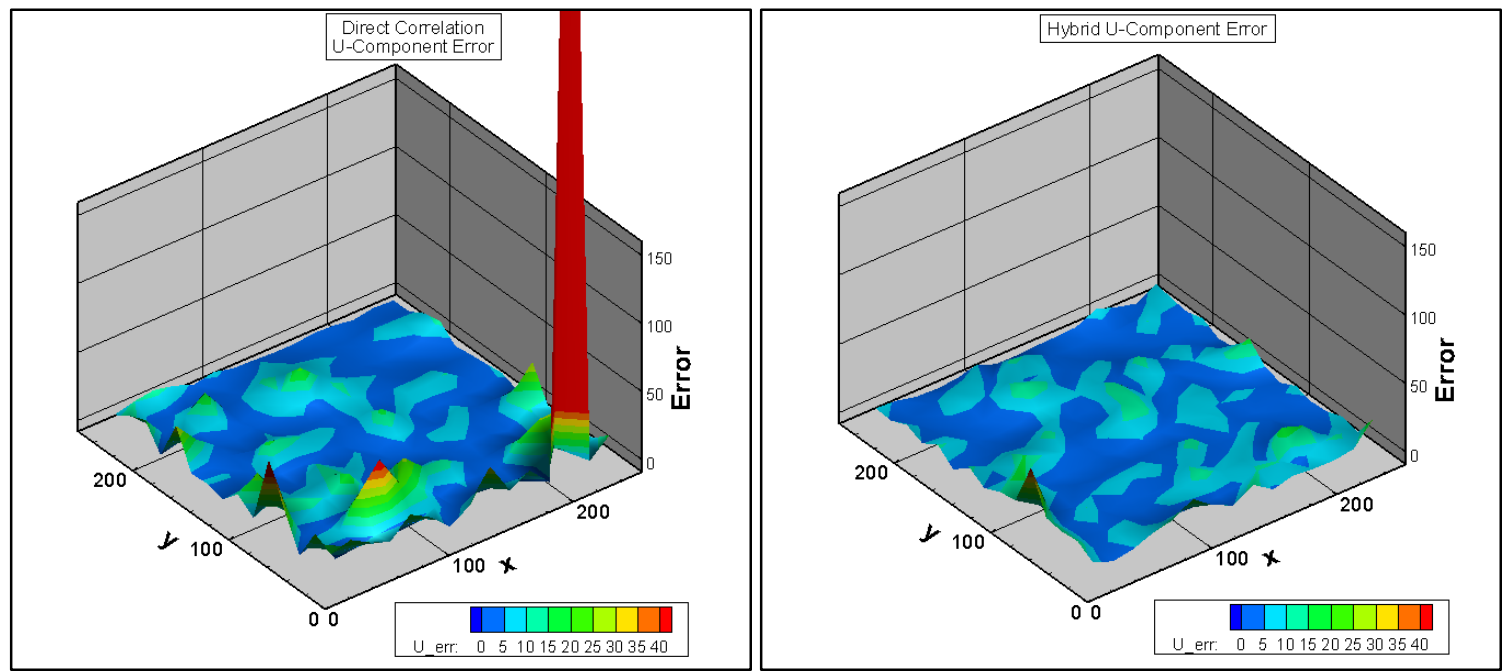

Figure 12: U-Component Error (Standard) 
It is clear from the U-component error plots, above, that the hybrid method incorporates less error. Direct correlation displays poor results near the boundary of $y=0$ as well as $x=0$. Other regions of error are comparable with the hybrid method. The following plots represent the error associated with the V-component for the Direct and hybrid method, respectively.
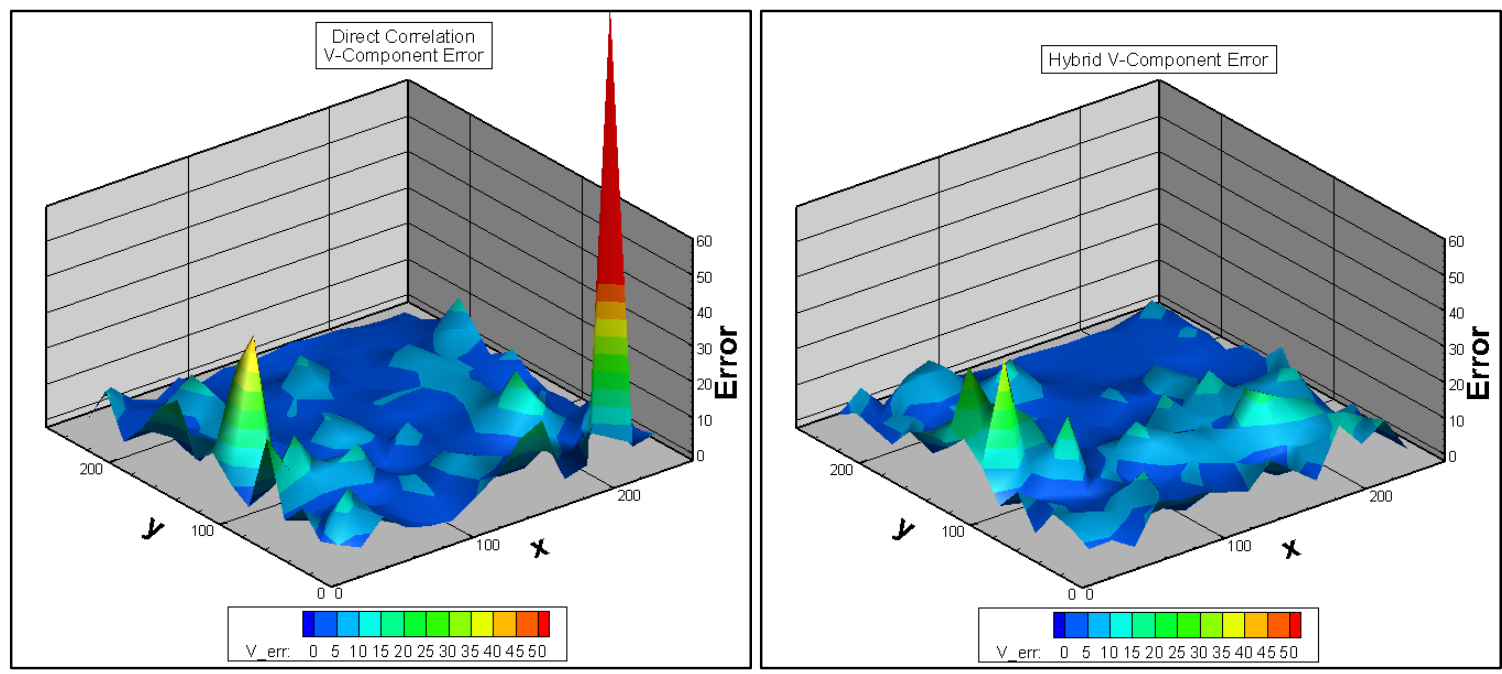

Figure 13: V-Component Error (Standard)

For the V-component, the error related to the Direct Correlation method is fairly low with two sources of major error. Comparing to the Direct Correlation, the hybrid method performs better in most of the regions but does have tendencies for calculating values with error that resemble the error associated with the direct correlation. To understand the hybrid method further, the vector plots from the cross correlation, optical flow, and the combination are shown below.
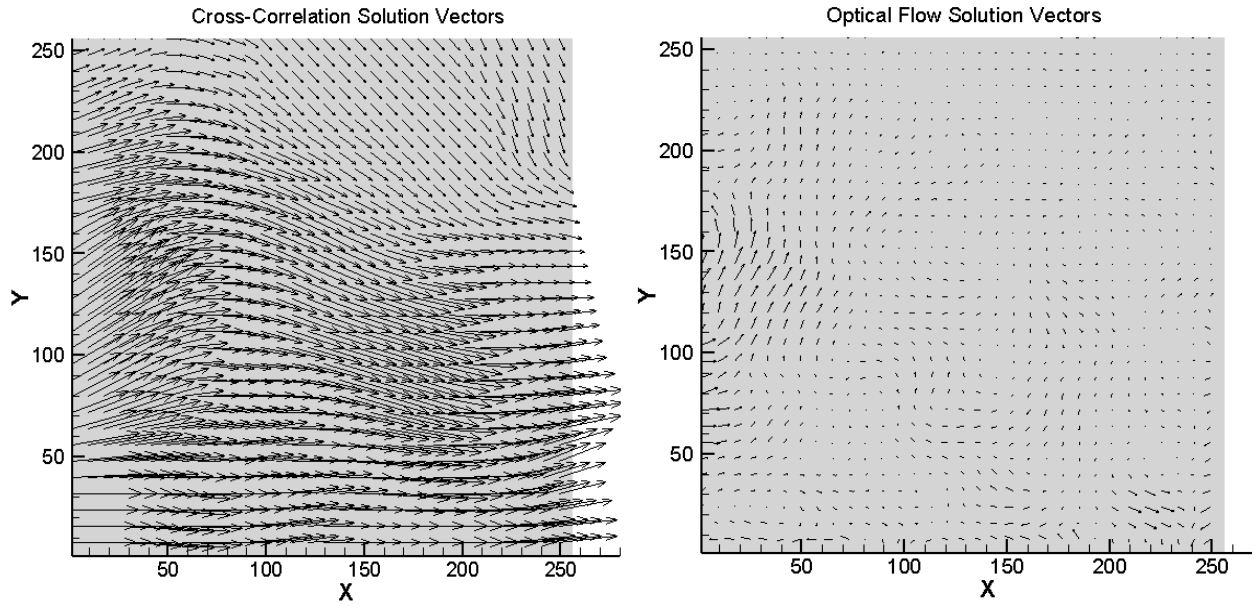


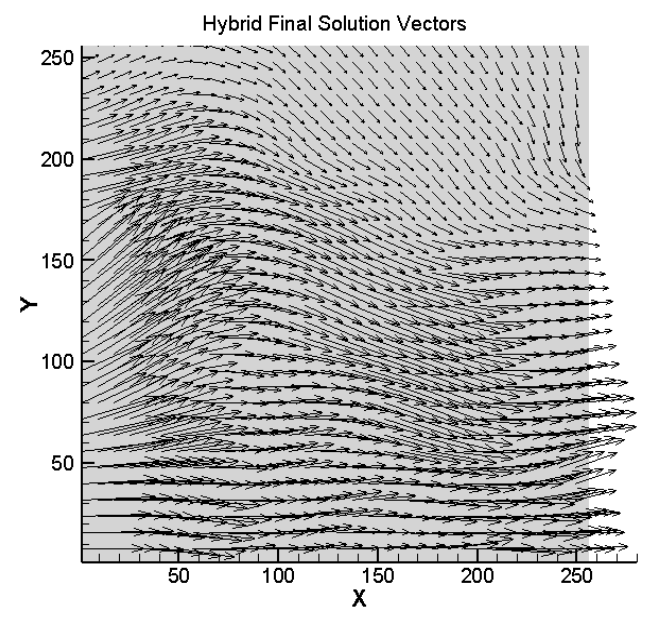

Figure 14: Process of Obtaining Final Solution Vectors

Notice how the macro component of the velocity vector is calculated through the correlation method while the finer vector is found through the optical flow method. By combining the two vectors together a more precise vector field can be obtained. Theoretically the optical flow should reduce the error, therefore the following error plots were created to verify that this was true.
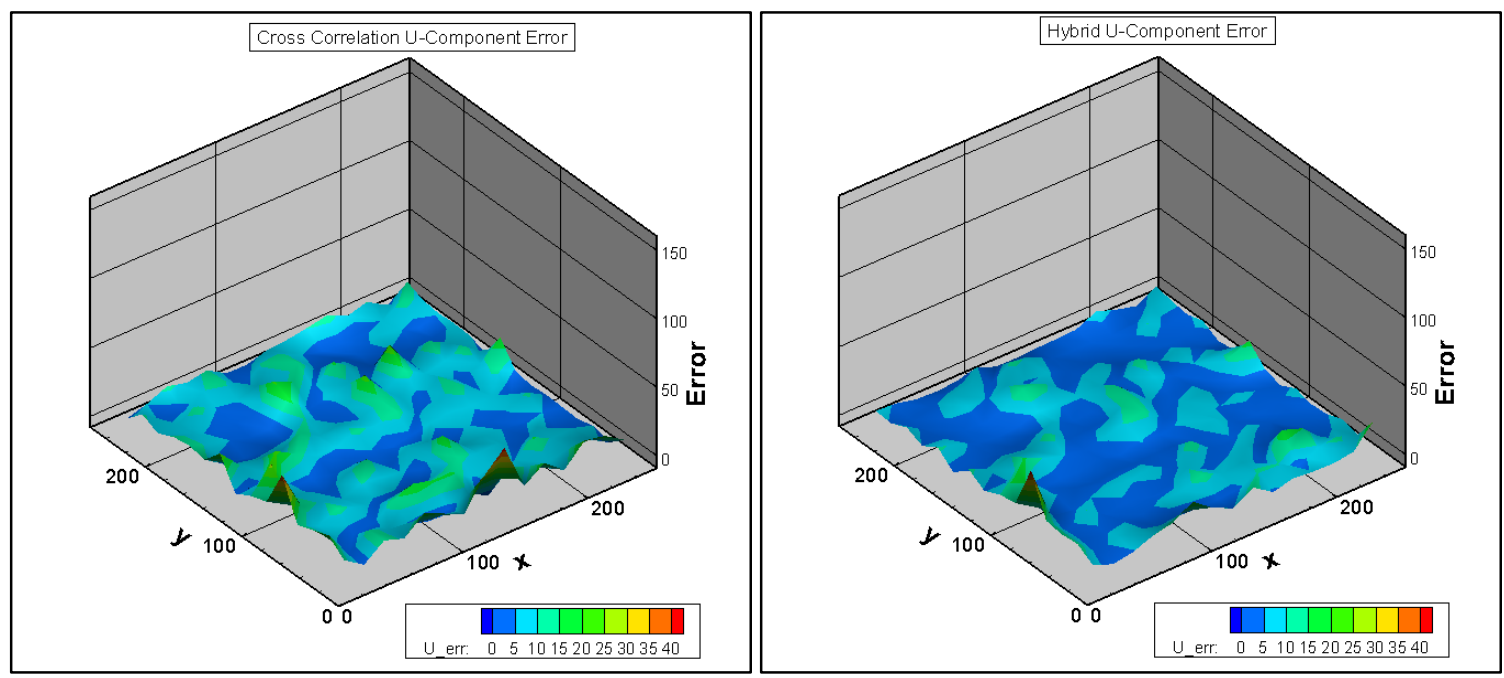

Figure 15: U-Component Error with Respect to Individual methods within the Hybrid method (Standard)

Observing the regions of larger error, it is clear that the cross-correlation method introduces the large portions of error where the addition of the Optical flow method reduces some of the error. The error associated with the calculated V-components also experienced similar results, which can be observed in the following figures. 

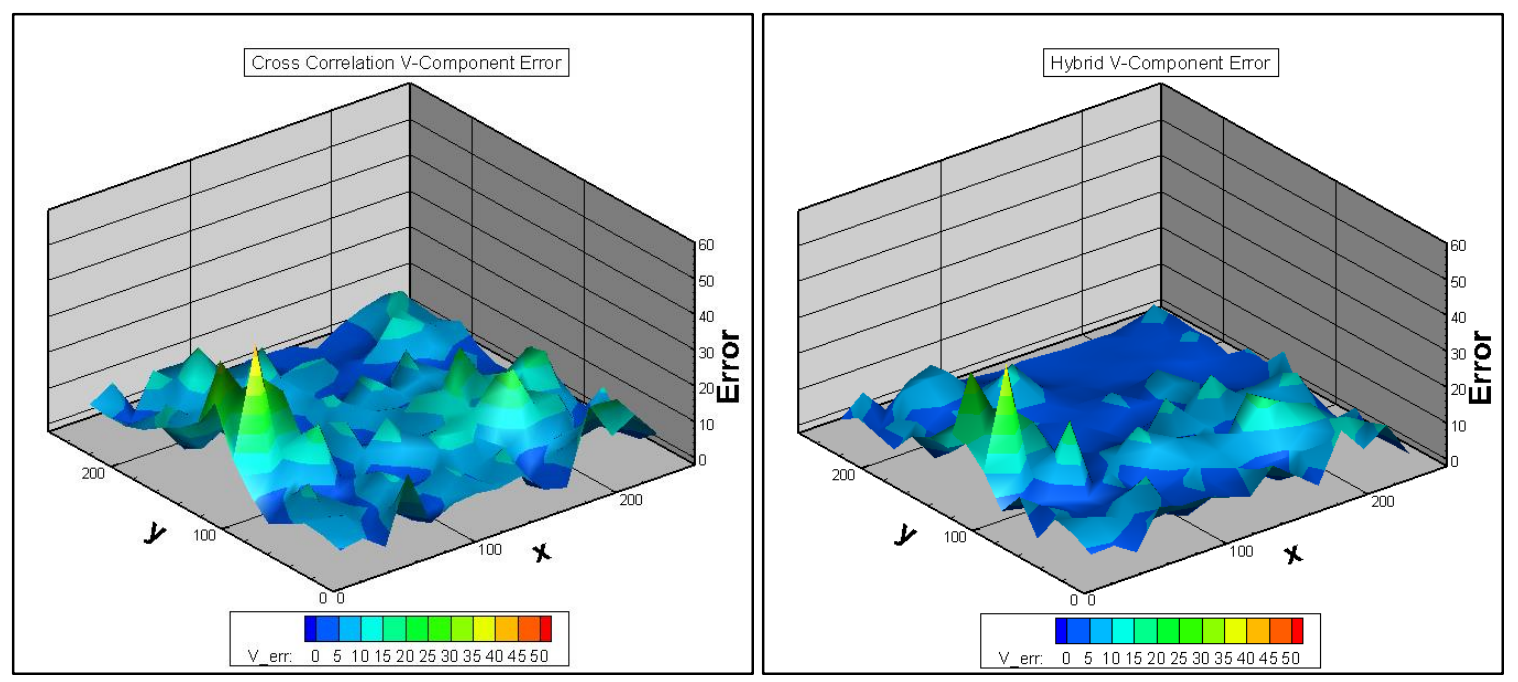

Figure 16: V-Component Error with Respect to Individual methods within the Hybrid method (Standard)

\subsection{Images with Small \& Large Displacement}

For times when the flow is relatively slow or when the time interval in between images is very short, the amount of displacement that the particles undergo is very small from one image to the other. The images used for small displacement were configured by decreasing the magnitude of the flow with respect to the standard images' magnitude. The images of the small displacement are shown below.
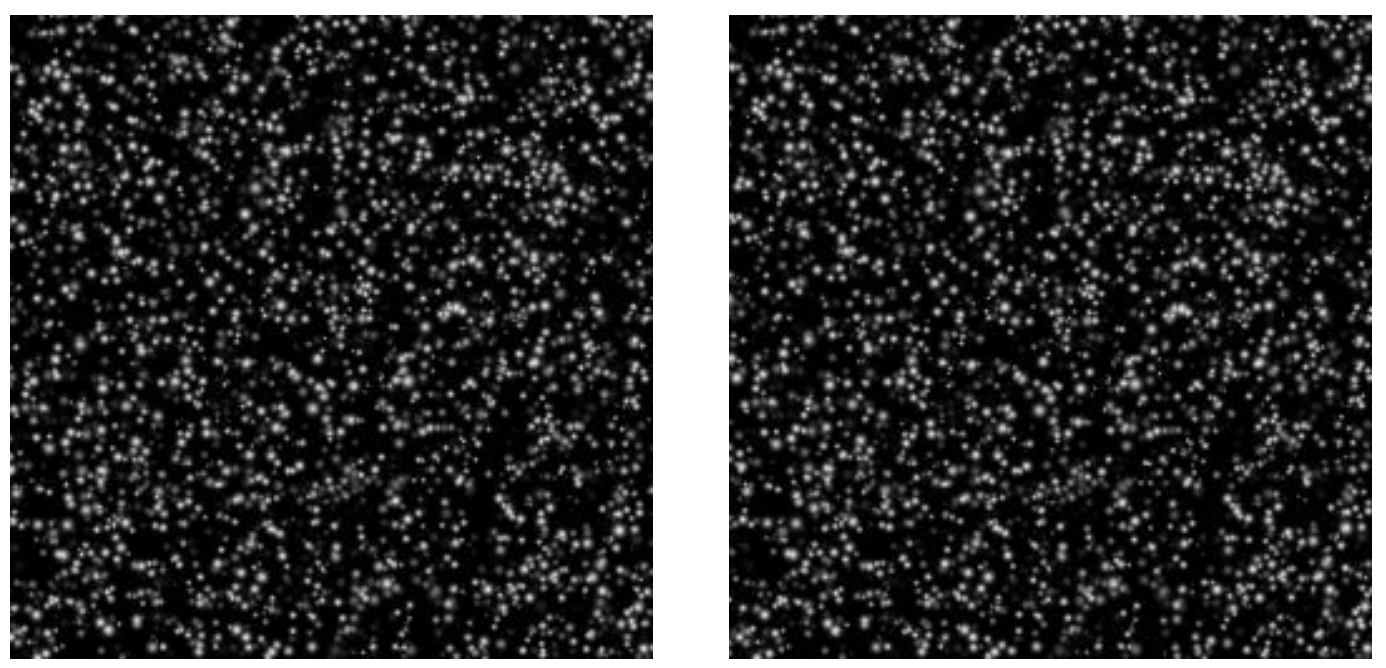

Figure 17: Small Displacement PIV Images

Using the images in Figure 17, vector plots were created. 

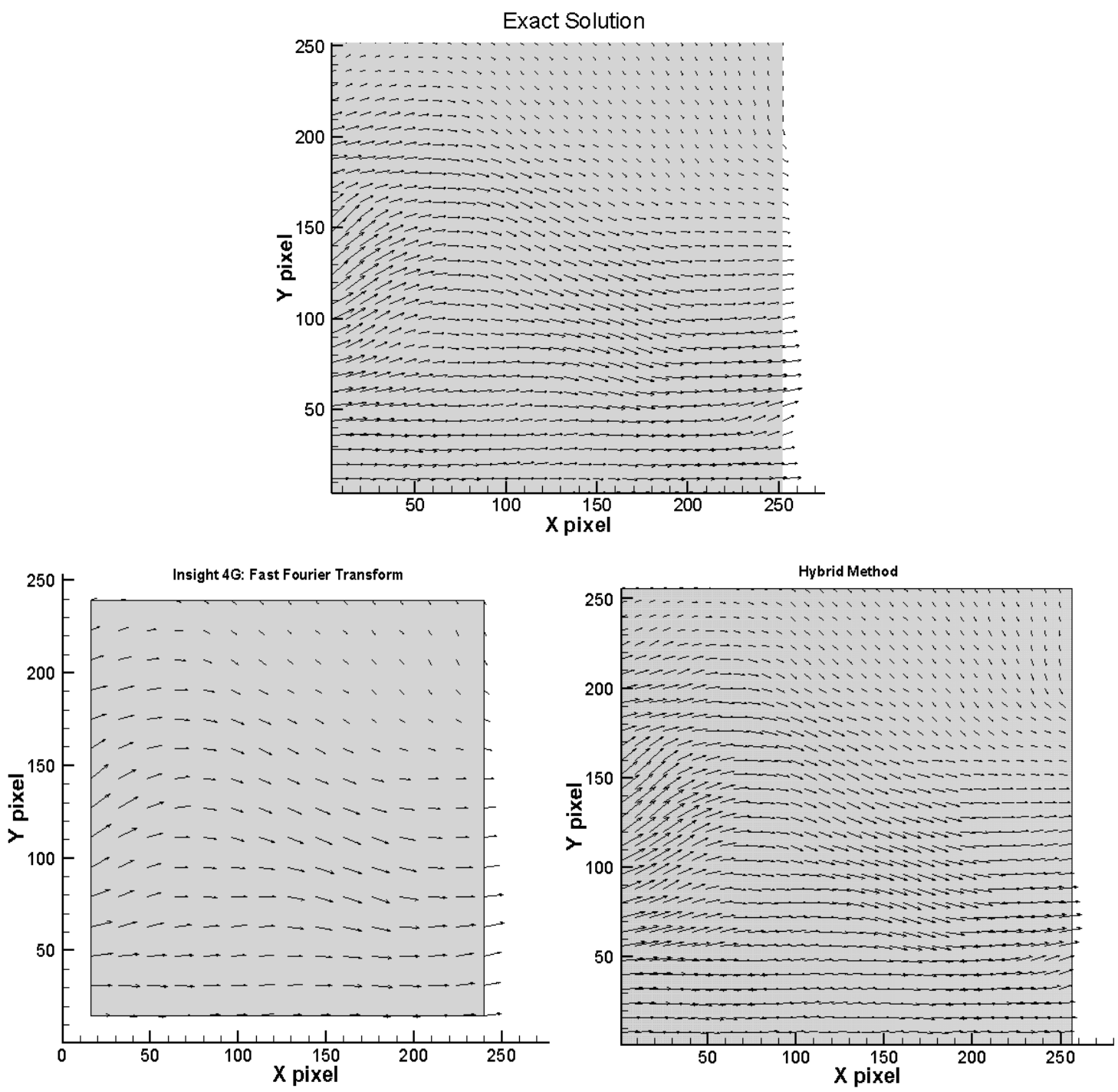

Figure 18: Vector Fields (Small Displacement)

Vectors obtained in the results are smaller in comparison to the standard images, therefore a ratio between the standard magnitude and the small displacement magnitude, given in the table of the PIV website, was created in order for the results to be comparable to the exact solution. 


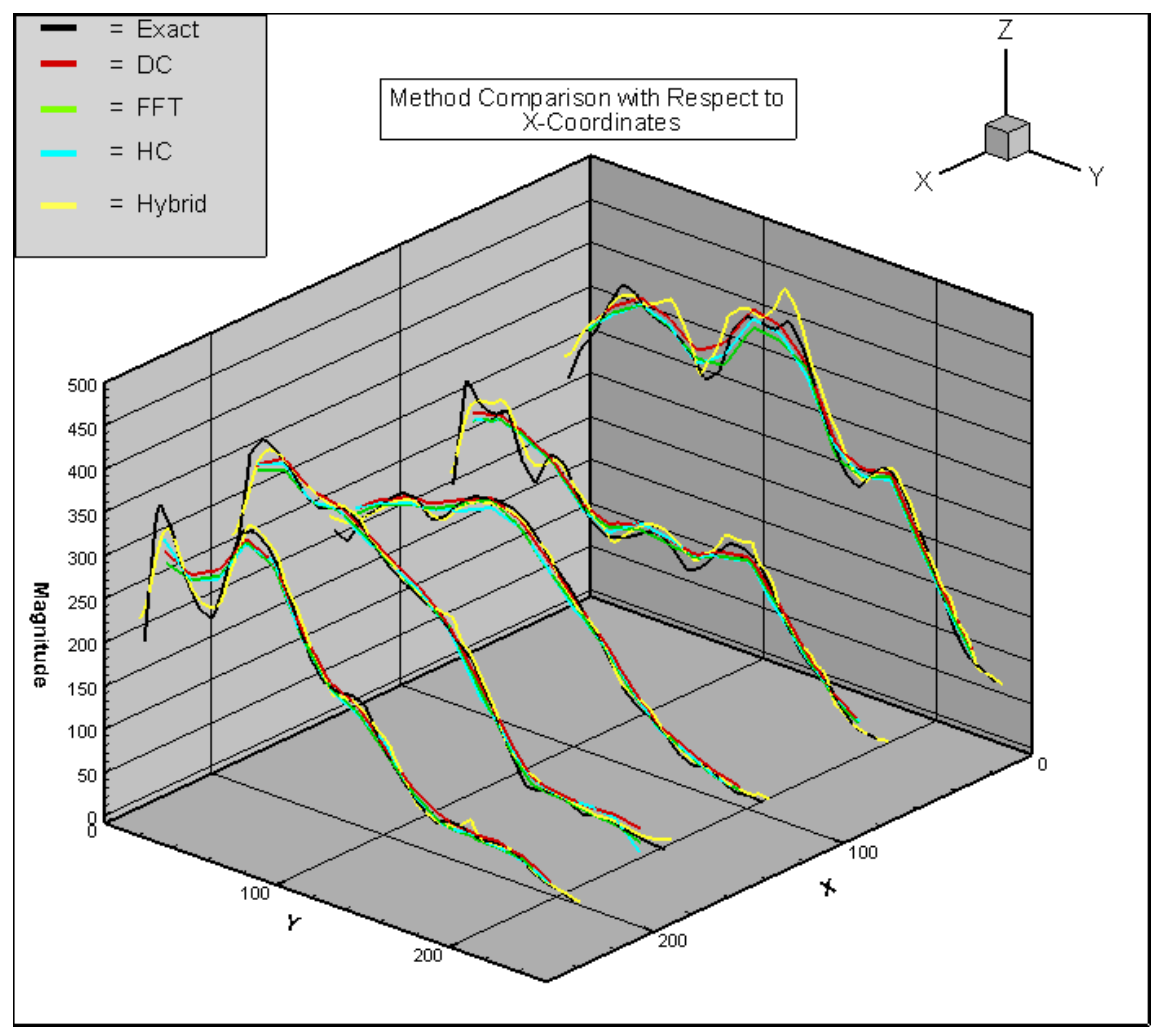

Figure 19: Method Comparison at Various X-Intercepts (Small Displacement)

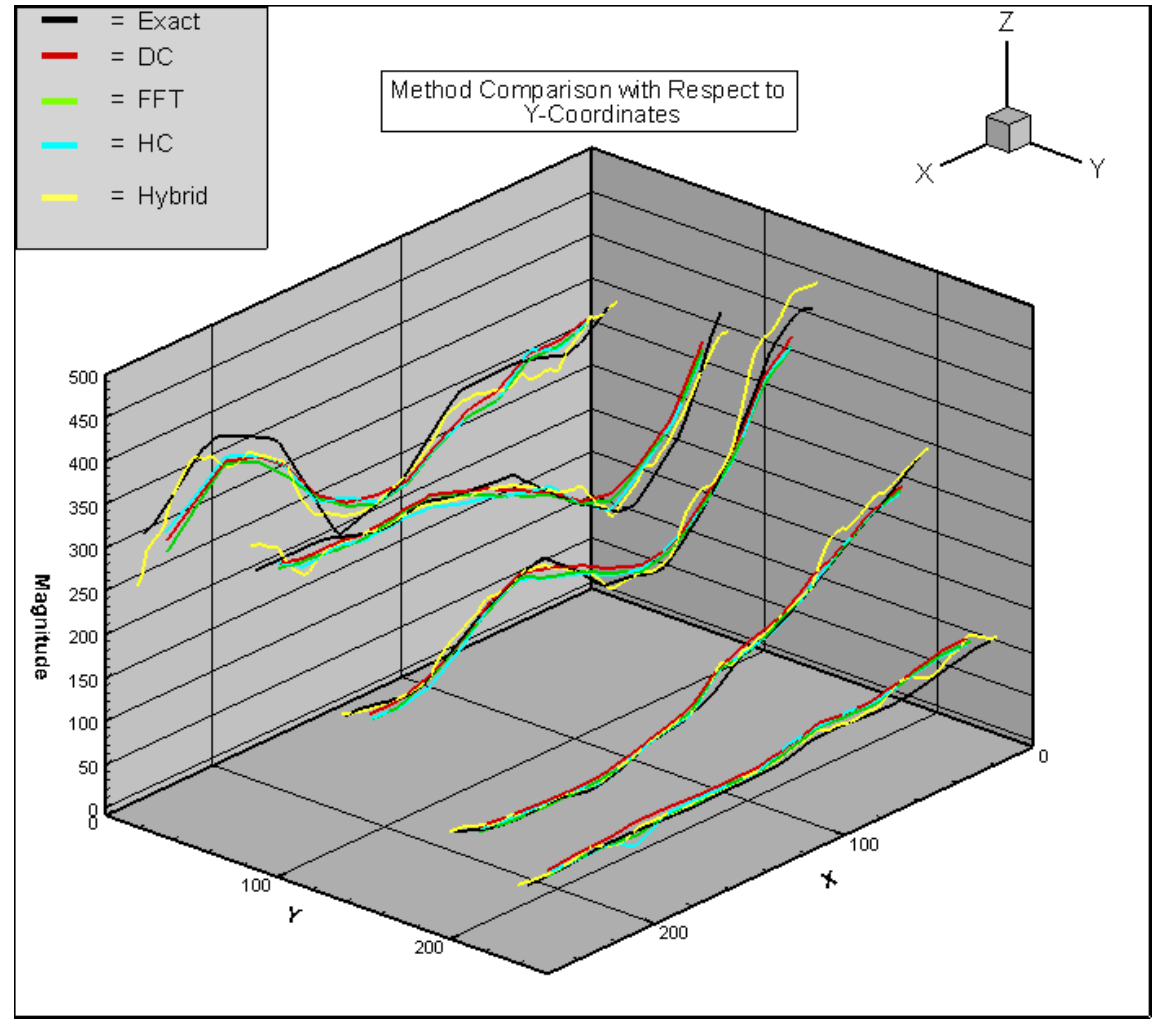

Figure 20: Method Comparison at Various Y-Intercepts (Small Displacement) 
From these results it appears that the Hybrid method is capable of following the exact solution closer than the other methods for certain regions, such as the profile for $\mathrm{x}=239$ and $\mathrm{x}=76$. Other times the Hybrid method follows suit with the other methods. To decipher which method more accurately represents the flow, error plots were created.
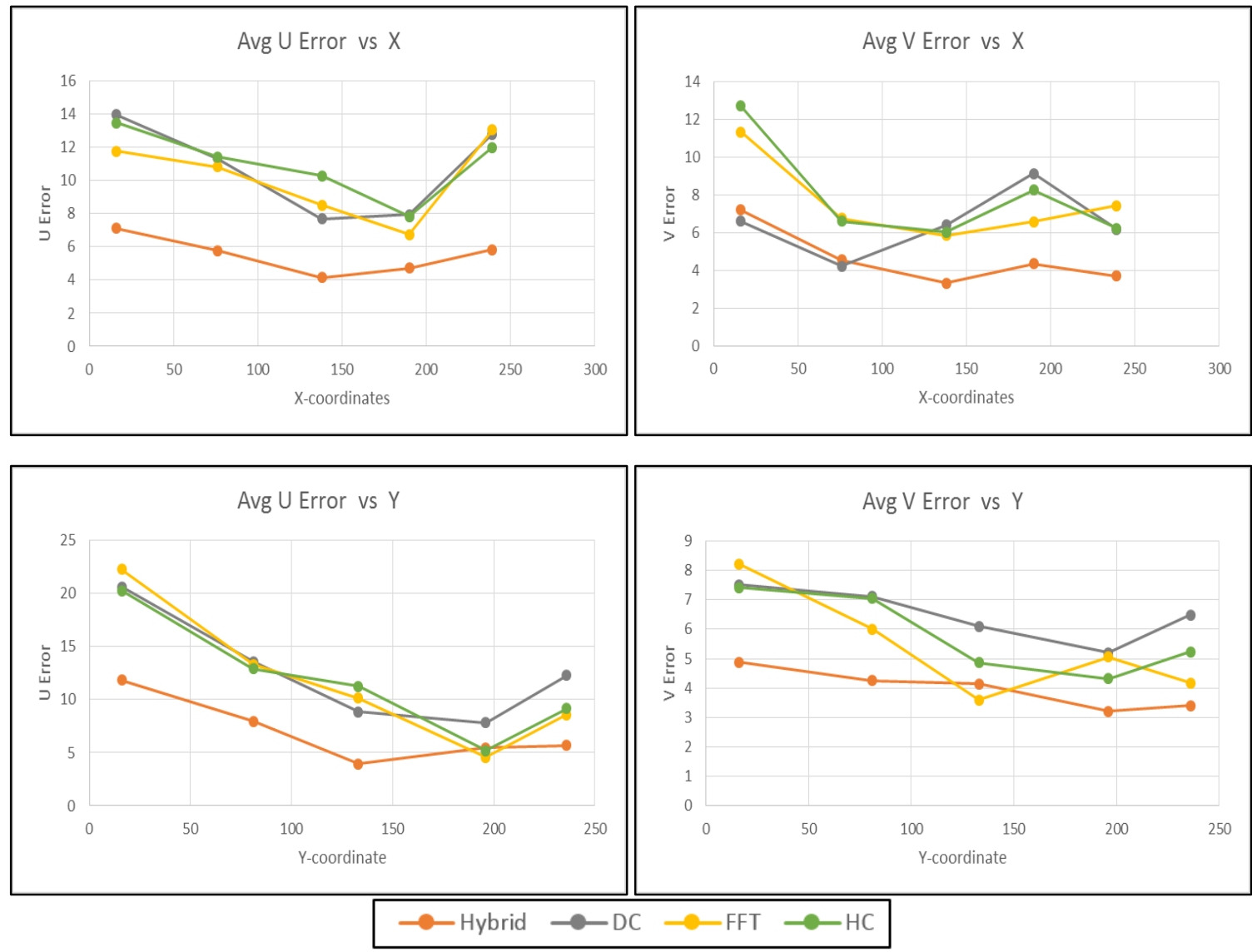

Figure 21: Average Error (Small Displacement)

Interestingly, the hybrid method outperforms all of the other methods in the U-component through the different x-coordinates. As for the V-component the hybrid method performs consistently well while the other methods get worse with the increase of the x-coordinates. For the y-coordinates, the hybrid method outperforms all of the other methods in the U- and Vcomponents with only one location, for each component, not as accurate as some of the methods. This is due to sparse particles, at certain points, as well as out-of-plane movement. Some of the particles involve differences in intensity from one image to the next, which signifies that those 
specific particles are either traveling into the plane or out of the plane. This change in intensity introduces error as explained in the optical flow methods' theoretical error section. The correlation method that performed the best was the Fast Fourier Transform, so the error was calculated with respect to each point for a 3D comparison with the hybrid method.
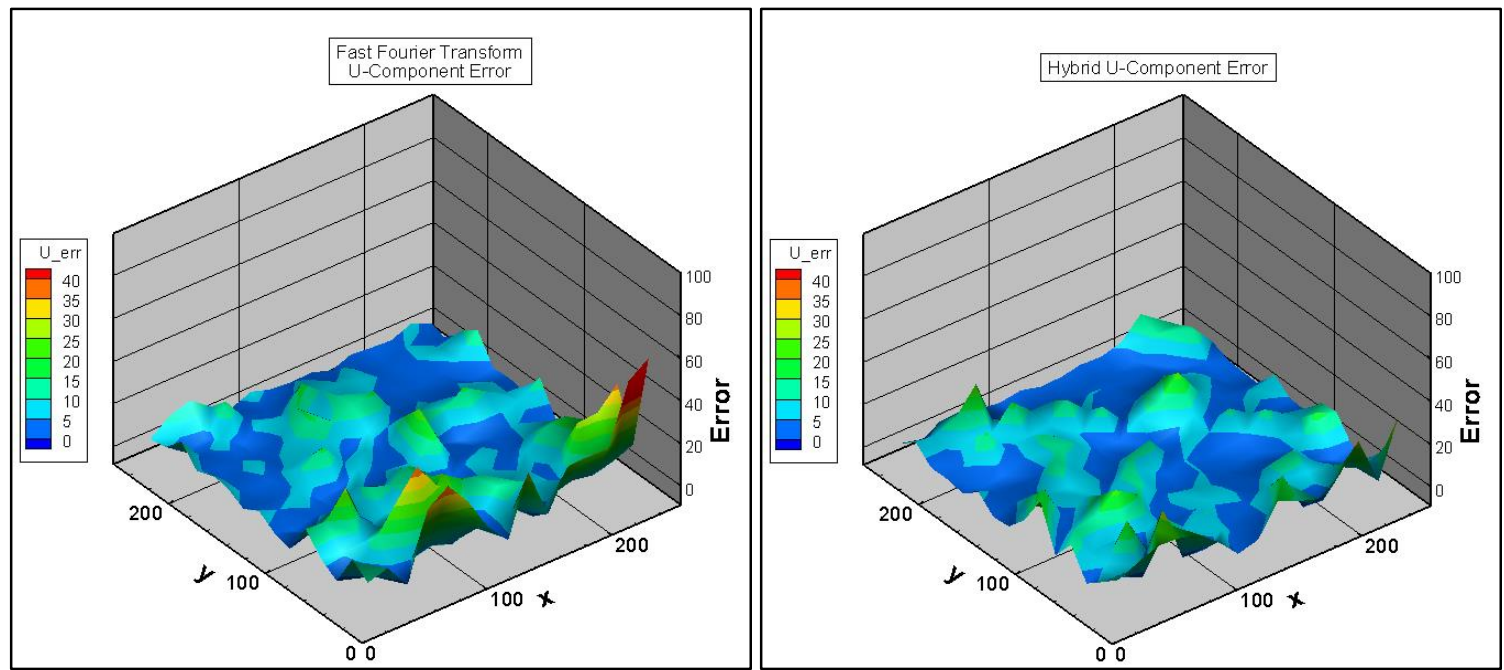

Figure 22: U-Component Error (Small Displacement)

The error from the Fast Fourier Transform is primarily located near $y=0$, where the hybrid method has sporadic points of error, but in general the amount of error in the hybrid method is less. Results of the error corresponding to the V-component are shown in Figure 23, where both methods have very similar error patterns. Hybrid method tends to calculate accurate results in the center but has a semi-ring of error around the center, where the FFT has error around and in the middle. 

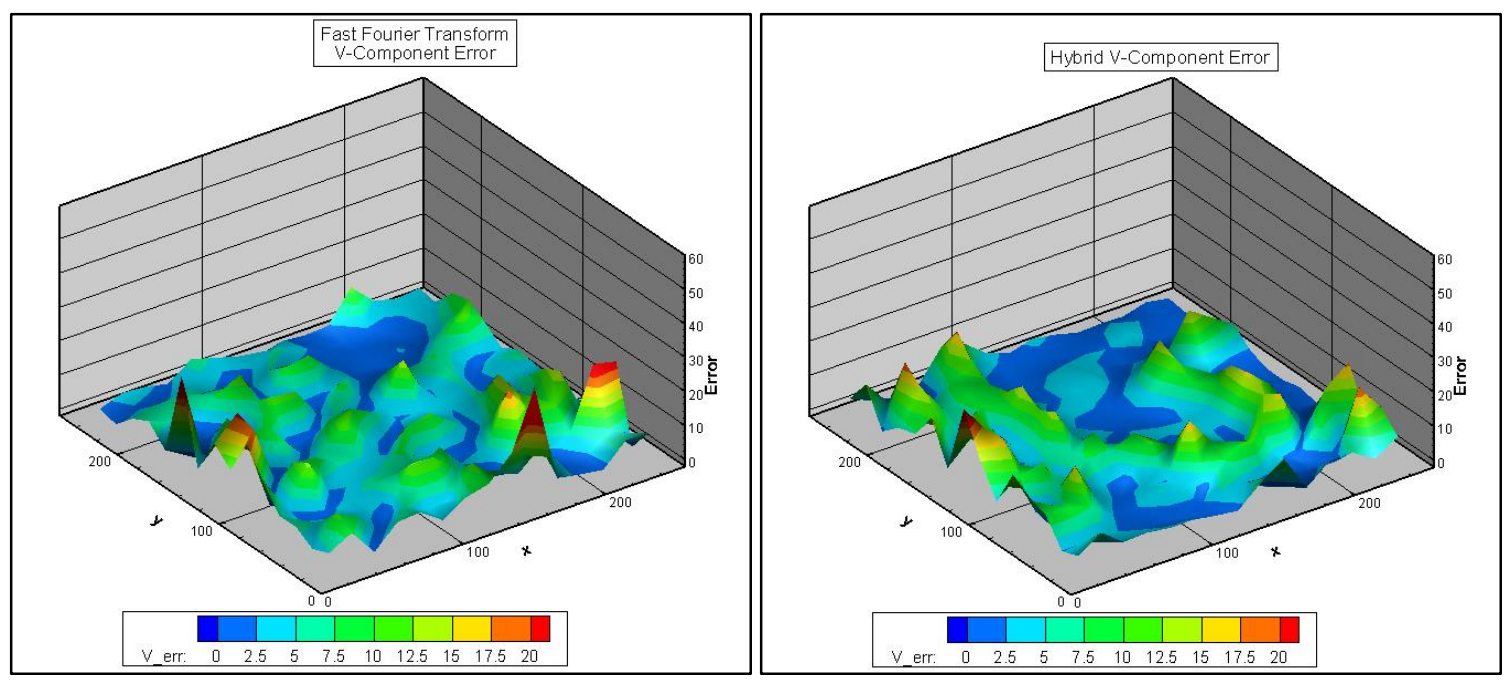

Figure 23: V-Component Error (Small Displacement)

More stimulating, though, are the results of the large displacement images because this analysis shows whether or not the Hybrid method is capable of bridging the larger displacement, with use of the Cross-correlation, to the Optical Flow method. Again these images are created through the use of increasing the magnitude of the flow with respect to the standard image, thus creating larger displacements.
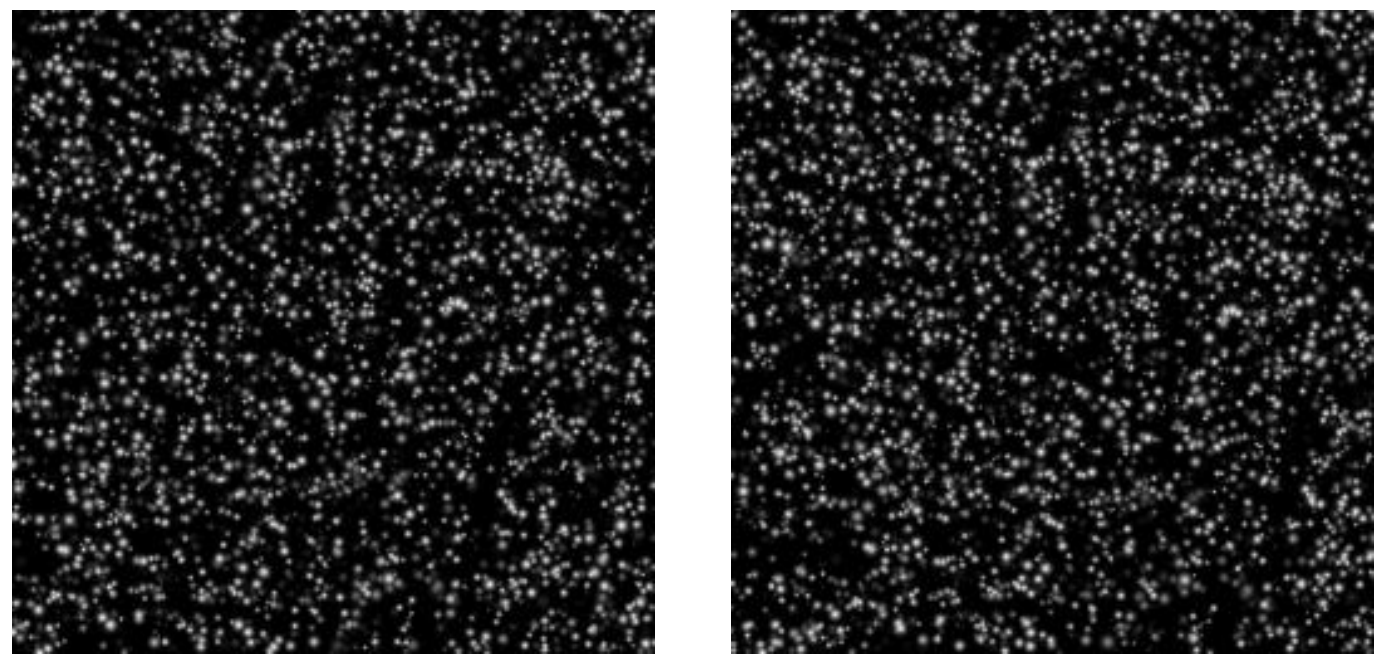

Figure 24: Large Displacement PIV Images

Identifying particle patterns, with the naked eye, from one image to the next attests to be a challenge. Therefore it was anticipated that calculating the vector field for each of the methods would be difficult for the large displacement. 

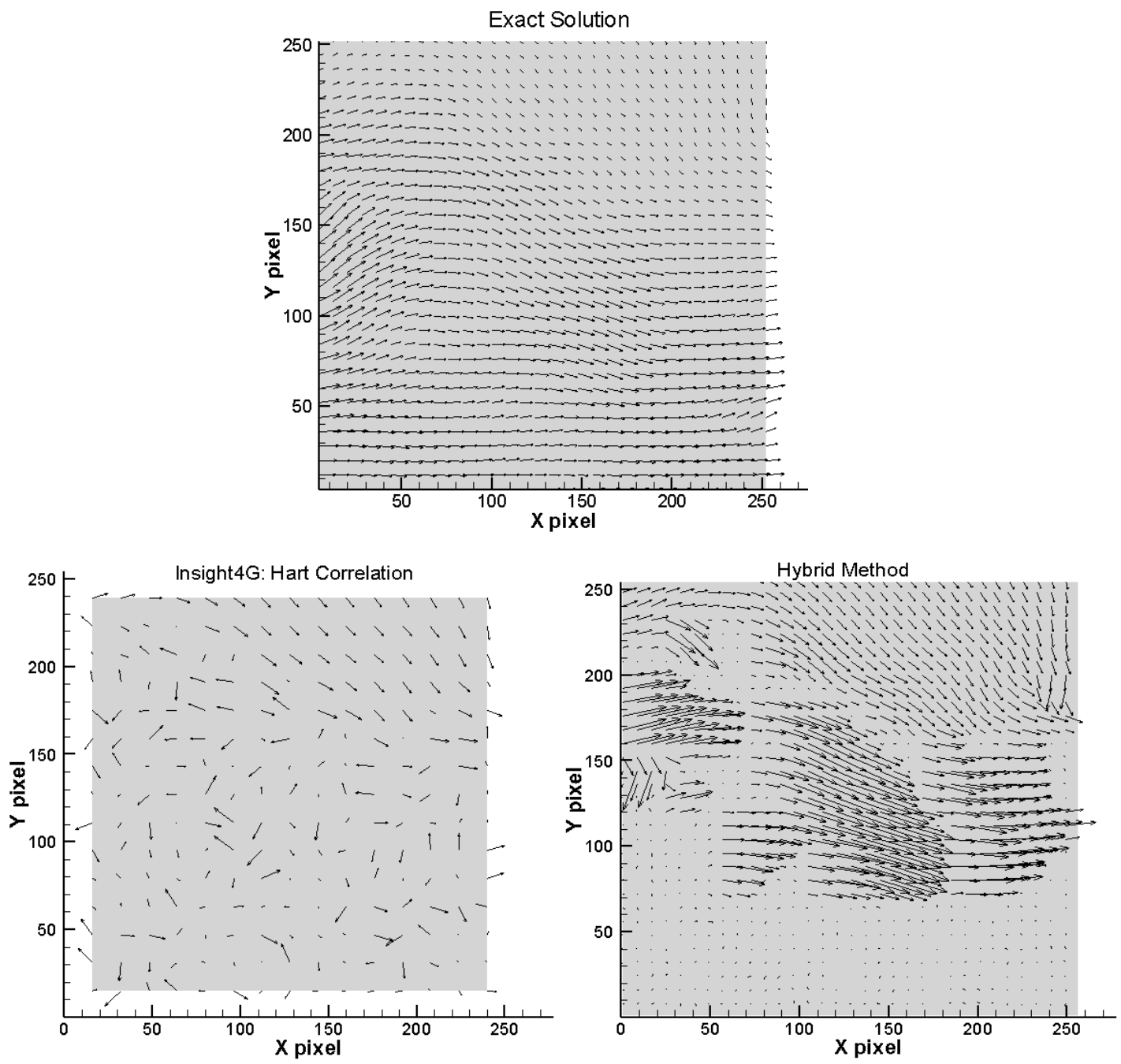

Figure 25: Vector Plots (Large Displacement)

Immediately, it is obvious that the flow near the bottom of the image plane was very difficult in calculating a correlation between the first image and the second image. Vectors in this region appear to be uncorrelated and disorganized because of the long time interval. The variation in the magnitude from the exact solution can be easily seen in Figure 26. 


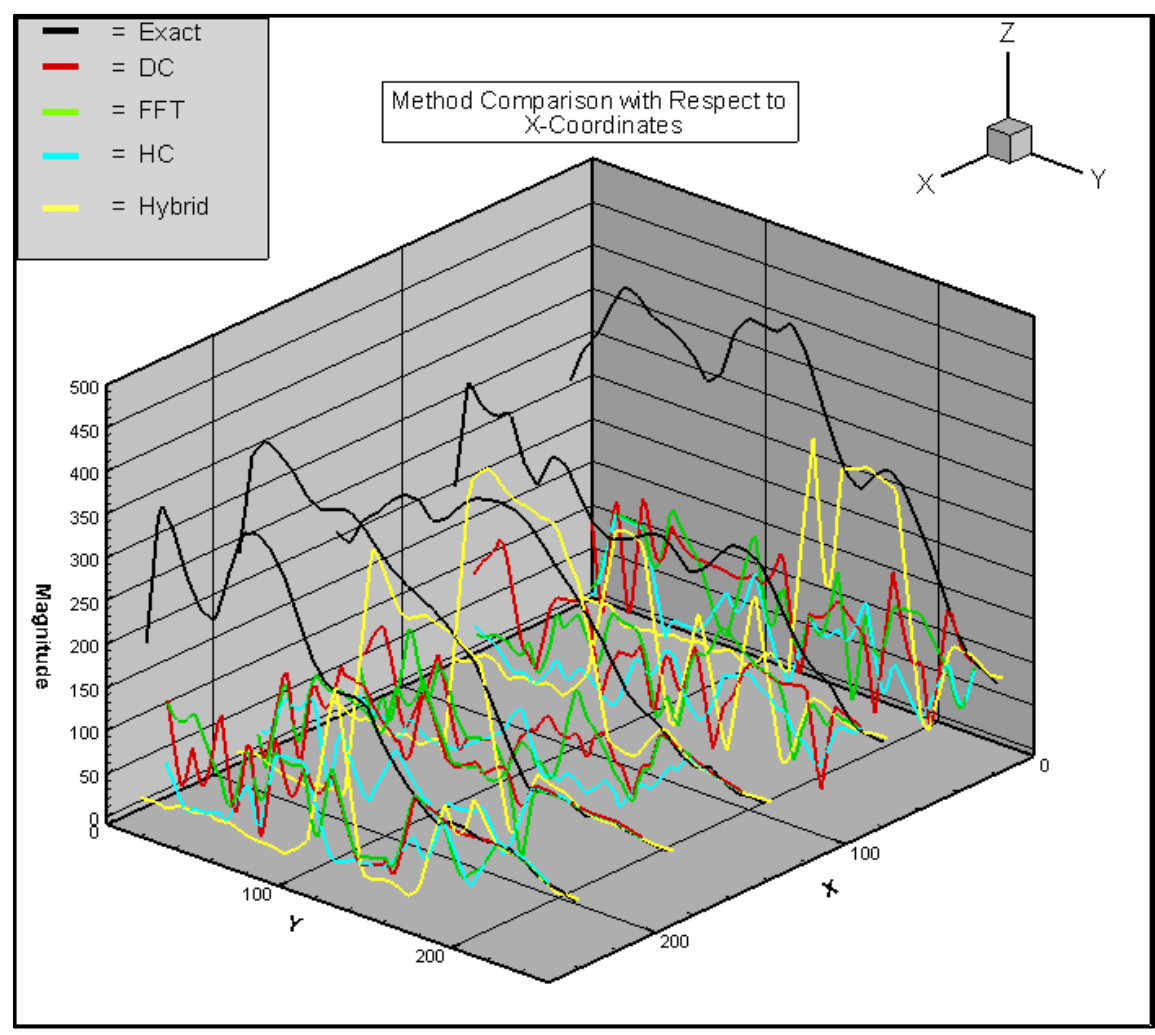

Figure 26: Method Comparison at Various X-Intercepts (Large Displacement)

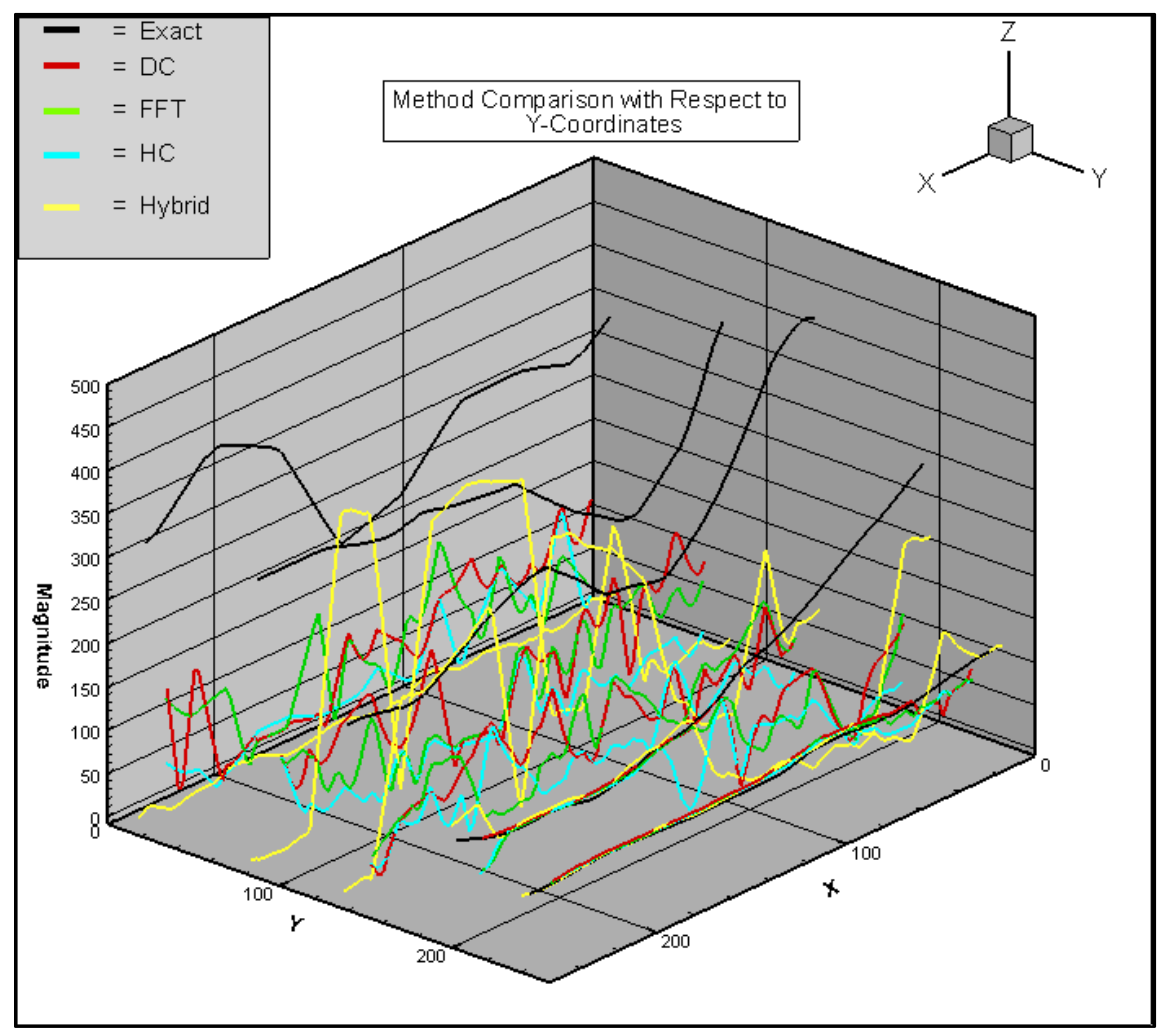

Figure 27: Method Comparison at Various Y-Intercepts (Large Displacement) 
Results show that neither of the methods truly represent the exact flow, however there are some instances where the Hybrid method does obtain similar values to the exact solution but only for a small strand of points. Figure 28 displays the average error associated with each method.
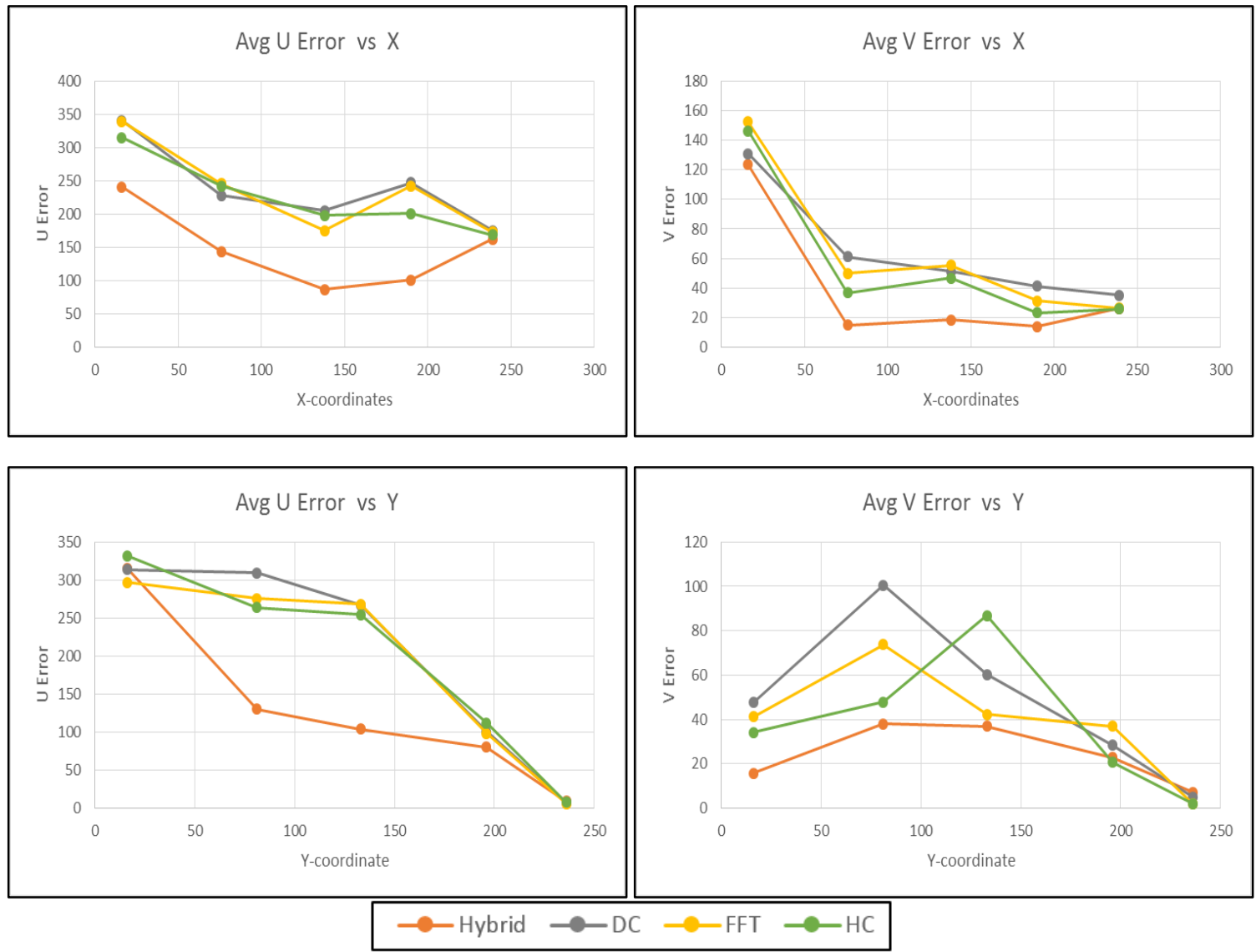

Figure 28: Average Error (Large Displacement)

It is clear that none of these methods have high accuracy when the particles have a large displacement, but the results show that the Hybrid method does have a smaller value of error, or equivalent, than the other methods associated with all four of the plots. The Hart Correlation was evaluated further by generating an error plot that corresponds to each vector's error. 

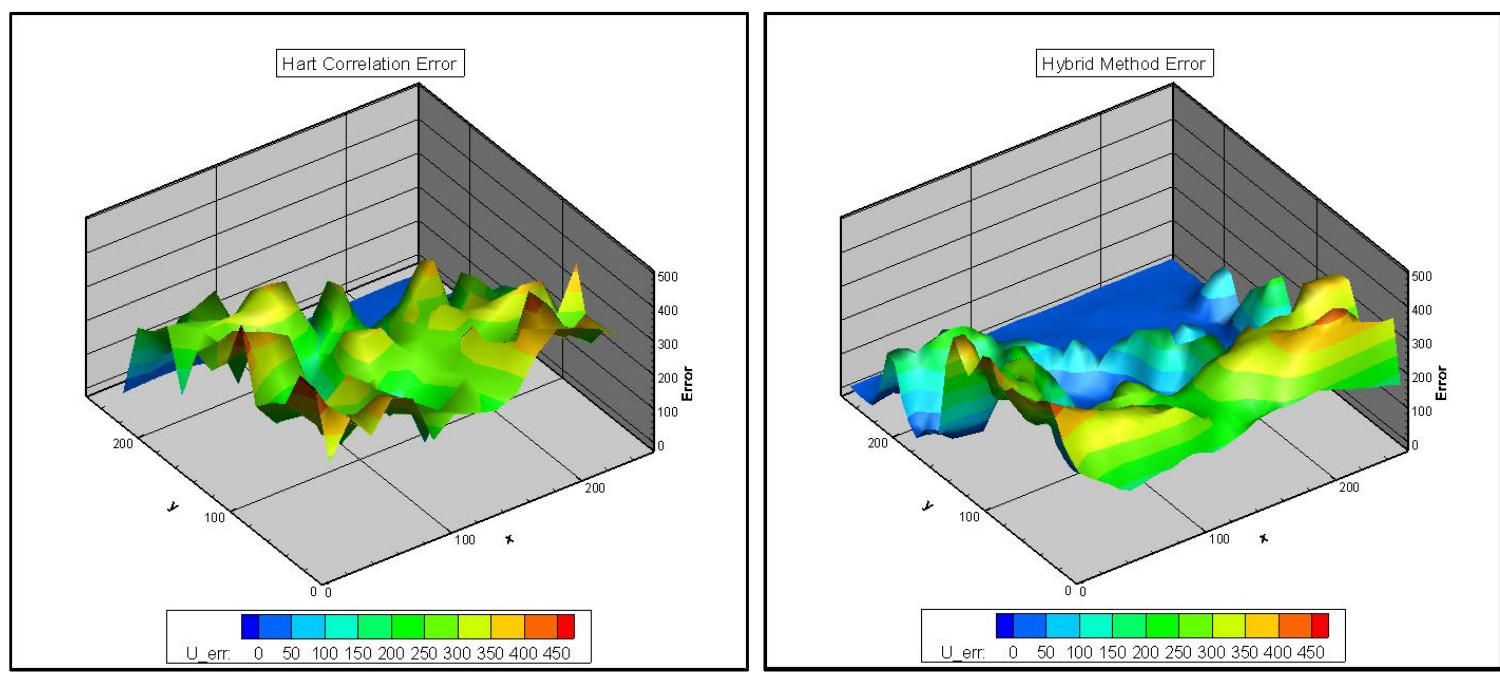

Figure 29: U-Component Error (Large Displacement)

It is clear from Figure 29 that the amount of error linked with the Hybrid method is, on average, considerably lower than the error correlated with the Hart Correlation method. Comparing the Vcomponent error also entails regions of smaller error for the Hybrid method, but both methods have a large amount of error located at the boundary of $x=16$ pixel. Only region in which both methods capture accurate results are located above $y=200$, since the flow there is considerably slower than the average flow velocity, hence smaller displacement.
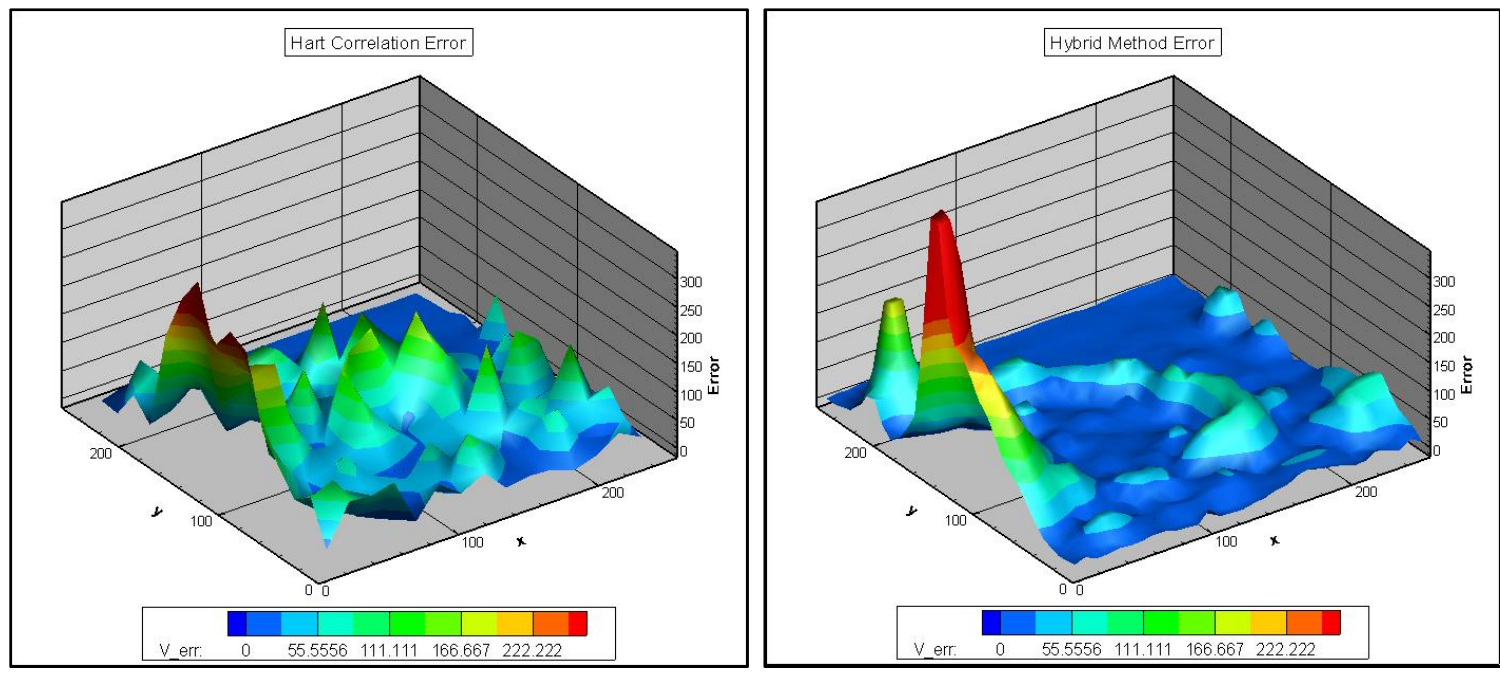

Figure 30: V-Component Error (Large Displacement) 
From these results it can be verified that large displacement images can be processed by Optical Flow when it is accompanied with Cross-correlation, thus the Hybrid method is adaptable for these situations.

\subsection{Low particle concentration}

Another problem occurs when the number of particles within the image plane are sparse. Having fewer particles means that there are fewer patterns within the flow for the methods to use for calculating the displacement.
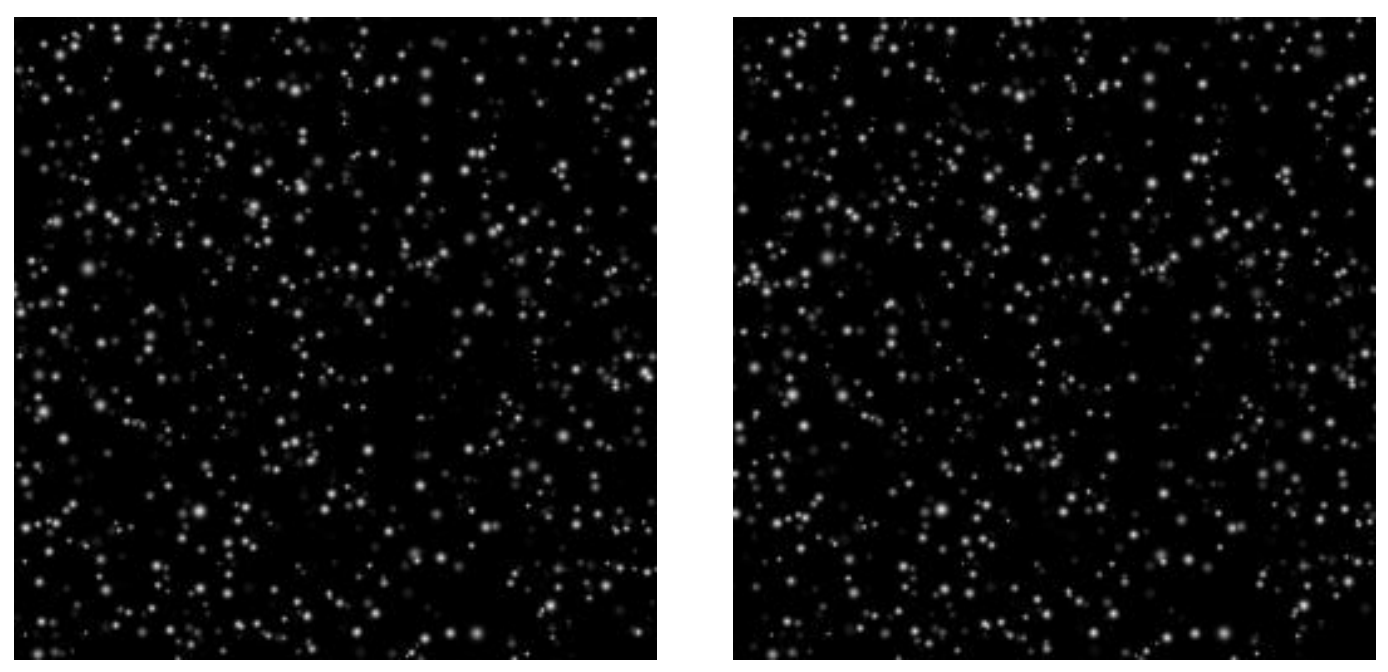

Figure 31: Low Particle Concentration PIV Images

The number of particles within these images have been reduced from the standard image but the flow field has remained unchanged. 

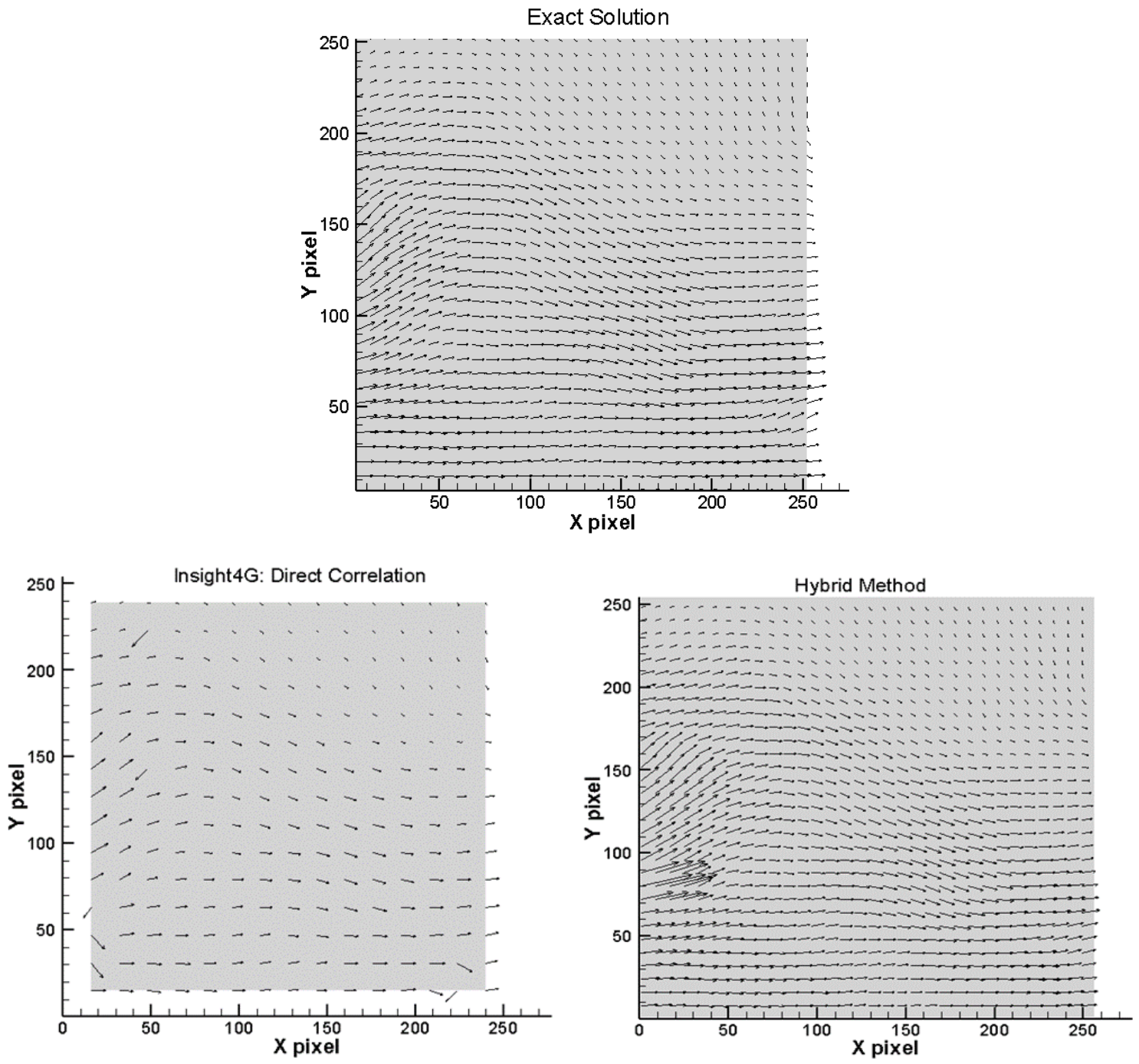

Figure 32: Vector Plots (Low Density)

Figure 32 illustrate points of interest where the vectors are unique from the other plots, especially around the boundaries of the image. 


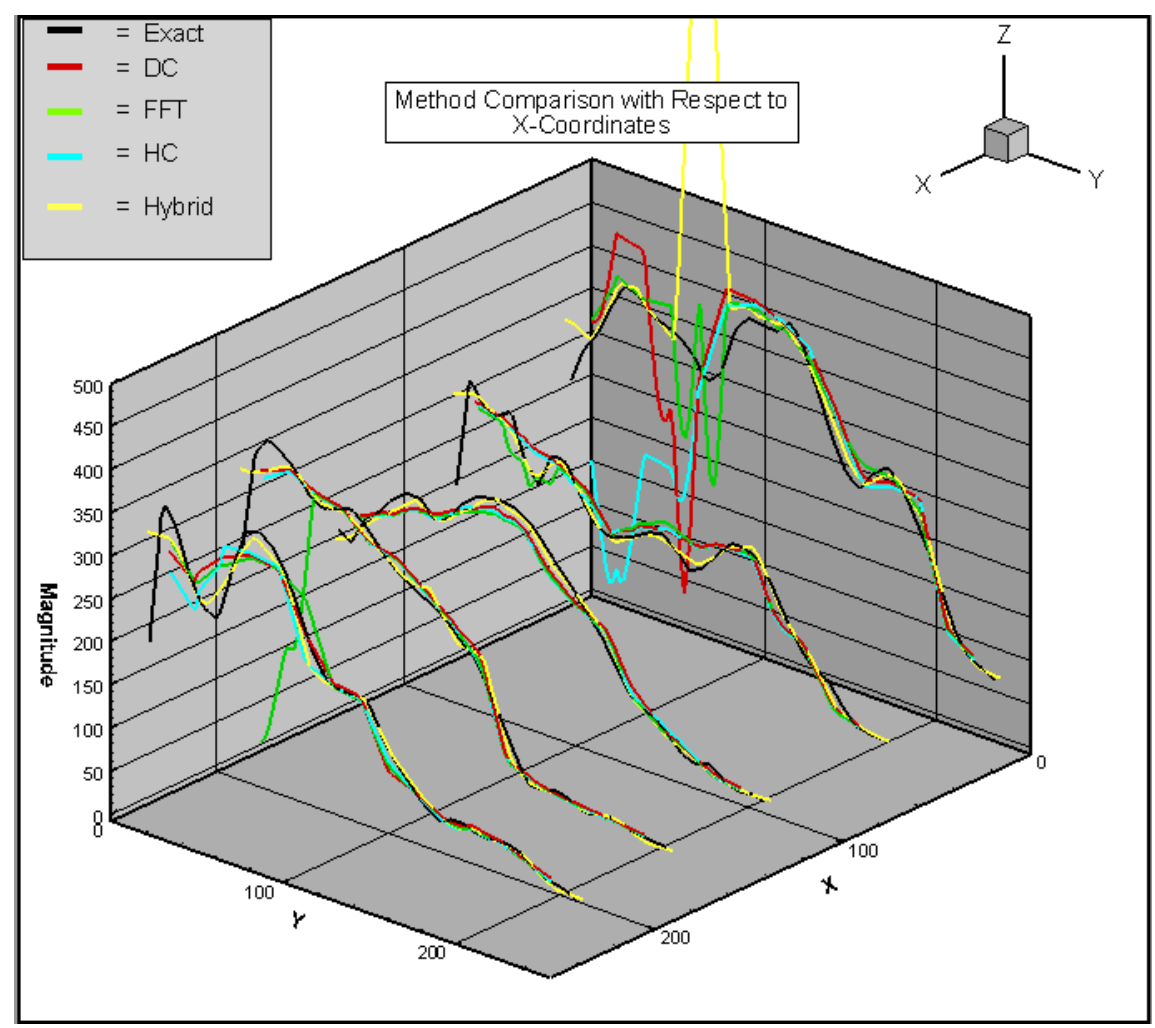

Figure 33: Method Comparison at Various X-Intercepts (Low Density)

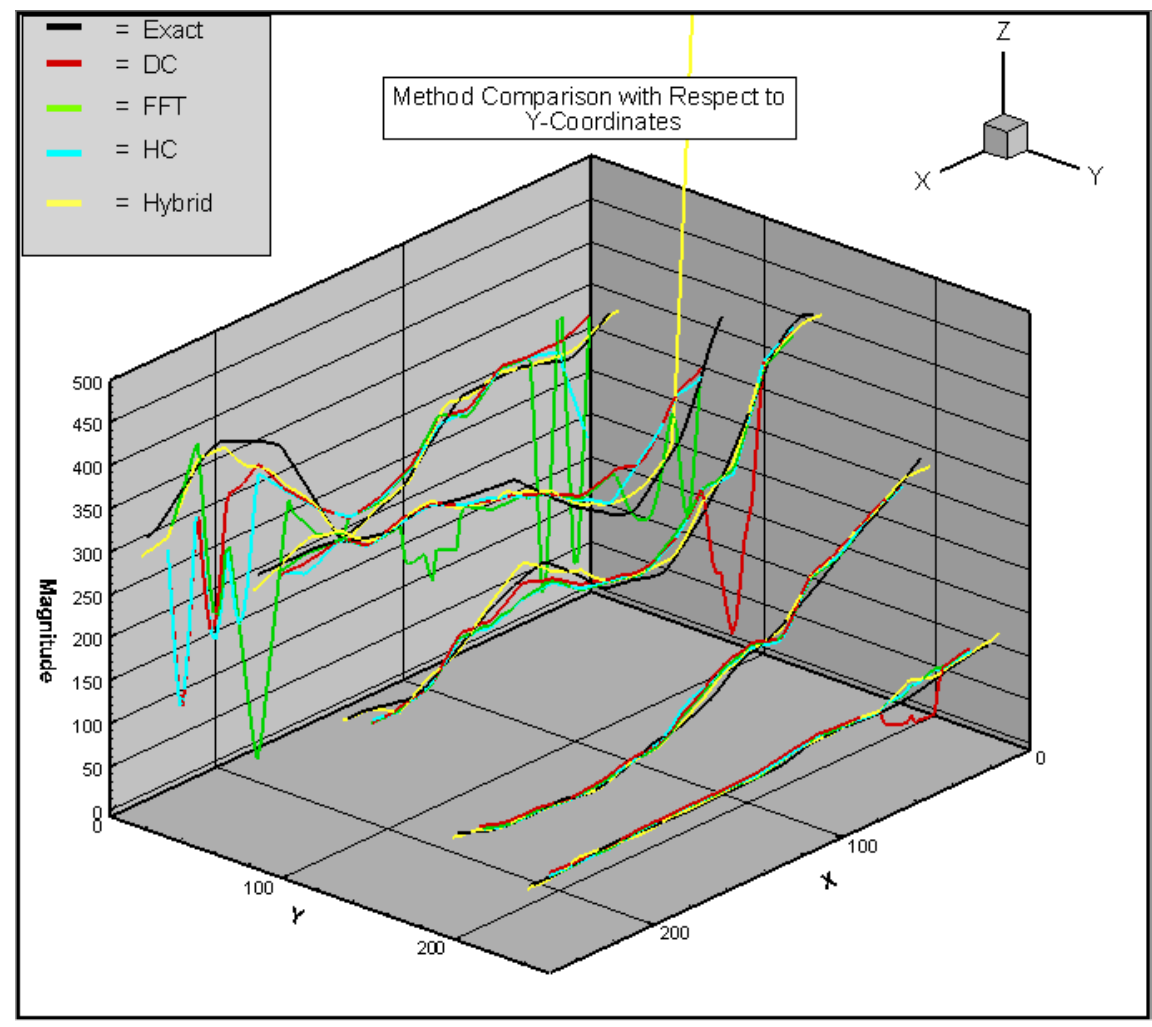

Figure 34: Method Comparison at Various Y-Intercepts (Low Density) 
There are points along $\mathrm{x}=16$ for the magnitude where each method includes a spike of variation from the exact solution. Other than that region the main deviation from intercept to intercept mainly occurs from the Fast Fourier Transform and the Direct Correlation methods. These observations are supported by the average error associated with each method in Figure 35.
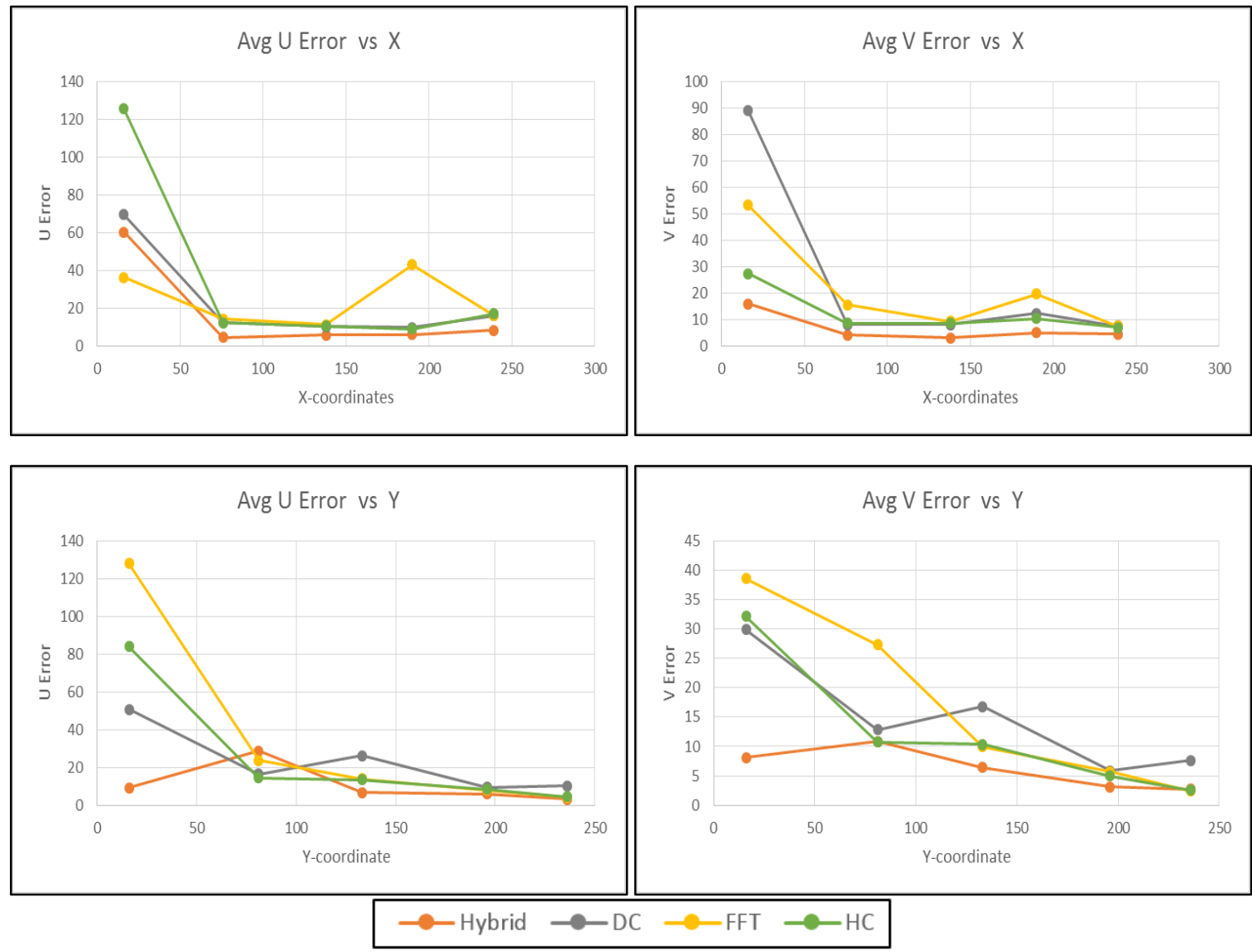

Figure 35: Average Error (Low Density)

For a more complete analysis the Direct Correlation and Hybrid method were compared using error distributions at each calculated vector. 

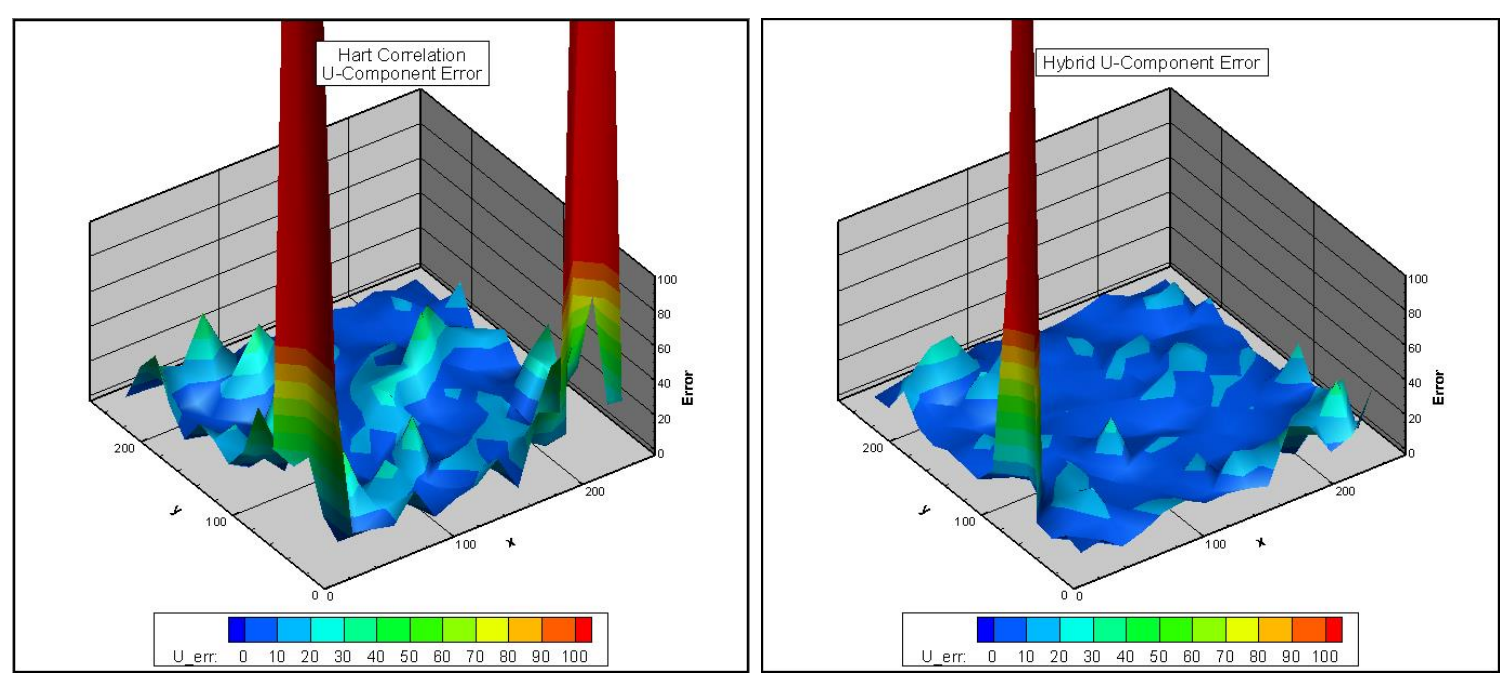

Figure 36: U-Component Error (Low Density)

Observing the amount of error related to each method in the figure above, the hybrid method tends to have a lot of error near the edge of $y=100$ but has minimal error across the rest of the domain. Whereas the Hart Correlation contains large portions of error near the boundaries, but becomes more accurate in the center similar to the hybrid method. Error related to the Vcomponent has similar differences, where the Hart correlation has large error values on the boundaries and the hybrid method has only one large value. The hybrid method was able to calculate more accurate values throughout most of the domain, especially near the boundaries.
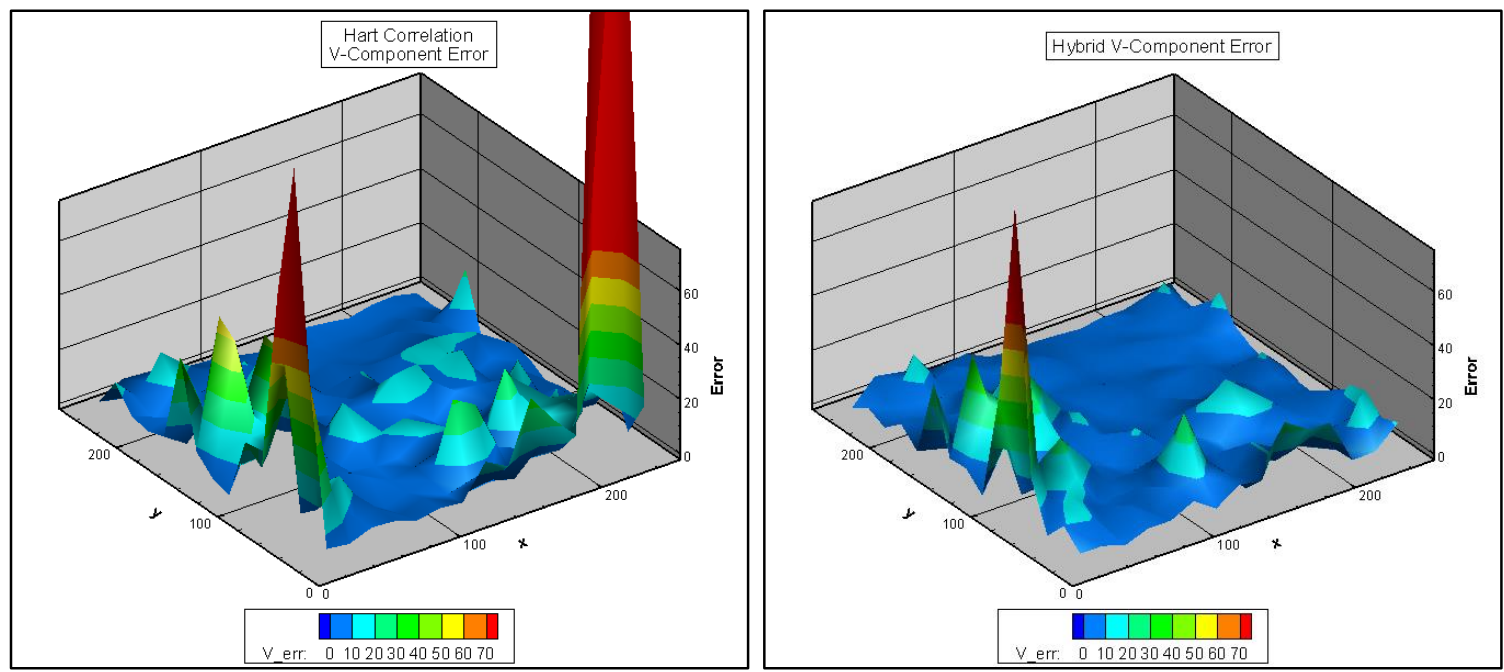

Figure 37: V-Component Error (Low Density) 


\subsection{Large particle size}

When conducting experiments there are items of equipment that are ideal, but unfortunately those pieces of equipment cannot be obtained all of the time. Hence, there are some situations in PIV where the size of the particles are very large, in comparison to standard PIV images. The following images are the test samples to illustrate what happens when the particles' size is large.
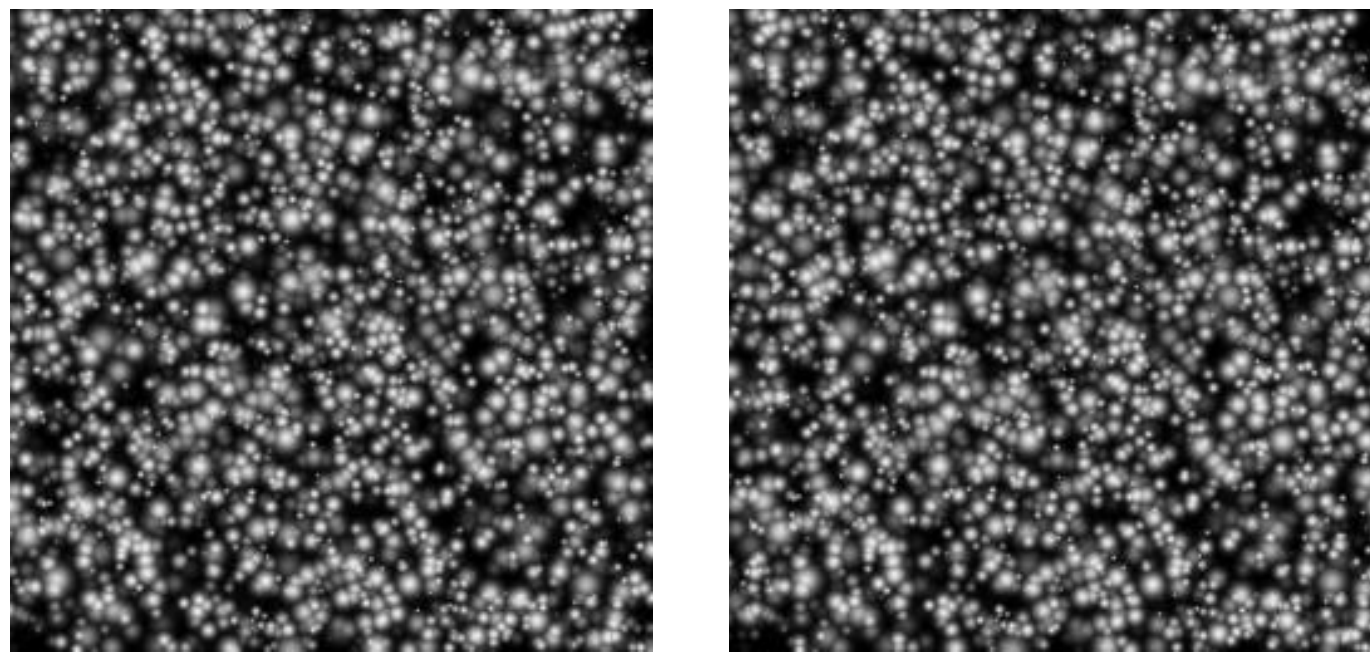

Figure 38: Large Particle Size PIV Images

Trouble occurs with the overlapping of particles which does not provide for ideal patterns within the flow, thus it is important to study which method is capable of distinguishing these patterns.

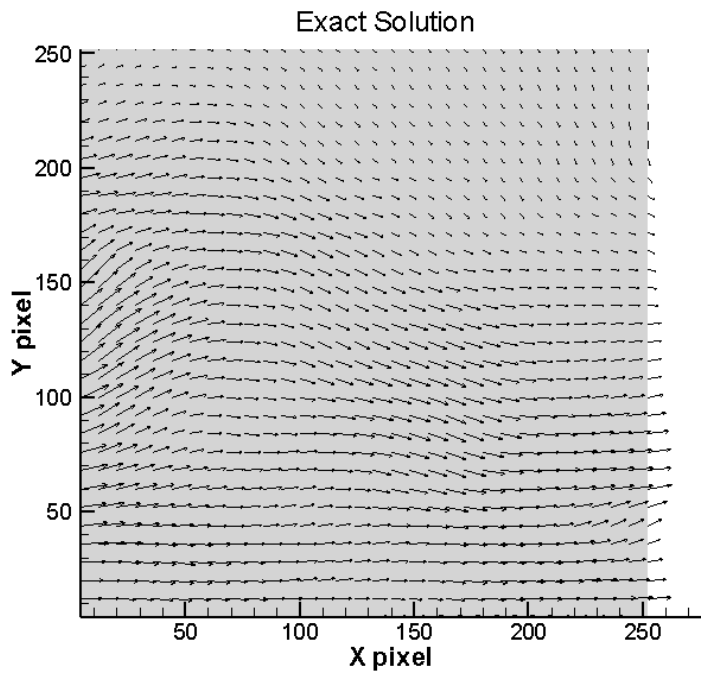



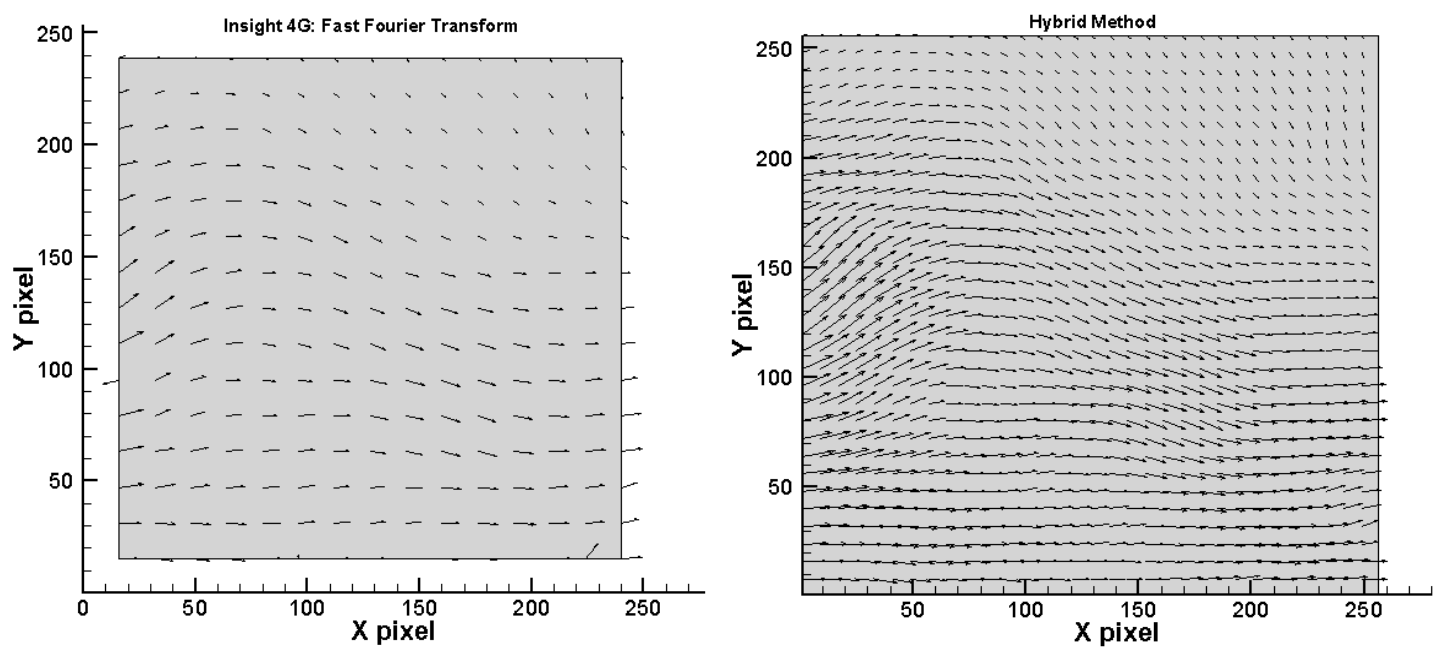

Figure 39: Vector Plots (Large Particle Size)

Immediately, erroneous vectors can be identified near the boundary of the Fast Fourier

Transform, on the left side. Figure 40 and Figure 41 displays the more subtle errors each method contains.

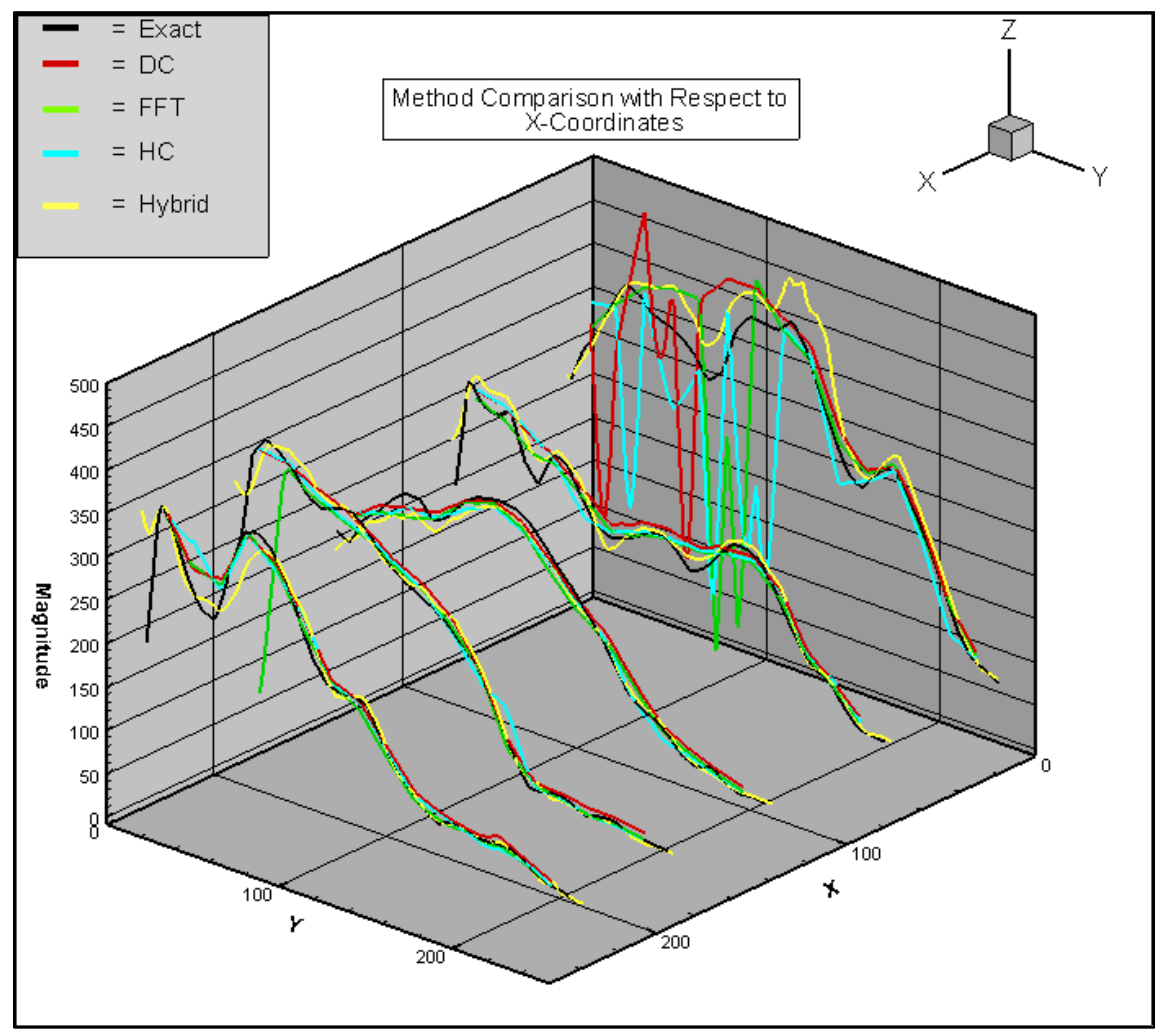

Figure 40: Method Comparison at Various X-Intercepts (Large Particle Size) 


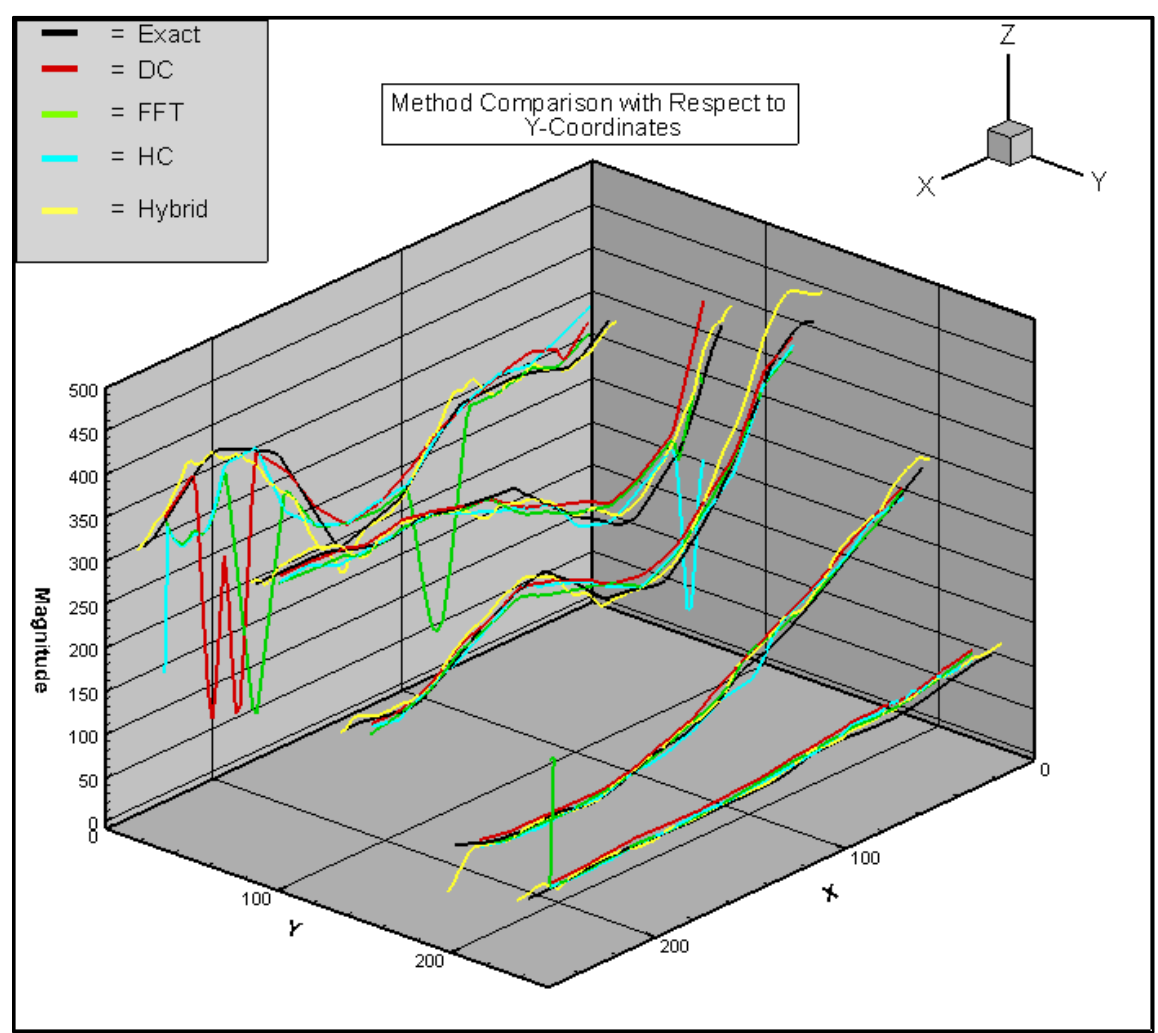

Figure 41: Method Comparison at Various Y-Intercepts (Large Particle Size)

By noticing $x=16$ pixel in Figure 40, the Hybrid method performs extremely well, with respect to the other methods. Observing the other methods, they tend to lack the fine details along the curve of the solution whereas the Hybrid method is able to pick up on these slight velocity changes. This is also obvious for the different y-intercepts in Figure 41. The error plots below give more insight into the accuracy of each method.
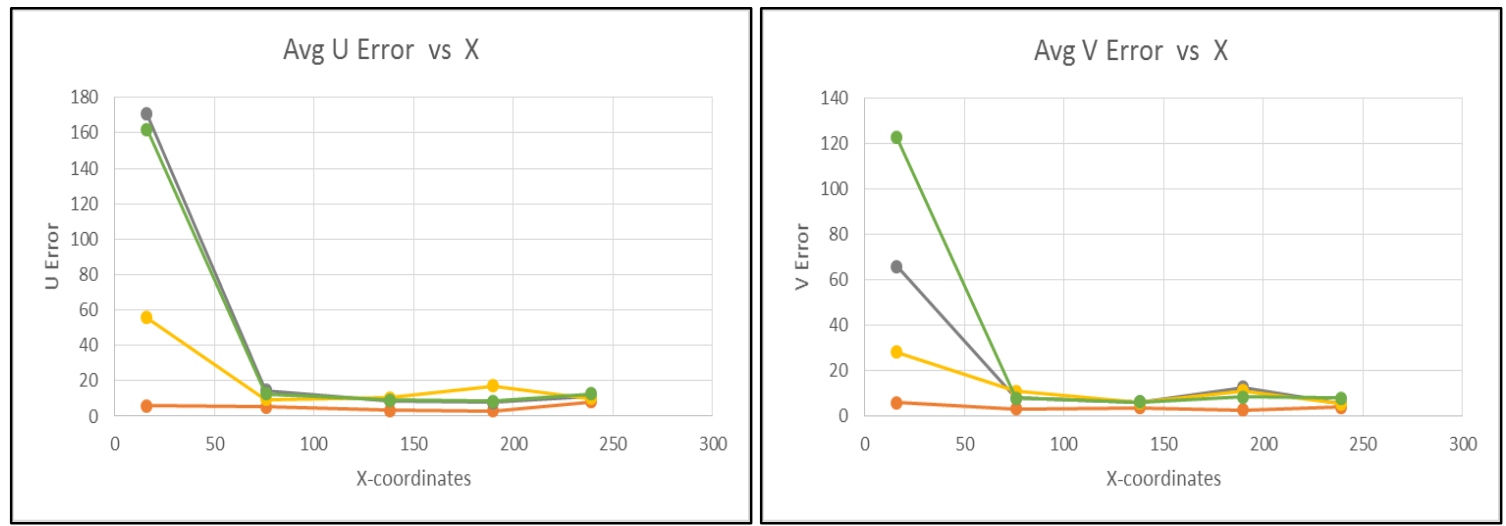

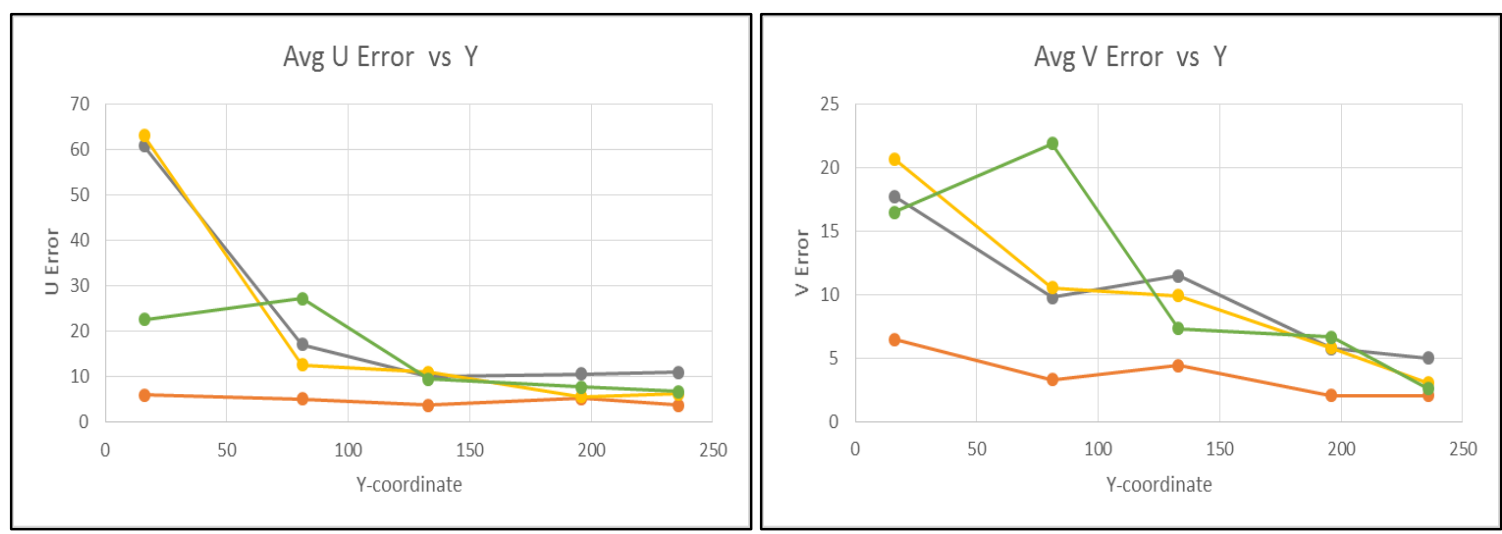

Figure 42: Average Error (Large Particle Size)

Considering the error associated with the $\mathrm{U}$ - and V-components for the $x$-coordinates, the hybrid method is consistent and comparable, if not better, than the other methods. The same can be described about the error in the U-component for the $y$-coordinates. Problems in calculating the V-component through the $y$-coordinates are evident for the correlation methods, but the hybrid method's error remains consistently small. One common observation throughout all four of the plots is that the error associated with the hybrid method at the boundaries are almost always better than the other methods. These error plots show the Fast Fourier Transform to be the better correlation method for these types of images, therefore comparisons will be extended with this method.
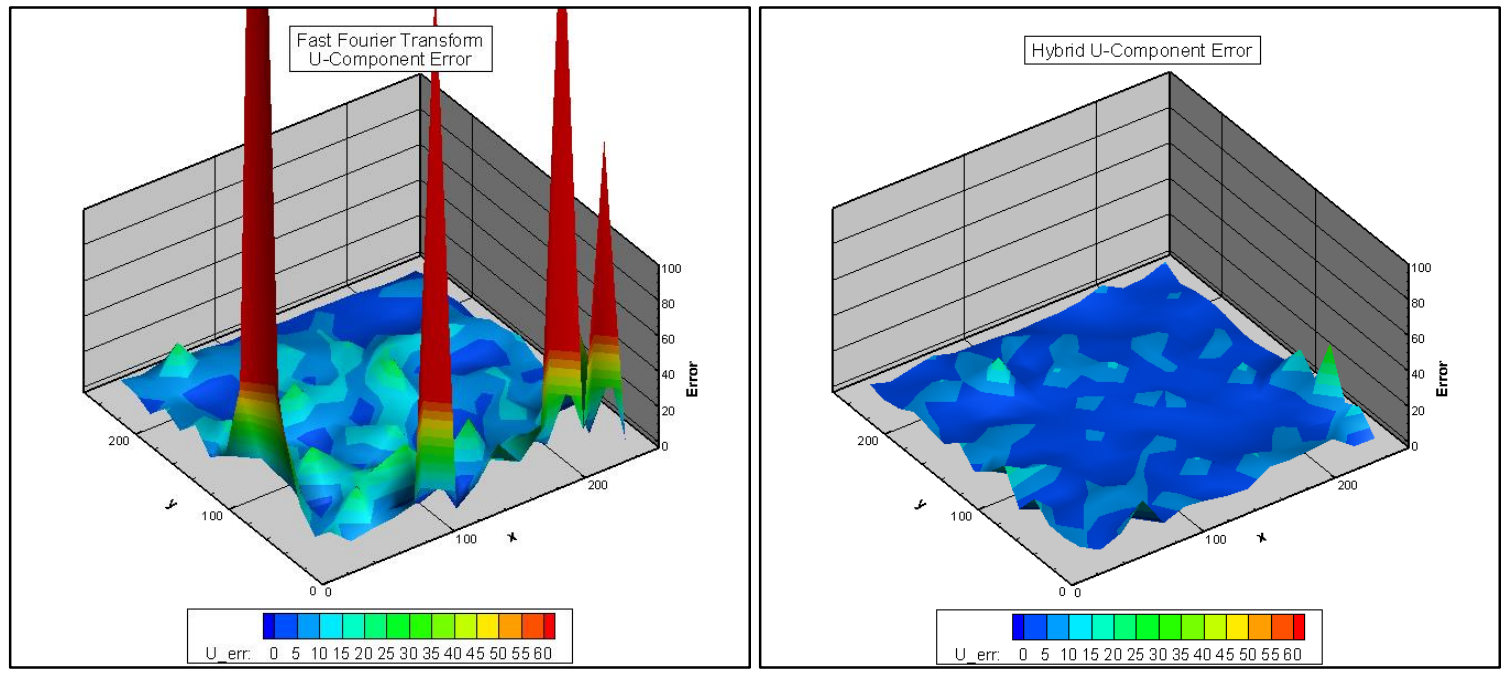

Figure 43: U-Component Error (Large Particle Size) 
It is clear that the Fast Fourier Transform method incorporated a vast amount of error. On the other hand the hybrid method had significantly less error, especially near the boundaries. As for the V-component error in Figure 44, the hybrid method doesn't have nearly any error with respect to the error associated with the Fast Fourier Transform. Boundary error is self-evident for the FFT, while the hybrid method's error is very seldom.
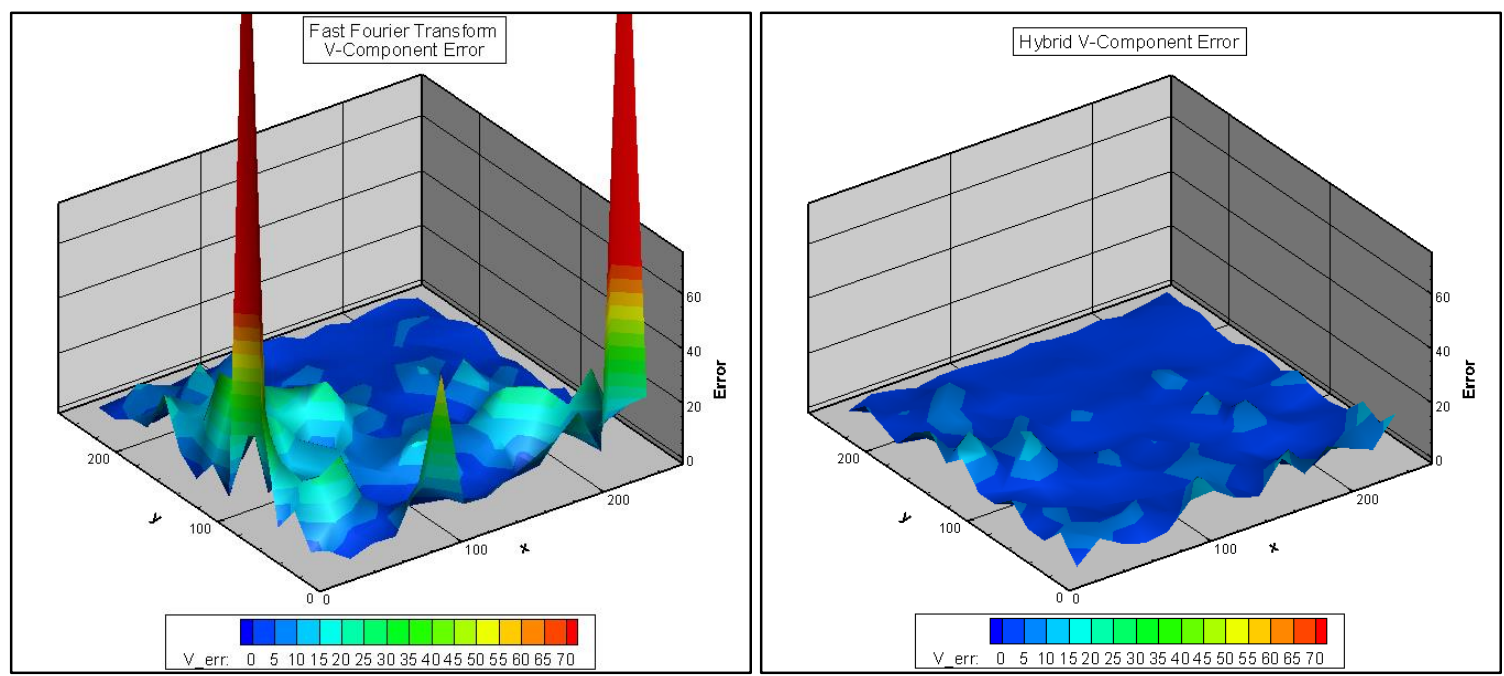

Figure 44: V-Component Error (Large Particle Size)

\section{Conclusion}

The need for a hybrid method, with regards to PIV, is evident. Liu and Shen (2008) have already hinted that the combination of a correlation method with the Optical Flow method could be promising. Confirming the validity of the hybrid method was accomplished by assessing five parameters encountered in PIV imaging and comparing the results obtained by the hybrid method with other correlation techniques. First type of PIV imaging was the standard image in which the hybrid method was generally more accurate across the image but significantly better near the boundaries. For images with low particle density, the hybrid method is comparable to the other methods but it is clear that the average error values are larger than the standard error plots. Similar to the results from the intensity gradient analysis, low density images provide a challenge for the optical flow method. When the particles possess large displacements between images, 
none of the methods have great accuracy and therefore neither of the methods are practical for this specific situation. Hybrid method excels when evaluating images with large particles, whereas the other methods incorporate a significant amount of error. Also, regions near the boundaries, for both the U- and V-components, are calculated especially well for the hybrid method in comparison with the other methods. Intensity gradient values, for the large particle size after filtering, are comparative to the characterized intensity gradient of 13.4 from the intensity gradient analysis. Again the hybrid method can calculate the flow more accurately than the other methods when the displacement of the particles are small. This case demonstrates the accuracy of the optical flow method over the sub-pixel method that the other correlation methods utilized. Overall, the hybrid method is well suited for calculating the boundaries of the image and within the middle region. Interesting, is that the hybrid method performs exceptionally well in the large particle sized images which suggests that the method is able to identify the flow more accurately when the intensity is continuous. Another point is that the other methods do not acquire the same accuracy for small displacements, as the hybrid method, due to the sub-pixel calculations that are not as precise as the Optical flow method. 


\section{References}

Adrian, R. J. 2005 Twenty years of particle image velocimetry. Experiments in Fluids 39, 159169.

Barbu, V., \& Marinoschi, G. 2016 An optimal control approach to the optical flow problem. Systems \& Control Letters 87, 1-9.

Bastiaans, R. J. M. 2000 Cross-correlation PIV; theory, implementation and accuracy. Eindhoven University of Technology, Eindhoven, Netherlands.

Chen, X., Zillé, P., Shao, L., Corpetti, T. Optical flow for incompressible turbulence motion estimation. Experiments in fluids, 2015, to be published. <10.1007/s00348-014-1874-6>. $<$ hal-01102944>

Corpetti, T., Heitz, D., Arroyo, G., Mèmin, E., \& Santa-Cruz, A. 2005 Fluid experimental flow estimation based on an optical-flow scheme. Experiments in Fluids.

Hart, D. P. 1999 Super-Resolution PIV by Recursive Local-Correlation. Journal of Visualization, Vol. 10.

Hèas, P., Mèmin, E., Papadakis, N., \& Szantai, A. 2007 Layered Estimation of Atmospheric Mesoscale Dynamics From Satellite Imagery. IEEE Transactions on Geoscience and Remote Sensing, Vol. 45, No. 12.

Heitz, D., Mèmin, E., \& Schnörr, C. 2010 Variational fluid flow measurements form image sequences: synopsis and perspectives. Exp. Fluids 48, 369-393.

Horn, B. K.P., \& Schunck, B. G. 1981 Determining Optical Flow. Artificial Intelligence 17, 185203.

Hu, H., Saga, T., Kobayashi, T., Okamoto, K., \& Taniguchi, N. 1998 Evaluation of the Cross Correlation Method by Using PIV Standard Images. Journal of Visualization, Vol. 1, No. $1,87-94$

Hurlburt, N., \& Jaffey, S. 2015 A spectral optical flow method for determining velocities from digital imagery. Earth Science Informatics 8: 959-965.

Keane, R. D., \& Adrian, R. J. 1992 Theory of cross-correlation analysis of PIV images. Applied Scientific Research 49, 191-215.

Liu, T., Merat, A., Makhmalbaf, M. H. M., Fajardo, C., \& Merati, P. 2015 Comparison between optical flow and cross-correlation methods for extraction of velocity fields from particle images. Experiments in Fluids 56: 166.

Liu, T., \& Shen, L., 2008 Fluid flow and optical flow. Journal Fluid Mech., Vol. 614, 253-291.

Liu, Y., Xi, D., Li, Z., \& Hong,Y. 2015 A New Methodology for Pixel-Quantitative Precipitation Nowcasting using a Pyramid Lucas Kanade optical flow Approach. Journal of Hydrology 529: 354-364.

Okamoto, K., Nishio, S., Saga, T., \& Kobayashi, T. 2000 Standard images for particle-image velocimetry. Measurement Science Technology 11, 685-691. 
Pust, Oliver 2000 PIV: Direct Cross-Correlation compared with FFT-based Cross-Correlation. In: Proceedings of the $10^{\text {th }}$ International Symposium on Applications of Laser Techniques to Fluid Mechanics. Lisbon, Portugal.

Quènot, G. M., Pakleza, J., \& Kowalewski, T. A. 1998 Particle image velocimetry with optical flow. Experiments in Fluids 25, 177-189.

Raffel, M., Willert, C. E., Wereley, S. T., \& Kompenhans, J. 2007 Particle Image Velocimetry: A Practical Guide, chapter 3-5. Springer.

Wang, B., Cai, Z., Shen, L., \& Liu, T. 2014 An analysis of physics-based optical flow. Journal of Computational and Applied Mathematics 276, 62-80.

Yang, Z., Yu, H., Huang, G., Ludwig, B. 2016 Improved optical flow method for blood velocimetry. Medical Physics, (Under review). 